\title{
Low-mass X-ray binaries from black hole retaining globular clusters
}

\author{
Matthew Giesler, ${ }^{\star}$ Drew Clausen and Christian D. Ott \\ TAPIR, Walter Burke Institute for Theoretical Physics, California Institute of Technology, Pasadena, CA 91125, USA
}

Accepted 2018 March 8. Received 2018 January 30; in original form 2017 August 19

\begin{abstract}
Recent studies suggest that globular clusters (GCs) may retain a substantial population of stellar-mass black holes (BHs), in contrast to the long-held belief of a few to zero BHs. We model the population of BH low-mass X-ray binaries (BH-LMXBs), an ideal observable proxy for elusive single BHs, produced from a representative group of Milky Way GCs with variable $\mathrm{BH}$ populations. We simulate the formation of $\mathrm{BH}$ binaries in GCs through exchange interactions between binary and single stars in the company of tens to hundreds of BHs. Additionally, we consider the impact of the BH population on the rate of compact binaries undergoing gravitational wave driven mergers. The characteristics of the BH-LMXB population and binary properties are sensitive to the GCs structural parameters as well as its unobservable $\mathrm{BH}$ population. We find that GCs retaining $\sim 1000 \mathrm{BHs}$ produce a galactic population of $\sim 150$ ejected BH-LMXBs, whereas GCs retaining only $\sim 20$ BHs produce zero ejected BH-LMXBs. Moreover, we explore the possibility that some of the presently known BH-LMXBs might have originated in GCs and identify five candidate systems.
\end{abstract}

Key words: gravitational waves - stars: black holes - stars: low-mass - globular clusters: general-X-rays: binaries.

\section{INTRODUCTION}

The fate of the population of stellar-mass black holes $(\mathrm{BH})$ in globular clusters (GCs) is still widely uncertain. It is expected that tens to hundreds and possibly thousands of BHs are formed in GCs, of which some fraction might be ejected early due to a kick at formation (Belczynski et al. 2006). In the standard GC evolution picture, the remainder of the BHs should rapidly sink to the core due to mass segregation. There they are subject to a high rate of dynamical interactions that are likely to eject the BHs as singles or in binaries. It was long accepted that this process would lead to repeated ejections from the GC leaving a few to zero BHs (e.g. Kulkarni, Hut \& McMillan 1993; Sigurdsson \& Hernquist 1993). Historically, this was supported by the lack of observational evidence for a $\mathrm{BH}$ in a GC; however, BHs are difficult to observe unless they are actively accreting from a stellar companion.

In order to explore the population of BHs within and outside of GCs, BH low-mass X-ray binaries (BH-LMXBs) can serve as an ideal proxy. In an evolved cluster, a main-sequence (MS) star will necessarily be less than the MS turn-off mass, yielding an abundance of potential low-mass companions. This, coupled with a high rate of encounters due to the high-density environment of GCs, makes GCs ideal BH-LMXB factories. However, this assumes that a significant number of BHs are retained by GCs and that the BHs avoid segregating completely from the lower-mass stars.
The discovery of two BH-LMXB systems in the Milky Way GC M22 (Strader et al. 2012) has led to a renewed interest in GC $\mathrm{BH}$ retention. This observation coupled with an estimate for the fraction of the $\mathrm{BH}$ population expected to be in accreting binaries (Ivanova et al. 2010) suggests that M22 may contain 5-100 BHs (Strader et al. 2012). Additionally, Di Stefano et al. (2002) suggested a number of high-luminosity LMXBs residing in M31 GCs may harbour BH primaries, which was confirmed by Barnard et al. (2011). Recent theoretical studies, including some detailed $N$-body simulations (e.g. Aarseth 2012; Wang, Jia \& Li 2016), support the idea that GCs are capable of retaining from a few to hundreds of BHs (e.g. Breen \& Heggie 2013; Morscher et al. 2013; Sippel \& Hurley 2013; Rodriguez et al. 2016b).

There is an increasing number of BH-LMXB candidates identified in the Milky Way galaxy. BlackCAT (Corral-Santana et al. 2016), a catalogue of BH-LMXBs, has to date identified 59 candidate Milky Way BH-LMXBs. An LMXB is identified as a candidate BH-LMXB if the X-ray spectrum rules out a neutron star (NS) as the compact accretor (McClintock \& Remillard 2006). Of the 59 candidate BH-LMXBs in BlackCAT, 22 are currently considered to be 'confirmed' BH-LMXBs. A BH-LMXB labelled as 'confirmed' has a dynamical measurement of the primary mass or mass function $f\left(M_{\mathrm{BH}}\right)$ (see, e.g. Casares \& Jonker 2014).

Roughly one-fifth of the observed BH-LMXBs reside at an absolute distance $|z|$ perpendicular to the Galactic plane greater than $1 \mathrm{kpc}$ (e.g. Jonker \& Nelemans 2004; Corral-Santana et al. 2016). The distribution of the candidate and confirmed BH-LMXBs within the Milky Way gives rise to the idea that BHs might be subject 
to high-velocity kicks at formation (e.g. Gualandris et al. 2005; Fragos et al. 2009; Repetto, Davies \& Sigurdsson 2012; Repetto $\&$ Nelemans 2015). In some cases, the velocity needed for the binary to reach large $|z|$ exceeds the contribution from a Blaauw kick (Blaauw 1961). This is the velocity imparted to a binary in the case of sudden mass-loss, i.e. in the BH progenitor's supernova explosion. The exceptional high-velocity BH-LMXB cases have led to the idea of high-velocity formation kicks, also known as 'natal' kicks, where the binary receives a large kick through an asymmetric explosion launched prior to BH formation (Janka 2013; Janka 2017). Due to the long-held assumption that GCs maintain a nearzero population of $\mathrm{BHs}$, the possibility that some of these systems originated in GCs has been largely ignored. BH-LMXBs sourced by $\mathrm{BH}$-retaining GCs might help to explain some of the peculiar properties of the observed Milky Way BH-LMXB population. Although GCs are not likely to describe the entire population of BH-LMXBs, the halo-orbits of GCs in the Milky Way make GCs ideal candidate sources for the high- $|z|$ systems. In light of the recent studies that suggest GCs might harbour a large number of BHs, we revisit in this paper the possibility of GCs as a potential origination point for a subset of the observed BH-LMXB systems.

Although we are primarily concerned with the Galactic population of BH-LMXBs evolving from initially non-mass-transferring binaries ejected from GCs, BH-LMXBs can form within GCs through more exotic channels. These formation channels include mass-transfer following directly from a physical collision or tripleinduced mass transfer coupled with exchange encounters or physical collisions (Ivanova et al. 2010). The more recent work of Ivanova et al. (2017) proposes a new BH population-dependent channel for the production of $\mathrm{BH}-\mathrm{LMXBs}$ within GCs by means of grazing tidal encounters between a $\mathrm{BH}$ and a sub-giant.

In addition to using $\mathrm{BH}-\mathrm{LMXBs}$ as probes of $\mathrm{BH}$ retention in $\mathrm{GCs}$, the $\mathrm{BH}-\mathrm{BH}$ merger rates might also serve to place some constraints on $\mathrm{GC} \mathrm{BH}$ retention. The recent success in observing merging $\mathrm{BH}-\mathrm{BH}$ binaries by advanced LIGO (aLIGO) makes this a realistic possibility (Abbott et al. 2016a,b,c). Furthermore, binary $\mathrm{BH}$ mergers occurring in GCs may be characteristically eccentric due to dynamical formation channels. Although these eccentric systems are likely to have circularized by the time they are visible in the aLIGO frequency band, the eccentricity is potentially detectable at lower frequencies. The addition of a space-based gravitational wave observatory (e.g. LISA) in the future, designed for sensitivity at lower frequencies, further improves the prospect of using $\mathrm{BH}-\mathrm{BH}$ mergers to probe GC dynamics.

In this study, we explicitly evolve 'test' binaries in a fixed cluster background subject to dynamical friction and single-binary interactions. Additionally, we include an updated prescription for allowing single BHs to exchange into existing binaries. The GCs are chosen to represent a realistic subset of Milky Way GCs with varying BH populations in order to investigate the effects of $\mathrm{BH}$ retention in clusters. Each GC background is described by an isotropic multimass King model. We produce a large number of realizations for each set of initial parameters to obtain statistical distributions of the number of ejected binaries and their relevant properties. Using the statistics from the GC simulations, we then perform Monte Carlo simulations to obtain a population of BH-LMXBs produced by GCs. The GCs and the ejected binaries are evolved in time through the Milky Way potential while simultaneously accounting for the stellar evolution of the ejected binaries. The resulting mass-transferring systems make up a previously unexplored Galactic population of BH-LMXBs from GCs. We investigate the distribution and properties of the resulting population and its dependence on $\mathrm{BH}$ retention in GCs. Specifically, we find that in the case of minimal $\mathrm{BH}$ retention $\left(N_{\mathrm{BH}}=20\right)$ no observable BH-LMXBs are produced, while the $N_{\mathrm{BH}}=200$ and $N_{\mathrm{BH}}=1000$ cases, respectively, yield Galactic populations of $25_{-6}^{+10}$ and $156_{-24}^{+26}$ BH-LMXBs. Furthermore, we use the resulting population to determine the most likely candidates for a $\mathrm{GC}$ origin in the population of observed Milky Way $\mathrm{BH}$ LMXBs: the five systems that are compatible with our simulated population of BH-LMXBs from GCs are MAXI J1659-152, SWIFT J1357.2-0933, SWIFT J1753.5-0127, XTE J1118+480, and GRO $\mathrm{J} 0422+32$. One caveat is that four out of five of these systems are still lacking measurements of the companion metallicity. Due to the low-metallicity environments of GCs, a measurement finding a metallicity significantly larger than typical GC metallicities would be a strong piece of evidence ruling out a GC origin for the system under consideration. The fifth system, XTE J1118+480, has had its metallicity measured twice. However, the findings of Frontera et al. (2001) and González Hernández et al. (2006) currently provide conflicting claims regarding the companion metallicity. Future measurements will be necessary to increase support for a GC origin theory, but if we can confidently attribute a BH-LMXB to a GC, this would provide strong evidence for significant $\mathrm{BH}$ retention in GCs.

The remainder of this paper is structured as follows. In Section 2, we describe our model for the GCs and the evolution of a test-binary in a static cluster background. In Section 3, we lay out how we generate the present-day BH-LMXB population from our simulations of Milky Way GCs. In Section 4, we review the properties of the ejected $\mathrm{BH}$ binaries along with the distribution and properties of the present-day BH-LMXBs from GCs. Additionally, we explore the effects of $\mathrm{BH}$ retention on the $\mathrm{BH}-\mathrm{BH}$ merger rate in GCs. We conclude the section by comparing our results with observations and previous work. Finally, in Section 5, we provide concluding remarks.

\section{METHODS}

GCs typically contain $\sim 10^{5}-10^{6}$ stars, which makes them accessible to modern $N$-body simulations (e.g. Zonoozi et al. 2011; Wang et al. 2016) that can track GC evolution. However, full $N$-body cluster evolution simulations are still very computationally expensive, making this method poorly suited for studying many realizations of different GCs necessary for building statistics on the evolution of BH binaries inside clusters. Fokker-Planck methods are more approximate and describe GCs with a phase-space distribution function for its constituent stars that evolves via the Fokker-Planck equation, a Boltzmann equation with a small local collision term that modifies only velocities (see e.g. Spitzer 1987). The FokkerPlanck equation can be numerically integrated directly (e.g. Cohn 1979; Chernoff \& Weinberg 1990), or more commonly, integrated with Monte Carlo methods [see e.g. Hénon (1971), Spitzer \& Hart (1971), and Rodriguez et al. (2016b) for a comparison between $\mathrm{N}$-body and the Monte Carlo approaches]. However, here we are concerned with the evolution of BH binaries in GCs and not with the GC evolution itself. Hence, we adopt the approach of modelling the evolution of binaries in a fixed cluster background, pioneered in the early 1990s [see e.g. Hut et al. (1992), Davies \& Benz (1995), Davies (1995), Sigurdsson \& Phinney (1995), and Benacquista \& Downing (2013) for an overview of the theoretical models of GCs and the dynamics occurring within]. We approximate the collision term in the Fokker-Planck equation analytically to model the effects of distant encounters as the binary evolves through the GC. Near encounters are accounted for by explicitly integrating the 
three-body equations of motion. We build up statistics by carrying out simulations of many random realizations of binaries for a given GC background model. In the following sections, we describe our method in detail.

\subsection{Model}

Our model, most closely based on Sigurdsson \& Phinney (1995), incorporates a number of assumptions that simplify the simulations and allow us to perform $\sim 10^{4}$ realizations for a given cluster model with relatively minimal computational needs. The three key assumptions are (i) GCs are well described by a 'lowered Maxwellian' distribution function, (ii) the gravitational potential and distribution functions are stationary, and (iii) the effect of distant interactions is well described by the leading order terms in the Fokker-Planck equation. The 'lowered Maxwellian' distribution function, which eliminates the tail of the Maxwellian velocity distribution, introduces a maximum energy for stars within the cluster to remain bound. This maximum energy $\phi\left(r_{\mathrm{t}}\right)$ implies a finite mass and a maximum radius $r_{\mathrm{t}}$, commonly referred to as the 'tidal' radius, as stars beyond this distance are pulled from the cluster by the Galactic tidal field. Models based on a 'lowered Maxwellian', commonly referred to as King models, readily describe many observed clusters (Peterson \& King 1975; Bahcall \& Hausman 1977; Spitzer 1987).

We evolve a single 'test binary', initialized according to Section 2.2.5, in a static cluster background described by an isotropic multimass King model (King 1966) defined by single particle distribution functions $f_{\alpha}\left(\boldsymbol{r}, \boldsymbol{v}, m_{\alpha}\right)$ for a discrete set of mass groups. Here, $\boldsymbol{r}$ and $\boldsymbol{v}$ are the radius and velocity in the cluster centreof-mass frame and $m_{\alpha}$ is the representative mass of group $\alpha$. The distribution function for a given mass group is given by

$f_{\alpha}(\varepsilon)= \begin{cases}\frac{n_{0_{\alpha}}}{\left(2 \pi \sigma_{\alpha}^{2}\right)^{3 / 2}}\left(\mathrm{e}^{-\varepsilon / \sigma_{\alpha}^{2}}-1\right) & \varepsilon<0 \\ 0 & \varepsilon \geq 0 .\end{cases}$

Here, $\varepsilon$ is the energy per unit mass, $\varepsilon=v^{2} / 2-\Psi(r)$, and $\Psi(r) \equiv \phi\left(r_{\mathrm{t}}\right)-\phi(r)$ is the gravitational potential relative to that at the tidal radius $r_{\mathrm{t}}$. Additionally, $\sigma_{\alpha}$ is the group's velocity dispersion at the core of the cluster and $n_{0_{\alpha}}$ is a normalization factor. For an isotropic cluster, the velocity dispersion reduces to the one-dimensional mean-square velocity, such that $3 \sigma_{\alpha}^{2}=\bar{v}_{\alpha}^{2}$. The normalization factor in its full form is

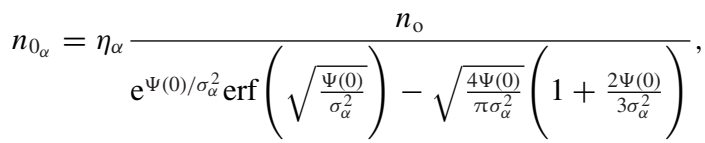

where $\eta_{\alpha}=N_{\alpha} / N$ is the number fraction for mass group $\alpha$ and $n_{\mathrm{o}}=n(0)$ is the central density.

The free structural parameters necessary to specify a model cluster, with specified mass groups, are the mean core velocity dispersion $\bar{\sigma}$, the core number density $n_{\mathrm{o}}$, and the potential depth, which is specified by the dimensionless King parameter $W_{\mathrm{o}}=\Psi(0) / \bar{\sigma}^{2}$. The remaining structural parameters, which are fully determined by the free parameters, are total mass $M_{\mathrm{c}}$, core radius $r_{\mathrm{c}}$, tidal radius $r_{\mathrm{t}}$, and concentration $c=\log _{10}\left(r_{\mathrm{t}} / r_{\mathrm{c}}\right)$. The core radius $r_{\mathrm{c}}$ is defined as the radius at which the surface brightness has dropped to half the value at the core.

For a given set of masses with corresponding distribution functions, the cluster satisfies Poisson's equation for the relative poten- tial $\nabla^{2} \Psi(r)=-4 \pi G \sum_{\alpha} \rho_{\alpha}$. Here, $\rho_{\alpha}=m_{\alpha} n_{\alpha}$, where $n_{\alpha}$ is the number density of mass group $\alpha$ given by

$n_{\alpha}=\int_{0}^{v\left(r_{\mathrm{t}}\right)} f_{\alpha}\left(\boldsymbol{r}, \boldsymbol{v}, m_{\alpha}\right) 4 \pi v^{2} \mathrm{~d} v$

The upper limit of the integral is the maximum allowed velocity $v\left(r_{\mathrm{t}}\right)=\sqrt{2 \Psi\left(r_{\mathrm{t}}\right)}$, i.e. the escape velocity. The object masses $m_{\alpha}$ and number fraction $\eta_{0_{\alpha}}$ are determined by the evolved mass function (EMF), discussed in Section 2.2.1. We generate a model cluster that satisfies Poisson's equation for the specified masses and number fractions in an iterative fashion. We begin by integrating Poisson's equation out to a radius $r_{\mathrm{t}}$, implicitly determined by $\Psi\left(r_{\mathrm{t}}\right)=0$, with boundary conditions $\Psi(0)=W_{\mathrm{o}}$ and $\nabla \Psi(0)=0$, and take $\eta_{\alpha}=\eta_{0_{\alpha}}$ as our initial guess. The actual number fraction of each mass group, $\eta_{\alpha}=N_{\alpha} / N$, is then calculated using

$N_{\alpha}=\int_{0}^{r_{\mathrm{t}}} n_{\alpha}(r) 4 \pi r^{2} \mathrm{~d} r$

along with $N=\sum_{\alpha} N_{\alpha}$. We then update our guess to $\eta_{\alpha}=\left(\eta_{\alpha_{\text {new }}}+\right.$ $\left.\eta_{\alpha_{\text {old }}}\right) / 2$, where $\eta_{\alpha_{\text {new }}} \rightarrow \eta_{\alpha_{\text {old }}} \times\left(\eta_{0_{\alpha}} / \eta_{\alpha}\right)$. We repeat the above steps until $\left(\eta_{0_{\alpha}}-\eta_{\alpha}\right) / \eta_{0_{\alpha}}<\delta$ is satisfied for all mass groups, where we have made the somewhat arbitrary choice of $\delta=6.25 \times 10^{-3}$ for our convergence threshold. This iterative procedure determines the normalization constant $n_{0_{\alpha}}$ and $r_{\mathrm{t}}$. Once $r_{\mathrm{t}}$ is found, the concentration $c=\log _{10}\left(r_{\mathrm{t}} / r_{\mathrm{c}}\right)$ is determined and the total mass of the cluster $M_{\mathrm{c}}$ is obtained from

$-\nabla \Psi\left(r_{\mathrm{t}}\right)=\frac{G M}{r_{\mathrm{t}}^{2}}$.

The evolution of our 'test binary' in the cluster background is affected by long-range and short-range interactions, which modify the magnitude and direction of the binary's velocity. The shortrange encounters are accounted for by fully resolving the threebody interactions, detailed in Section 2.3.4. We account for the velocity fluctuations due to long-range interactions with 'field stars', distant cluster stars, through the diffusion coefficients $D\left(\Delta v_{i}\right)$ and $D\left(\Delta v_{i} \Delta v_{j}\right)$ in the Fokker-Planck equation,

$$
\begin{aligned}
\frac{D f}{D t} & =\left(\frac{\partial f}{\partial t}\right)_{\mathrm{enc}} \\
& =\sum_{i, j}\left\{-\frac{\partial}{\partial v_{i}}\left(D\left(\Delta v_{i}\right) f\right)+\frac{1}{2} \frac{\partial^{2}}{\partial v_{i} \partial v_{j}}\left(D\left(\Delta v_{i} \Delta v_{j}\right) f\right)\right\}
\end{aligned}
$$

In this context, a diffusion coefficient $D(X)$ for a variable $X$ corresponds to the average change in $X$ per unit time. Here, we focus on velocity changes per unit time as experienced by the binary due to interactions with the 'field stars'. The form of the coefficients can be derived, for a simple case, by first considering the change in velocity of a mass $m_{1}$, initially at rest, due to an encounter with a second mass $m_{2}$ at a relative velocity $v$ with impact parameter $p$,

$(\Delta v)^{2}=\frac{4 m_{1}^{2}}{\left(m_{1}+m_{2}\right)^{2}} \frac{v^{2}}{\left(1+\left(\frac{p}{p_{0}}\right)^{2}\right)}$,

where $p_{\mathrm{o}} \equiv G\left(m_{1}+m_{2}\right) / v^{2}$ is a reference impact parameter that causes a deflection of $\pi / 2$, consistent with close encounters (e.g. Spitzer 1987). The average rate of change of the quantity in equation (7), per unit time, due to encounters is then obtained by integrating over the possible impact parameters for a given density of field stars $n$,

$D\left(\Delta v^{2}\right)=2 \pi \int_{0}^{p_{\max }} \Delta v^{2} p n v \mathrm{~d} p$ 
up to a maximum allowable impact parameter $p_{\max }$. The maximum impact parameter is required to suppress the divergence of the integral and essentially determines the maximum distance of long-range encounters that contribute to the velocity perturbations. This maximum value, $p_{\max }$, is not explicitly specified, but finds its way into the coefficient calculations through the so-called Coulomb logarithm, $\ln \Lambda \equiv \ln \left(p_{\max } / p_{\mathrm{o}}\right)$, which appears as a result of the integration.

We work out the details for the case of an isotropic velocity dispersion with a density of field stars given by equation (3) and restate the relevant coefficients we use in our model (cf. Binney \& Tremaine 2008). These coefficients, which describe the average rate of change in the velocity of the binary due to long-range encounters, are used to update the velocity of the binary at each time-step. The implementation is described further in Section 2.3. A detailed derivation and a more general form of the coefficients can be found in Spitzer (1987).

By choosing a coordinate system in which one axis is aligned with the velocity of the binary, we can decompose $D\left(\Delta v_{i}\right)$ into a coefficient parallel to the binary's velocity $D\left(\Delta v_{\|}\right)$and two mutually orthogonal coefficients perpendicular to the velocity, $D\left(\Delta v_{\perp}\right)_{1}$ and $D\left(\Delta v_{\perp}\right)_{2}$. In an isotropic cluster, there is no preferred direction with regard to the two perpendicular components, so the contributions from $D\left(\Delta v_{\perp}\right)_{1}$ and $D\left(\Delta v_{\perp}\right)_{2}$ tend to cancel each other out; however, their squares, $D\left(\Delta v_{\perp}^{2}\right)_{1}$ and $D\left(\Delta v_{\perp}^{2}\right)_{2}$, on the other hand, do not and are non-vanishing. Additionally, we include a quadratic term for the parallel component $D\left(\Delta v_{\|}^{2}\right)$ and in consideration of the symmetry we retain only the sum of the perpendicular components $D\left(\Delta v_{\perp}^{2}\right)=D\left(\Delta v_{\perp}^{2}\right)_{1}+D\left(\Delta v_{\perp}^{2}\right)_{2}$.

The diffusion coefficient $D\left(\Delta v_{\|}\right)$parallel to the binary's motion is by analogy often referred to as the coefficient of dynamical friction as it opposes the binary's direction of motion,

$D\left(\Delta v_{\|}\right)=-\sum_{\alpha} \gamma_{\alpha}\left(1+\frac{m_{\mathrm{b}}}{m_{\alpha}}\right) \int_{0}^{v}\left(\frac{v_{\alpha}}{v}\right)^{2} f_{\alpha}\left(v_{\alpha}\right) \mathrm{d} v_{\alpha}$.

Here, $m_{\mathrm{b}}$ is the mass of the binary and $\gamma_{\alpha} \equiv\left(4 \pi G m_{\alpha}\right)^{2} \ln \Lambda$, where we have chosen to set $\ln \Lambda=10$, a value typical for GCs (Spitzer 1987). The two remaining coefficients,

$$
D\left(\Delta v_{\|}^{2}\right)=\sum_{\alpha} \frac{2}{3} v \gamma_{\alpha}\left\{\int_{0}^{v}\left(\frac{v_{\alpha}}{v}\right)^{4}+\int_{v}^{\infty}\left(\frac{v_{\alpha}}{v}\right)\right\} f_{\alpha}\left(v_{\alpha}\right) \mathrm{d} v_{\alpha}
$$

and

$$
\begin{aligned}
& D\left(\Delta v_{\perp}^{2}\right)=\sum_{\alpha} \frac{2}{3} v \gamma_{\alpha} \\
& \times\left\{\int_{0}^{v}\left[3\left(\frac{v_{\alpha}}{v}\right)^{2}-\left(\frac{v_{\alpha}}{v}\right)^{4}\right]+2 \int_{v}^{\infty}\left(\frac{v_{\alpha}}{v}\right)\right\} f_{\alpha}\left(v_{\alpha}\right) \mathrm{d} v_{\alpha},
\end{aligned}
$$

are strictly positive. These coefficients are responsible for the stochastic perturbations to the parallel and perpendicular components of the velocity, which take the binary on a random walk through velocity space and compete with the slowing due to dynamical friction. We implement these 'random kicks' as discrete changes to the binary's velocity by sampling from a normalized distribution of the velocity perturbations, described in Section 2.3.

\subsection{Initial conditions}

\subsubsection{Evolved mass function}

We obtain an initial distribution of masses in the range $0.08 \mathrm{M}_{\odot}<m<120 \mathrm{M}_{\odot}$ from the broken-power-law initial mass function (IMF)

$\xi(m) \propto \begin{cases}m^{-1.3} m_{x}^{0.3-x_{*}} & m<m_{x} \\ m^{-1.0-x_{*}} & m \geq m_{x},\end{cases}$

with $x_{*}=1.35$ and $m_{x}=0.55 \mathrm{M}_{\odot}$ chosen to incorporate a Salpeter IMF (Salpeter 1955) for masses above $m_{x}$ and a Kroupa 'correction' (Kroupa 2001) to masses below $m_{x}$ along with a normalization factor for continuity. Stars with masses below the MS turn-off, which we set to $m_{\mathrm{to}}=0.85 \mathrm{M}_{\odot}$ (Meylan \& Heggie 1997), are assumed not to evolve significantly on the time-scale of the simulations, while masses above $m_{\text {to }}$ are assumed to be completely evolved according to a specified EMF. The evolved mass $m_{\mathrm{e}}$ is determined by the EMF:

$m_{\mathrm{e}}= \begin{cases}m_{\mathrm{MS}}=m & 0.08 \mathrm{M}_{\odot}<m \leq m_{\mathrm{to}} \\ m_{\mathrm{WD}}=0.45+0.12(m-1) & m_{\mathrm{to}}<m<8 \mathrm{M}_{\odot} \\ m_{\mathrm{NS}}=1.4 & 8 \mathrm{M}_{\odot} \leq m<20 \mathrm{M}_{\odot} \\ m_{\mathrm{BH}}=m_{\mathrm{BH}}\left(m, f_{\mathrm{s}_{\mathrm{BH}}}\right) & 20 \mathrm{M}_{\odot}<m<120 \mathrm{M}_{\odot},\end{cases}$

where the mass subscripts label the object type and refer to main sequence (MS), white dwarf (WD), neutron star (NS), and black hole $(\mathrm{BH})$. We occasionally refer to the set of MS and WD objects as the non-compact (NC) population. The MS stars below the turnoff mass are set to their zero-age main-sequence (ZAMS) mass, the WD stars are a linear function of their ZAMS mass (Catalán et al. 2008), and NS are simply set to $1.4 \mathrm{M}_{\odot}$. Following the work of Sana et al. (2012), the BHs are assumed to have formed from two possible channels: stars with companions that significantly affect the evolution of the star and those stars that are 'effectively single.' Effectively single is used to describe stars that evolve in isolation as well as those stars that evolve in wide binaries with minimal interaction. Sana et al. (2012) estimate that $\sim 70$ per cent of massive stars will have their final state impacted by a companion, which motivates setting $f_{\mathrm{s}_{\mathrm{BH}}}=0.3$ for the fraction of $\mathrm{BHs}$ that formed in isolation. This fraction of BHs that evolve from 'effectively single' stars are void of the complexities of binary stellar evolution and are assumed to lose a significant fraction of their hydrogen shells to stellar winds before collapsing to a $\mathrm{BH}$. For the low metallicities typical of GCs, we approximate the mass-loss, as $\sim 10$ per cent of the initial mass and set $m_{\mathrm{e}}=0.9 \mathrm{~m}$. The remaining 70 per cent of BHs formed will have evolved with a companion and likely passed through a common envelope phase, stripping the stars down to their helium (He) cores (Sana et al. 2012; de Mink et al. 2014). Using MESA (Paxton et al. 2011) to evolve masses in the range $20 \mathrm{M}_{\odot}<m<120 \mathrm{M}_{\odot}$, we obtained the He core mass as a function of the ZAMS mass in order to determine the remnant mass for the remaining $\left(1-f_{\mathrm{S}_{\mathrm{BH}}}\right)$ fraction of $\mathrm{BHs}$ :

$m_{\mathrm{e}}=m_{\mathrm{He}}=0.2312\left(m_{\mathrm{ZAMS}}\right)^{1.1797} \mathrm{M}_{\odot}$.

The stellar evolution performed using MESA version 6794, follows the procedure laid out in Morozova et al. (2015).

Fig. 1 displays the resulting $\mathrm{He}$ core mass as a function of the ZAMS mass from the MESA runs with metallicity $Z=5 \times 10^{-4}$, along with the power-law fit of equation (14). This metallicity 


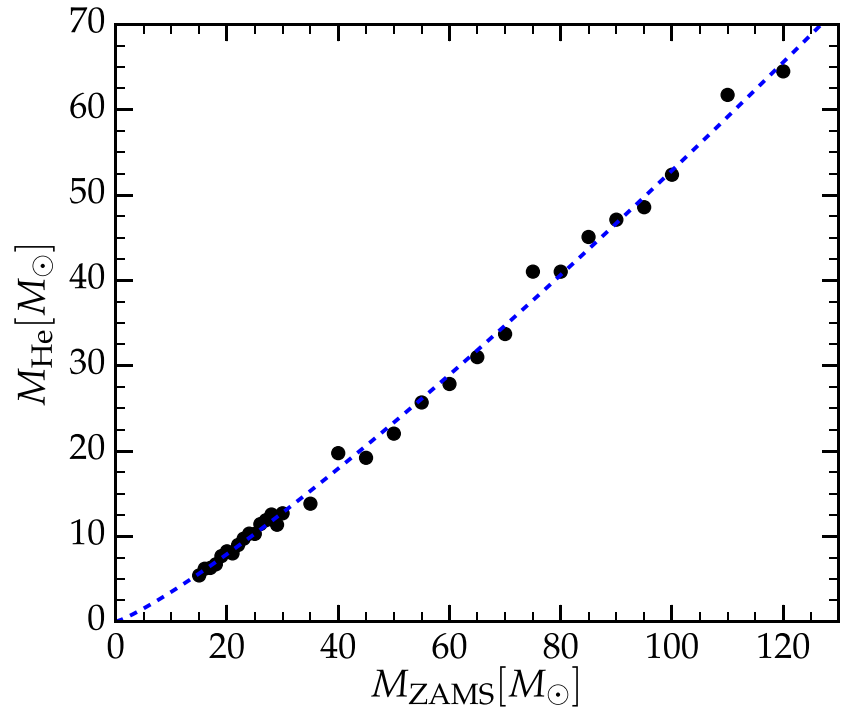

Figure 1. The He core mass (marked by circles) as a function of ZAMS mass from the MESA (Paxton et al. 2011) runs, along with the fit (blue, dashed line) given by equation (14). For the $\sim 70$ per cent of BHs formed in binaries, we approximate the remnant $\mathrm{BH}$ mass with the $\mathrm{He}$ core mass of the progenitor. The remnant mass for the remaining $\sim 30$ per cent of BHs is approximated by $0.9 M_{\text {ZAMS }}$, which accounts for the hydrogen mass lost to stellar winds at low metallicity.

corresponds to the higher peak in the bimodal, GC metallicity distribution (Harris 1996). In order to properly account for the range of metallicities in our sample of clusters (see Table 2), we repeat the same process with $Z=5 \times 10^{-3}$, corresponding to the secondary peak in the GC metallicity distribution. However, as this order of magnitude difference in metallicity produces He core masses differing by $\lesssim 10$ per cent, we rely on equation (14) as a good approximation for the remnant masses in all modelled clusters.

In addition to specifying the evolved masses, it is also necessary to specify the number of NS and BH objects retained by the cluster in its static state. We specify the retained population of compact objects, comprised of NSs and BHs, through the retention fractions $f_{\mathrm{r}_{\mathrm{NS}}}$ and $f_{\mathrm{r}_{\mathrm{BH}}}$, respectively. This is necessary since we are modelling the cluster in its evolved state, a time at which many of the NS and BHs formed within the cluster have already been ejected due to formation kicks. Studies of the proper motion of pulsars suggest that NSs receive kicks in the range of $200-450 \mathrm{~km} \mathrm{~s}^{-1}$ (Lyne \& Lorimer 1994), easily exceeding the typical escape velocity of clusters, which is on the order of tens of $\mathrm{km} \mathrm{s}^{-1}$. However, the observations of pulsars in GCs implies a 'retention problem', since the observed fraction retained is inconsistent with the average natal kick velocities being significantly greater than GC escape velocities. This issue is somewhat reconciled by assuming some NS form in binaries, which dampen the kick and allow the GC to maintain a hold on the NS and companion (Pfahl, Rappaport \& Podsiadlowski 2002). In consideration of these observations, for the case of NSs, we retain a constant fraction, $f_{\mathrm{r}_{\mathrm{NS}}}=0.1$, of those produced by the IMF (Sigurdsson \& Phinney 1995; Pfahl et al. 2002; Ivanova et al. 2008). In the BH case, the distribution of natal kicks is highly uncertain. Rather than take the retention fraction $f_{\mathrm{r}_{\mathrm{BH}}}$ to be a constant across clusters, as in the NS case, we utilize this fraction as a free parameter in our models to control the number of retained BHs in each modelled GC.

Once we have determined the evolved masses from the IMF, the masses are binned into 12 groups. The small number of bins
Table 1. Evolved mass groups for NGC $6121\left(N_{\mathrm{BH}}=200\right)$ with corresponding mass index, the lower boundary bin mass $m_{\min }$, the upper boundary bin mass $m_{\max }$, the average mass of the group $\bar{m}$, the fraction of the total mass in the cluster $f_{\mathrm{m}}$, the number fraction with respect to the total number of objects in the cluster $f_{\mathrm{n}}$, and the fraction of luminous objects in the group $f_{\mathrm{L}}$. For reference, the BH masses occupy the top three mass groups with mean masses of $8.87 \mathrm{M}_{\odot}, 20.48 \mathrm{M}_{\odot}$, and $57.18 \mathrm{M}_{\odot}$.

\begin{tabular}{lcccccc}
\hline $\begin{array}{l}\text { Mass } \\
\text { group }\end{array}$ & $m_{\min }\left(\mathrm{M}_{\odot}\right)$ & $m_{\max }\left(\mathrm{M}_{\odot}\right)$ & $\bar{m}\left(\mathrm{M}_{\odot}\right)$ & $f_{\mathrm{m}}$ & $f_{\mathrm{n}}$ & $f_{\mathrm{L}}$ \\
\hline 0 & 0.08 & 0.200 & 0.12827 & 0.17531 & 0.42853 & 1.0000 \\
1 & 0.20 & 0.350 & 0.26596 & 0.17757 & 0.20933 & 1.0000 \\
2 & 0.35 & 0.450 & 0.40704 & 0.13954 & 0.10748 & 0.7552 \\
3 & 0.45 & 0.600 & 0.51190 & 0.24921 & 0.15264 & 0.5763 \\
4 & 0.60 & 0.700 & 0.64624 & 0.10020 & 0.04861 & 0.7644 \\
5 & 0.70 & 0.850 & 0.76855 & 0.11027 & 0.04499 & 0.8233 \\
6 & 0.85 & 1.000 & 0.91758 & 0.01161 & 0.00397 & 0.0000 \\
7 & 1.00 & 1.200 & 1.08980 & 0.01005 & 0.00289 & 0.0000 \\
8 & 1.20 & 1.500 & 1.29547 & 0.00527 & 0.00128 & 0.0000 \\
9 & 1.50 & 10.00 & 8.87443 & 0.00143 & 0.00005 & 0.0000 \\
10 & 10.0 & 40.00 & 20.4808 & 0.01261 & 0.00019 & 0.0000 \\
11 & 40.0 & 120.0 & 57.1851 & 0.00693 & 0.00004 & 0.0000 \\
\hline & & & & & &
\end{tabular}

allows for a proper representation of the true distribution while keeping the computational costs to a minimum. Poisson's equation is then integrated to determine the final structural parameters as discussed in Section 2.1. For illustrative purposes, the evolved mass distribution for NGC 6121 with 200 retained BHs is given in Table 1. The bins for each mass group, the mean mass in each bin, and the fraction of luminous objects are constant across simulations; however, the mass fraction and number fraction depend on the structure of the cluster and the number of BHs.

\subsubsection{Core density}

As discussed in Section 2.1, one of the free parameters in our model when specifying a cluster's structure is the core number density $n_{\mathrm{o}}$. However, because this parameter is not easily observable, a GC's density is often reported in terms of a central luminosity density $\rho_{\mathrm{L}}$. For each mass group, we determine a central luminous number density $n_{\mathrm{L}_{\alpha}}=f_{\mathrm{L}_{\alpha}} \bar{n}_{\alpha}$, where $f_{L_{\alpha}}$ and $\bar{n}_{\alpha}$ are the fraction of luminous objects and the core density, respectively, of mass group $\alpha$. The central luminosity density is then given by $\rho_{\mathrm{L}}=\sum_{\alpha} L_{\alpha} n_{\mathrm{L}_{\alpha}}$. In order to account for the variability in the mass-luminosity relation with stellar mass, we use a parametrized luminosity for each group of the form $L_{\alpha}=a\left(m_{\alpha}\right)^{b}$, with luminosity coefficients $a=0.23$, $b=2.3$ for $m_{\alpha}<0.43 \mathrm{M}_{\odot}$ and $a=1.0, b=4.0$ for the remaining luminous objects (Duric 2004). To ensure that our clusters appropriately model the Milky Way GCs of interest, we compute $\rho_{\mathrm{L}}$ for each integrated cluster and adjust $n_{\mathrm{o}}$ accordingly to match the observed quantity.

\subsubsection{Binary fraction}

In order to account for the uncertainty in the size of the binary population within a cluster, we allow for a specifiable binary fraction. The fraction of objects that are binaries is

$f_{\mathrm{b}}=\frac{N_{\mathrm{b}}}{N_{\mathrm{s}}+N_{\mathrm{b}}}$,

where $N_{\mathrm{s}}$ and $N_{\mathrm{b}}$ are the number of single objects and binary objects, respectively, and the total number of objects in our model clusters is 
then $N=N_{\mathrm{s}}+2 N_{\mathrm{b}}$. Observations of the binary fraction are limited to the luminous objects within the cluster. Due to this restriction, we take the observed fraction to be determined solely by the MS star binary fraction $f_{\mathrm{obs}}=N_{\mathrm{MS}_{\mathrm{b}}} /\left(N_{\mathrm{MS}_{\mathrm{s}}}+N_{\mathrm{MS}_{\mathrm{b}}}\right)$, where, as above, we respectively refer to $N_{\mathrm{MS}_{\mathrm{s}}}$ and $N_{\mathrm{MS}_{\mathrm{b}}}$ as the number of single and binary MS stars. Using the above definitions along with the fraction of all binaries that are MS-MS binaries, $f_{\mathrm{MS}_{\mathrm{b}}}=N_{\mathrm{MS}_{\mathrm{b}}} / N_{\mathrm{b}}$, and the fraction of objects that are MS stars, $f_{\mathrm{MS}}=N_{\mathrm{MS}} / N$, we convert the observed binary fraction into a uniform total binary fraction for use in our models through the relation

$f_{\mathrm{b}}=\left(\frac{f_{\mathrm{MS}_{\mathrm{b}}}}{f_{\mathrm{MS}}} \frac{\left(f_{\mathrm{obs}}+1\right)}{f_{\mathrm{obs}}}-1\right)^{-1}$.

The number of MS stars, $N_{\mathrm{MS}}$, is determined solely by the IMF and for the simulations in this study we use $f_{\mathrm{MS}_{\mathrm{b}}}=0.23$ (Fregeau, Ivanova \& Rasio 2009). We perform our simulation with $f_{\text {obs }}$ covering a range of values, consistent with theoretical findings, between 5 and 10 per cent (Ivanova et al. 2005), and with observational constraints, between 5 and 20 per cent (Milone et al. 2012). We complete an approximately equal number of simulations for $f_{\text {obs }}$ taking values from the set $\{0.05,0.10,0.20\}$. However, we find that this parameter has a negligible effect on the quantities of interest, so for conciseness, it is not specified in the simulation parameters.

\subsubsection{Modified $\mathrm{BH}$ velocity dispersion}

Recent studies of $\mathrm{BH}$ retention in GCs have shown clusters initially retain between 65 and 90 per cent of the BHs formed in cluster, with the remainder being lost due to formation kicks (Morscher et al. 2015). This is in contrast to the long-standing belief that presentday GCs should be nearly void of BHs. In addition to the increase in retention, Morscher et al. (2015) also found that the retained $\mathrm{BHs}$ remain well mixed with the non-BH population. Follow-up studies support the idea of a large population of BHs that are spread throughout the cluster and are consistent with a recent $10^{6} \mathrm{~N}$-body simulation (Rodriguez et al. 2016b).

In the standard King model, it is common to assume that the mass groups satisfy an equipartition of energy. Specifically,

$m_{\alpha} \sigma_{\alpha}^{2}=\bar{m} \bar{\sigma}^{2}$,

where $m_{\alpha}$ and $\sigma_{\alpha}$ are the mass and velocity dispersion of mass group $\alpha, \bar{m}$ is the mean mass of all objects in the cluster, and $\bar{\sigma}$ is the mean velocity dispersion. However, with this equipartition of kinetic energy amongst all mass groups, the heavier objects then necessarily have lower random velocities compared to the lighter objects and become trapped deep in the gravitational potential at the core of the cluster. With an equipartition of kinetic energy in place, the much more massive BHs densely populate the central region of the cluster, driving the core radius to a small fraction of the tidal radius. This disparity between the core radius and tidal radius leads to concentrations that deviate from observations, limiting the modelled clusters to supporting only a small number of BHs. In order to generate clusters with a significant $\mathrm{BH}$ population that are still representative of observed GCs, motivated by Morscher et al. (2015), we implement a velocity dispersion for the BHs away from energy equipartition. We maintain an equipartition of energy amongst the lower-mass objects and use a modified energy partitioning for the $\mathrm{BHs}$ of the form

$m_{\beta} \sigma_{\beta}^{2}=\frac{\sum m_{\beta}}{\sum m_{\alpha}} \frac{1}{f_{\mathrm{s}}} \bar{m} \bar{\sigma}^{2}$,

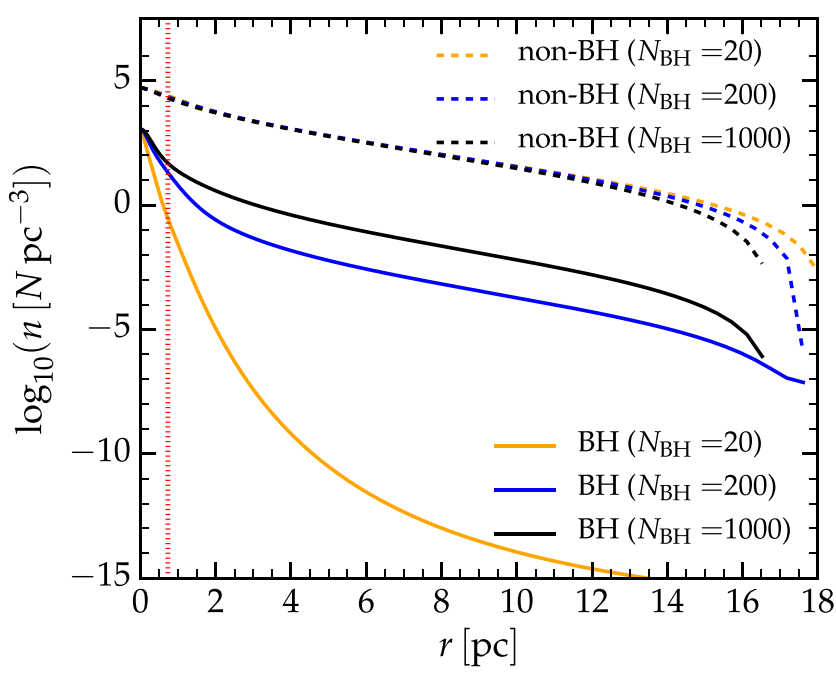

Figure 2. Radial number density profiles for the BH sub-group (solid lines) and the non-BH objects (dashed lines) in NGC 6656 for the three considered values of $N_{\mathrm{BH}}$. The vertical line (red, dashed), at $r_{\mathrm{c}}=0.73 \mathrm{pc}$, marks the core radius for this cluster. The non-BH objects are largely unaffected by the different numbers of $\mathrm{BHs}$ added to the cluster and the necessary modification to the velocity dispersion. For $N_{\mathrm{BH}}=20$, the BHs are concentrated in the core region, whereas to accommodate $N_{\mathrm{BH}} \geq 200$, the modified velocity dispersion spreads the BHs throughout the cluster with a profile similar to that of the non-BH objects.

where the indices $\beta$ and $\alpha$ label the mass groups corresponding to BHs and non-BHs, respectively. Here, $f_{\mathrm{s}}$ is a specifiable scale factor of order unity. The $f_{s}$ parameter is enough to rescale the velocity dispersion for the $\mathrm{BHs}$; however, the factor involving the mass ratio contributes substantially and $f_{s}$ remains of order unity and does not vary wildly across the GCs we consider.

With this modified $\mathrm{BH}$ velocity dispersion in place, we find that we can match the observed structural parameters of a specific cluster

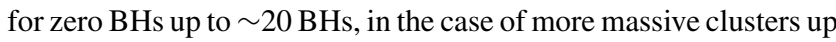
to $\sim 100 \mathrm{BHs}$, and in the most massive clusters up to $\sim 1000 \mathrm{BHs}$. We vary the number of BHs residing in the cluster by adjusting the scale factor $f_{s}$ in equation (18) and the fraction retained, $f_{\mathrm{rBH}}$, introduced in Section 2.2.1. To illustrate the spreading of the BHs, we present in Fig. 2 the radial density profiles for the BHs and the non-BH objects for different populations of retained $\mathrm{BHs}$ in the cluster model representing NGC 6656. In the case of minimal BH retention, the $\mathrm{BH}$ number density falls off quickly outside of the core, which for our model of NGC 6656 is located at $r_{\mathrm{c}}=0.73 \mathrm{pc}$ and is marked by a vertical line in Fig. 2 for reference. However, in the case of many BHs, the modified velocity dispersion extends the number density profile radially, spreading the BHs throughout the cluster, without affecting the central density. The distribution of non- $\mathrm{BH}$ objects is largely unaffected by the change in $\mathrm{BH}$ numbers.

\subsubsection{Binary initialization}

We choose the initial masses for our 'test binary' by randomly sampling from the evolved mass distribution and reject those that do not contain at least one $\mathrm{BH}$. If one of the component masses falls within a mass bin with a non-zero luminous population, we then sample from the luminous mass fraction to determine whether the low-mass object is an MS star or WD. Additionally, if the selected mass is in the turn-off group, $0.63 \mathrm{M}_{\odot} \leq m \leq 0.8 \mathrm{M}_{\odot}$, then the object is chosen to be a giant with probability $P=0.095 f_{\mathrm{L}}$, where $f_{\mathrm{L}}$ is the 
luminous fraction for the turn-off mass group. The probability for giants is adopted from Sigurdsson \& Phinney (1995) and represents the approximate fraction of the cluster age that giants in this mass range survive. Once the masses and object types are established, the $\mathrm{BH}$ radii are set to the Schwarzschild radius $R_{\mathrm{BH}}=2 G M / \mathrm{c}^{2}$, while the stellar radii are determined as described in Sigurdsson \& Phinney (1995). The eccentricity of the binary $e$ is specified by sampling from the probability density function $f(e)=2 e$ (Jeans 1919), commonly referred to as a 'thermal' eccentricity distribution. The semimajor axis $a$ is obtained from a distribution uniform in $\log _{10} a$ in the range $-3 \leq \log _{10}\left(a \mathrm{au}^{-1}\right) \leq 1$. To avoid an immediate merger of the objects in our initial binary, we enforce $a>f_{\text {tid }}\left(R_{1}+R_{2}\right) /(1-e)$, where $R_{i}$ are the radii of each component of the binary and $f_{\text {tid }}=3.1$, by letting $a \rightarrow 2 a$ until this condition is satisfied. The factor $f_{\text {tid }}$ is chosen based on the separation at which tidal effects would induce a merger (Lee \& Ostriker 1986). Once the binary parameters are set, we sample the primary-mass number density profile $n_{\alpha}(r)$ to determine the binary placement within the cluster and obtain a velocity from the primary-mass velocity distribution function at $r$.

\subsection{Evolution of the 'test binary'}

Once we have an appropriate model, which satisfies the structural parameters for a specific cluster and an initial binary, we then evolve this single binary within the cluster background. In addition to the static potential, we include the interaction terms discussed in Section 2.1. To account for dynamical friction, the diffusion coefficient $D\left(\Delta v_{\|}\right)$is added to the potential gradient to create a smooth effective acceleration $\boldsymbol{a}_{\text {eff }}=\nabla \Psi(r)+D\left(\Delta v_{\|}\right)$. This smooth force is integrated using a fourth-order Runge-Kutta integrator, which is discussed in detail in Section 2.3.2. The quadratic scattering terms, or random 'kicks', are implemented by discretely updating the corresponding velocity components at each time-step $\Delta t$. As discussed in Section 2.1, the diffusion coefficient for $\Delta v^{2}$, of the form $D\left(\Delta v^{2}\right)$, represents the change in this quantity per unit time, i.e. $\Delta v^{2} / \Delta t$. We update the velocity at each time-step by sampling from the normal distribution of kicks through

$$
\begin{aligned}
\Delta v_{\|} & =X \sqrt{D\left(\Delta v_{\|}^{2}\right) \Delta t}, \\
\Delta v_{\perp_{1}} & =Y \sqrt{\frac{1}{2} D\left(\Delta v_{\perp}^{2}\right) \Delta t}, \\
\Delta v_{\perp_{2}} & =Y \sqrt{\frac{1}{2} D\left(\Delta v_{\perp}^{2}\right) \Delta t},
\end{aligned}
$$

where $X$ and $Y$ are random numbers with mean values of zero and standard deviations of one.

At each time-step, we also consider the evolution of the binary's semimajor axis $a$ and its eccentricity $e$ due to gravitational wave (GW) emission. If the BH is in a binary with another compact object - which includes BHs, NSs, and WDs - then we implement the evolution of $a$ and $e$ according to the gravitational radiation formalism of Peters (1964). In these cases, we also calculate the time until coalescence $t_{\mathrm{d}}$ due to the decay of $a$, and if this will occur within the current time-step, $t_{\mathrm{d}}<\Delta t$, we consider this a GW merger. If the merger is of a BH-BH or BH-NS binary, we add a recoil velocity, or 'kick', based on the fits to numerical relativity simulations given by Campanelli et al. (2007) with initial spin magnitudes and orientations assigned as in Clausen, Sigurdsson \& Chernoff (2013).

\subsubsection{Short-range encounters}

As the binary moves throughout the cluster, at each time-step, we check for the possibility of a short-range encounter with a single star. Since the effects of long-range interactions are accounted for by the diffusion coefficients (Section 2.1), here we focus on capturing the effects due to strong three-body interactions with much smaller impact parameters. We limit the range of encounters to include only those three-body interactions that result in a resonance, exchange, ionization, or the occasional flyby. We accomplish this by choosing the maximum impact parameter to be

$p=a[B+C(1+e)]$,

where we have set $B=4$ and $C=0.6$ following Hut $\&$ Bahcall (1983). The choice of these coefficients is intended to limit the number of weak encounters that have minimal impact on the binary, as these still require full resolution of the encounter, which is one of the more computationally intensive tasks during evolution. However, the coefficients only provide an approximate contour in the space of initial conditions, hence the occasionally flyby. The cross-section for an encounter to take place between the binary and a star of mass $m_{\alpha}$ with velocity $\boldsymbol{v}_{\alpha}$ is

$\sigma\left(\boldsymbol{v}, \boldsymbol{v}_{\alpha}\right)=\pi p^{2}+\frac{2 \pi G\left(m_{\mathrm{b}}+m_{\alpha}\right) p}{\left|\boldsymbol{v}-\boldsymbol{v}_{\alpha}\right|^{2}}$,

(see e.g. Spitzer 1987). We then calculate the expected encounter rate between the binary and each mass group

$\Gamma(r, v, \alpha)=\int \sigma\left(\boldsymbol{v}, \boldsymbol{v}_{\alpha}\right)\left|\boldsymbol{v}-\boldsymbol{v}_{\alpha}\right| f_{\alpha}\left(v_{\alpha}\right) \mathrm{d} v_{\alpha}$,

and from this assign the probability of interacting with mass group $\alpha$ to be

$P_{\alpha}=\Gamma(r, v, \alpha) \Delta t$.

An encounter is deemed to have occurred, based on a random generated number $Z$ from a uniform distribution between 0 and 1 , if $Z$ is less than the total probability $P=\sum_{\alpha} P_{\alpha}$. The total probability is implicitly constrained to be less than unity by controlling the timestep size $\Delta t$, which is discussed in more detail in the subsequent section. In the case that $Z<P$, we select the third star $m_{3}$ based on the relative probabilities $P_{\alpha}$ and initiate our three-body integration scheme explained in Section 2.3.3.

\subsubsection{Time stepping}

We use a fourth-order Runge-Kutta integrator to evolve the effective acceleration introduced in Section 2.3 as well as the threebody interactions described in Section 2.3.3. During integrations, we utilize a time-step reduction scheme requiring that the accuracy of the solution does not vary by more than a tolerance of $\epsilon_{\mathrm{rk}}=10^{-5}$ when the time-step is halved. The initial integration time-step $\Delta t=\lambda(1+r) /(1+v)$ is dynamically determined to account for the position and velocity of the binary in the cluster, with $\lambda=0.1$ chosen to produce a time-step that is a fraction of the core dynamical time $r_{\mathrm{c}} / \bar{\sigma}$ for a binary at rest in the core. This time stepper accounts for the higher density in the core and the enlarged cross-section at small velocities. Although this choice of time-step is usually sufficient, some extra care needs to be taken when using $\Delta t$ in equation (23) to determine the encounter probability, so that the total probability does not exceed unity. To ensure that we correctly sample the encounter probabilities, by satisfying the constraint $P \ll 1$, we set $P_{\max }=0.1$ and enforce $P<P_{\max }$ by reducing the 
time-step $\Delta t$ when necessary. For the case $P>P_{\max }$, we decrease the succeeding time-step by letting $\lambda \rightarrow 0.9\left(P_{\max } / P\right) \lambda$. During the subsequent step, if $\lambda<\lambda_{0}$, where $\lambda_{0}=0.1$ is the fiducial value, and $P<P_{\max }$, we allow the time-step to increase slowly by setting $\lambda \rightarrow$ $1.1 \lambda$. Once $\lambda>\lambda_{\mathrm{o}}$ and the probability is satisfactorily small, which often occurs once the binary migrates out of the problematic dense region, we reset the time-step factor to $\lambda=\lambda_{0}$.

\subsubsection{Three-body interactions}

In the case of an encounter, the relative probabilities described in Section 2.3.1 determine the mass and velocity of the third object. We take this sampled velocity $v_{3}$ to be the velocity of the third body at infinity and calculate the relative velocity at infinity for the encounter from $v_{\infty}=\left|\boldsymbol{v}-\boldsymbol{v}_{3}\right|=\sqrt{v^{2}+v_{3}^{2}-2 v v_{3} \cos \chi}$. Given $v$ and the sampled $m_{3}$ and $v_{3}$, the relative velocity at infinity is determined up to the $\cos \chi$ term, which for an isotropic King model distribution function can be sampled from an analytic expression for $\chi \in[0, \pi]$ as in Sigurdsson \& Phinney (1995). With the mass of the third body and the relative velocity known, the maximum impact parameter is obtained from the cross-section for the encounter

$\pi p_{\max }^{2}=\sigma\left(\boldsymbol{v}, \boldsymbol{v}_{3}\right)=\pi p^{2}\left(1+\frac{2 G\left(m_{\mathrm{b}}+m_{3}\right)}{p v_{\infty}^{2}}\right)$,

with $p$ defined in equation (20). The actual impact parameter for the encounter is sampled from a uniform distribution in the area spanned by the maximum impact parameter $\pi p_{\max }^{2}$. The angles that comprise the remaining free variables necessary to specify the initial conditions are the projected true anomaly $f$ of the binary at the time that the incoming third body reaches pericentre, two angles $\theta$ and $\phi$ specifying the initial location of the third body with respect to the binary centre of mass, and the impact orientation $\psi$, which specifies the angle of the impact parameter in a plane transverse to the incoming velocity of the third body. These four angles are sampled in a manner consistent with Hut \& Bahcall (1983). With the initial conditions specified, the explicit integration is performed with a modified scheme based on Sigurdsson \& Phinney (1993).

We modify the original method of a fixed initial distance of the third star, at $R_{\text {in }}=20 a$, to one of variable distance to improve efficiency and to prevent the case of long three-body interactions that can exceed the cluster time-step. The addition of massive BHs introduces the possibility for wide binaries with orbital separations much greater than those for which the previous method was suited to handle. With a fixed choice for the distance of the third star from the binary, interactions such as distant flybys, which are the quickest to resolve computationally and have little impact on the binary, often take a time that exceeds the cluster evolution time-step and leads to the possibility of missing other probable encounters.

To represent the three-body system as an isolated one, and to reduce excessive time spent integrating long approaches, we require that $R_{\text {in }} \leq R_{\max }(n)$, where $R_{\max }(n)=(4 \pi n / 3)^{-1 / 3}$ is the 'interparticle' distance and is a function of the local density $n(r)$. Once $R_{\text {in }}$ is specified, we determine the relative velocity $v_{\text {in }}$ at $R_{\text {in }}$ based on the relative velocity at infinity. With these two quantities specified, we approximate the time for a flyby as $\delta t=2 R_{\text {in }} / v_{\text {in }}$. For the case in which $\delta t>\Delta t$, we let $R_{\text {in }} \rightarrow(\Delta t / \delta t) R_{\text {in }}$, calculate $v_{\text {in }}$ at the new initial distance and recompute the new estimated time. We repeat this procedure until the estimated time is roughly the same as the cluster time-step, $0.9<\delta t / \Delta t<1.1$. One important caveat is that this could lead to placing the third object too close to the binary, spoiling the assumption of an object at infinity approaching a well-defined binary. To address this issue, we maintain one extra condition on the initial distance specification, a consideration for which we are willing to forgo our time-step restrictions; that is, $\left(a / R_{\text {in }}\right)^{3} \leq 0.01$.

To increase the speed of the three-body integration, we move from a constant integration time-step to one that is dynamical. We choose a maximum time-step $\delta T_{\max }$ to be an arbitrarily small fraction $\epsilon=6.25 \times 10^{-3}$ of the binary period $T_{\mathrm{b}}$, i.e. $\delta T_{\max }=\epsilon T_{\mathrm{b}}$. At the end of each integration step, we update the time-step to $\delta T=\epsilon\left(r_{\min } / v_{\max }\right)$, where $r_{\min }$ is the minimum separation between any pair of the three objects and $v_{\max }$ is the largest velocity of the three bodies. This sets the time-step to the maximum allowable value in consideration of the need to resolve the dynamics of the three objects or any potentially bound pair. In some instances, a resonance can form a temporarily bound triple system, causing the integrator to reach the maximum number of steps $N_{\max }=2 \times 10^{6}$ or to exceed the arbitrarily specified maximum allowable time of $5 \Delta t$. Under these rare circumstances, we reinitialize the system with newly sampled initial angles and restart the integration. In addition to the occasional long-lasting semi-stable triples that form, there are also instances when a binary makes its way to the core where the average time-scale necessary to resolve the three body encounters begins to approach the time-scale for the evolution of the binary in the cluster. Since we calculate three-body encounters decoupled from the binary's evolution in the cluster, we are forced to terminate the run in such cases. As the cluster time-scale is inversely proportional to the cluster density, this situation is most likely to occur in the densest clusters. As a result of this time-scale termination criterion, although a similar number of realizations are performed for each cluster, the highest-density clusters have noticeably fewer runs than the lower-density clusters, as is observable in the rightmost column of Table 2. For standard encounters, which are often much shorter than the cluster time-step, we periodically check whether the interaction has resolved - according to the criteria discussed in the following section - and in the case that a new binary has formed, even temporarily, we update $\delta T_{\max }$ with the period of this new binary.

\subsubsection{Encounter resolution}

We first identify a potential binary amongst the triple system composed of the original binary, $m_{1}$ and $m_{2}$, and the third mass $m_{3}$, by selecting the pair with the largest gravitational binding energy. We refer to the masses in the potential binary as $\bar{m}_{1}$ and $\bar{m}_{2}$, which may no longer correspond to the original binary composed of $m_{1}$ and $m_{2}$. The remaining object, which is not part of the potential binary, is labelled $\bar{m}_{3}$ that is distinct from $m_{3}$. All unbarred variables represent the initial configuration where the third object is incoming, while barred variables refer to the system where a binary has been identified and the encounter is nearly resolved. The encounter can be resolved in three ways: (I) there is a well defined bound binary system with the third object unbound and moving off to infinity, (II) a merger has occurred, or (III) the system is completely ionized.

For case (I), we terminate the integration once the following criteria are all satisfied: (i) the third body has achieved the minimum required separation from the binary, $\mid \bar{r}_{3}-\left(\bar{m}_{1} \bar{r}_{1}+\bar{m}_{2} \bar{r}_{2}\right) /\left(\bar{m}_{1}+\right.$ $\left.\bar{m}_{2}\right) \mid>\max \left\{R_{\max }(n), 1.1 R_{\text {in }}\right\}$, (ii) the eccentricity $\bar{e}$ of $\bar{m}_{1}$ and $\bar{m}_{2}$ is less than unity, (iii) $\bar{m}_{1}$ and $\bar{m}_{2}$ are bound, specifically $\bar{E}_{\mathrm{b}}<0$, and (iv) $\bar{m}_{3}$ is unbound, i.e. $\bar{E}_{3}>0$. Here, $\bar{E}_{\mathrm{b}}$ is the total energy of the final binary and $\bar{E}_{3}$ is the total energy of the third body. In addition to the above requirements, to determine the final state of the 'isolated' binary, we continue the integration until the total potential 
Table 2. Summary of simulations. Listed are the $15 \mathrm{GCs}$ modelled for evolution along with the total cluster mass $M_{\mathrm{c}}$, squared velocity dispersion $\sigma^{2}$, the luminous core density $\rho_{\mathrm{L}}$, concentration $c$, and metallicity $Z$. The clusters are ordered by total mass. There are 39 independent models after taking into account the number of BHs retained by the cluster. Medium-to-high mass clusters can accommodate large $\mathrm{BH}$ populations without disrupting the listed structural parameters. The size of the BH population in lower-mass clusters is either (1) limited in number by the IMF or (2) by the ability of the cluster to maintain the model structural parameters in their presence; in these cases, the cluster is not used for evolutions and is omitted from the table. In the final column, we list the total number of evolutions performed for each case.

\begin{tabular}{|c|c|c|c|c|c|c|c|}
\hline Name & $M\left(\mathrm{M}_{\odot}\right)$ & $\sigma^{2}\left(\mathrm{~cm}^{2} \mathrm{~s}^{-2}\right)$ & $\rho_{\mathrm{L}}\left(\mathrm{L} \odot \mathrm{pc}^{-3}\right)$ & $c$ & $Z$ & $N_{\mathrm{BH}}$ & $N_{\text {runs }}$ \\
\hline Pal 13 & $5.12 \times 10^{3}$ & $8.10 \times 10^{9}$ & 1.45 & 0.66 & $2.6 \times 10^{-4}$ & 20 & 15232 \\
\hline NGC 6838 & $3.67 \times 10^{4}$ & $5.29 \times 10^{10}$ & $6.76 \times 10^{2}$ & 1.15 & $3.3 \times 10^{-3}$ & $\begin{array}{r}20 \\
200\end{array}$ & $\begin{array}{l}18364 \\
20430\end{array}$ \\
\hline NGC 6535 & $5.93 \times 10^{4}$ & $5.76 \times 10^{10}$ & $5.19 \times 10^{2}$ & 1.33 & $3.2 \times 10^{-4}$ & $\begin{array}{r}20 \\
200\end{array}$ & $\begin{array}{l}35865 \\
33561\end{array}$ \\
\hline NGC 6362 & $1.17 \times 10^{5}$ & $7.84 \times 10^{10}$ & $1.95 \times 10^{2}$ & 1.09 & $2.0 \times 10^{-3}$ & $\begin{array}{r}20 \\
200\end{array}$ & $\begin{array}{l}32544 \\
33798\end{array}$ \\
\hline NGC 5053 & $1.66 \times 10^{5}$ & $1.96 \times 10^{10}$ & 3.47 & 0.74 & $3.8 \times 10^{-5}$ & $\begin{array}{r}20 \\
200\end{array}$ & $\begin{array}{l}69058 \\
74681\end{array}$ \\
\hline NGC 6121 & $2.25 \times 10^{5}$ & $1.60 \times 10^{11}$ & $4.37 \times 10^{3}$ & 1.65 & $1.4 \times 10^{-3}$ & $\begin{array}{r}20 \\
200 \\
1000\end{array}$ & $\begin{array}{l}14429 \\
17884 \\
24667\end{array}$ \\
\hline NGC 5694 & $2.92 \times 10^{5}$ & $3.36 \times 10^{11}$ & $8.91 \times 10^{3}$ & 1.89 & $2.1 \times 10^{-4}$ & $\begin{array}{r}20 \\
200 \\
1000\end{array}$ & $\begin{array}{l}14029 \\
13382 \\
17445\end{array}$ \\
\hline NGC 6093 & $3.67 \times 10^{5}$ & $1.54 \times 10^{12}$ & $6.17 \times 10^{4}$ & 1.68 & $3.6 \times 10^{-4}$ & $\begin{array}{r}20 \\
200 \\
1000\end{array}$ & $\begin{array}{l}7435 \\
7019 \\
4645\end{array}$ \\
\hline NGC 5286 & $4.80 \times 10^{5}$ & $6.56 \times 10^{11}$ & $1.26 \times 10^{4}$ & 1.41 & $4.1 \times 10^{-4}$ & $\begin{array}{r}20 \\
200 \\
1000\end{array}$ & $\begin{array}{r}6761 \\
10032 \\
8196\end{array}$ \\
\hline NGC 6656 & $5.36 \times 10^{5}$ & $6.08 \times 10^{11}$ & $4.27 \times 10^{3}$ & 1.38 & $4.0 \times 10^{-4}$ & $\begin{array}{r}20 \\
200 \\
1000\end{array}$ & $\begin{array}{l}12539 \\
20993 \\
14832\end{array}$ \\
\hline NGC 1851 & $5.61 \times 10^{5}$ & $1.08 \times 10^{12}$ & $1.23 \times 10^{5}$ & 1.86 & $1.3 \times 10^{-3}$ & $\begin{array}{r}20 \\
200 \\
1000\end{array}$ & $\begin{array}{l}7189 \\
6950 \\
4563\end{array}$ \\
\hline NGC 6205 & $6.27 \times 10^{5}$ & $5.04 \times 10^{11}$ & $3.55 \times 10^{3}$ & 1.53 & $5.9 \times 10^{-4}$ & $\begin{array}{r}20 \\
200 \\
1000\end{array}$ & $\begin{array}{l}13444 \\
24899 \\
23583\end{array}$ \\
\hline NGC 6441 & $1.30 \times 10^{6}$ & $3.24 \times 10^{12}$ & $1.82 \times 10^{5}$ & 1.74 & $7.0 \times 10^{-3}$ & $\begin{array}{r}20 \\
200 \\
1000\end{array}$ & $\begin{array}{l}2388 \\
2439 \\
2463\end{array}$ \\
\hline NGC 104 & $1.45 \times 10^{6}$ & $1.21 \times 10^{12}$ & $7.59 \times 10^{4}$ & 2.07 & $3.8 \times 10^{-3}$ & $\begin{array}{r}20 \\
200 \\
1000\end{array}$ & $\begin{array}{r}9545 \\
10467 \\
8559\end{array}$ \\
\hline NGC 5139 & $2.64 \times 10^{6}$ & $2.82 \times 10^{12}$ & $1.41 \times 10^{3}$ & 1.31 & $5.9 \times 10^{-4}$ & $\begin{array}{r}20 \\
200 \\
1000\end{array}$ & $\begin{array}{l}13197 \\
17466 \\
23513\end{array}$ \\
\hline
\end{tabular}

energy between $\bar{m}_{3}$ and each mass in the binary is a fraction of the total energy of the system $E$, specifically

$\frac{G \bar{m}_{1} \bar{m}_{3}}{\left|\bar{r}_{1}-\bar{r}_{3}\right|}+\frac{G \bar{m}_{2} \bar{m}_{3}}{\left|\bar{r}_{2}-\bar{r}_{3}\right|}>0.05 E$.

In case (II), two of the bodies merge and the third body is either unbound or forms a new binary with the merger product. The criteria for mergers is based on the distance of nearest approach $d$ between two bodies during the three body encounter. In the case of a potential merger between two BHs, the merger criterion is $d \leq$ $R_{1}+R_{2}$. For the remaining merger situations, the criterion remains $d=f_{\text {tid }}\left(R_{1}+R_{2}\right)$, as adopted from Sigurdsson \& Phinney (1995), using the same value for $f_{\text {tid }}$ as introduced in Section 2.2.5. Our choice for $f_{\text {tid }}$ was selected, as it approximately separates the boundary of where hydrodynamical effects become important (e.g. Lee \&
Ostriker 1986; Benz \& Hills 1992). When this criterion is met, we assume a tidal encounter takes place. The merger is assumed to be a momentum conserving, impulsive, completely inelastic collision with no mass-loss (Davies, Benz \& Hills 1994). When a merger occurs between the $\mathrm{BH}$ companion and the third body, if the merger product remains bound to the $\mathrm{BH}$, this dynamically formed binary becomes our new 'test binary', which we continue to follow and evolve within the cluster. Similarly, if the BH merges with a third body and we still have a bound binary system, we again continue to follow this binary. However, if the $\mathrm{BH}$ becomes unbound by merging with another body or becomes unbound from a merger product, we handle the newly single $\mathrm{BH}$ as described in the subsequent section. In each of these cases, the position of the new binary, or single $\mathrm{BH}$, is updated by continuing along the original binary trajectory and the velocity is updated by converting from the 
three-body centre-of-mass frame, where the three-body integration is performed, back to the cluster frame.

The result of the encounter can also end in complete ionization, case (III). Ionization occurs in the case of ill-defined binaries that will inevitably be unbound if, given that all previous criteria are satisfied, either (a) the eccentricity of $\bar{m}_{1}$ and $\bar{m}_{2}$ satisfies $1-\bar{e}<$ $1 \times 10^{-7}$ or (b) $\left|\bar{r}_{1}-\bar{r}_{2}\right|>R_{\max }(n)$ is satisfied. Additionally, ionization occurs if $\bar{m}_{i} \bar{v}_{i}^{2}>2\left(\bar{m}_{i} \bar{m}_{j} /\left|\bar{r}_{i}-\bar{r}_{j}\right|+\bar{m}_{i} \bar{m}_{k} /\left|\bar{r}_{i}-\bar{r}_{k}\right|\right)$ is true for all masses at any time, with $i \neq j \neq k$ taking on values $\{1,2,3\}$. This last criterion is a straightforward definition for a totally unbound triple. In addition to these choices for ionization during three-body encounters, there is one other instance in which the binary can be dissociated. For very wide binaries, the encounters are dominated by repeated grazing encounters with low-mass stars, which tend to further widen the orbital separation. As a result, strong interactions become less likely and the binary will inevitably be dissociated by the increasing occurrence of these slowly ionizing encounters. For this reason, we use the encounter rate to define a maximum semimajor axis of dynamically formed binaries as

$a_{\max }(\Gamma)=\left(\frac{G m_{\mathrm{b}}}{3(2 \pi \Gamma)^{2}}\right)^{1 / 3}$,

which is equivalent to requiring a minimum of three orbits between encounters. Here, the total encounter rate $\Gamma=\sum_{\alpha} \Gamma(r, v, a)$ is a sum over the rate associated with each mass group defined by equation (22). The final criterion for ionization is then $a>\min \left\{a_{\max }(\Gamma), R_{\max }(n)\right\}$.

\subsubsection{Single $\mathrm{BHs}$}

As described in the previous section, a BH can become single due to three-body dynamics such as exchange, merger, or through the dismantling of a binary that exceeds our large $a$ or large $e$ criteria. In the case of a single $\mathrm{BH}$, we allow for the solitary $\mathrm{BH}$ to form a new binary by interacting with existing binaries within the cluster.

In order to accomplish this, we need to know the probability for the following encounter,

$\left(m_{1}, m_{2}\right)+m_{\mathrm{BH}} \rightarrow\left(m_{\mathrm{BH}}, m_{2}\right)+m_{1}$,

in which the $\mathrm{BH}$ exchanges with $m_{1}$ into a binary originally composed of masses $m_{1}$ and $m_{2}$. We also consider the possibility that $m_{\mathrm{BH}}$ and $m_{2}$ undergo an exchange, which contributes to the total probability that the $\mathrm{BH}$ will exchange into the binary. However, for conciseness in deriving the probability of exchange, we will focus specifically on the encounter described by equation (27), later adding the contribution from the reaction where the subscripts are interchanged. Unfortunately, we can no longer compute the probability for encounter as in Section 2.3.1, since we do not possess a distribution function for binaries. However, by considering the reverse reaction of equation (27), given by

$\left(m_{\mathrm{BH}}, m_{2}\right)+m_{1} \rightarrow\left(m_{1}, m_{2}\right)+m_{\mathrm{BH}}$,

and relating this to the one of interest, we can obtain the encounter probability for the $\mathrm{BH}$ to exchange into an existing binary in the same way that we compute encounters for a binary composed of a $\mathrm{BH}$ and a companion.

We use the seminumerical fit of Heggie, Hut \& McMillan (1996),

$\bar{\sigma}_{1,2}=\left(\frac{M_{23}}{M_{123}}\right)^{1 / 6}\left(\frac{m_{3}}{M_{13}}\right)^{7 / 2}\left(\frac{M_{123}}{M_{12}}\right)^{1 / 3}\left(\frac{M_{13}}{M_{123}}\right) g(2,3,1)$, as the dimensionless cross-section for a generically labelled single mass $m_{3}$ to exchange into a binary of masses $m_{1}$ and $m_{2}$ to form a new binary composed of $m_{3}$ and $m_{2}$, with $m_{1}$ being ejected. In this notation, uppercase masses represent the sum of the mass subscripts, i.e. $M_{i j}=m_{i}+m_{j}$. The coefficient $g(2,3,1)$ is a numerical fitting factor designed to improve the analytically derived fit. This dimensionless cross-section $\bar{\sigma}_{1,2}$ is related to the dimensionful cross-section for exchange $\Sigma_{1,2}$ through

$\bar{\sigma}_{1,2}=\frac{2\left|\boldsymbol{v}_{1,2}-\boldsymbol{v}_{3}\right|^{2}}{\pi G M_{123} a_{1,2}} \Sigma_{1,2}$.

The existing binaries that the $\mathrm{BH}$ is likely to encounter, which have remained intact in the cluster over long time-scales, can be considered 'hard'. These 'hard' binaries are characterized by having a binding energy $U_{\text {bin }}$ that exceeds the average energy of the other stars in the cluster $\left|U_{\text {bin }}\right|>\frac{1}{2} \bar{m} \bar{\sigma}^{2}$ and this is what allows them to stay intact over such long time-scales. In this case, we approximate the total encounter cross-section by the dominant gravitational focusing term in equation (21), explicitly:

$\sigma_{1,2} \simeq \frac{2 \pi G M_{123} a_{1,2}}{\left|\boldsymbol{v}_{1,2}-\boldsymbol{v}_{3}\right|^{2}}$

Finally, relating equations (30) and (31) allows us to express the cross-section for exchange in terms of the total encounter crosssection $\sigma_{1,2}$ through

$\Sigma_{1,2}=\left(\bar{\sigma}_{1,2} / 4\right) \sigma_{1,2}$.

Evidently, the dimensionless cross-section for exchange is related to the fractional probability that the total encounter ends in the specific exchange we previously described. Considering equation (31) and assuming the relative velocities are similar for the forward and reverse reactions, we can relate the forward and backward total cross-sections through $\sigma_{1,2}=\left(\frac{a_{1,2}}{a_{2,3}}\right) \sigma_{2,3}$. Since the energy given to the binary is comparable to the energy required to destroy it, $m_{1} m_{2} / a_{1,2} \sim m_{2} m_{3} / a_{2,3}$, we can recast the relation in terms of the masses alone:

$\sigma_{1,2}=\left(\frac{m_{1}}{m_{3}}\right) \sigma_{2,3}$

The cross-section for the specific exchange of $m_{3}$ for $m_{1}$ in terms of the total encounter cross-section of the original binary is found by substituting equation (33) into equation (32), yielding

$\Sigma_{1,2}=\left(\frac{\bar{\sigma}_{1,2} m_{1}}{4 m_{3}}\right) \sigma_{2,3}$.

By writing the exchange probability in terms of the post-exchange binary, we can now utilize the same procedure described in Section 2.3.1. In this formalism, $m_{3}$ represents the $\mathrm{BH}$ and we return to referring to this body as $m_{\mathrm{BH}}$, while $m_{1}$ goes to $m_{\alpha}$, a variable companion used for computing the relative probabilities for each mass group $\alpha$. First, we select a companion object $m_{2}$ for the $\mathrm{BH}$ on the left-hand side of equation (28). We obtain $m_{2}$ by sampling from the local number density and determine $a$ and $e$ for the binary as in Section 2.2.5. The probability of the encounter described by equation (27), where the $\mathrm{BH}$ exchanges places with $m_{\alpha}$ in a binary composed of $m_{2}$ and $m_{\alpha}$ is then,

$P_{\alpha, 2}=\Delta t \int\left(\frac{\bar{\sigma}_{\alpha, 2} m_{\alpha}}{4 m_{\mathrm{BH}}}\right) \sigma_{2, \mathrm{BH}}\left(\boldsymbol{v}, \boldsymbol{v}_{\alpha}\right)\left|\boldsymbol{v}-\boldsymbol{v}_{\alpha}\right| f_{\alpha}\left(v_{\alpha}\right) \mathrm{d} v_{\alpha}$. 
The usefulness of the manipulations in this section is most clearly seen by writing this in terms of equation (23):

$P_{\alpha, 2}=\left(\frac{\bar{\sigma}_{\alpha, 2} m_{\alpha}}{4 m_{\mathrm{BH}}}\right) P_{\alpha}$,

which in practice makes computing the exchange probabilities as easy as rescaling our standard encounter computations by the parenthetical factor. Since we also allow for the $\mathrm{BH}$ to exchange with $m_{2}$, we also consider the probability $P_{2, \alpha}=\left(\frac{\bar{\sigma}_{2, \alpha} m_{2}}{4 m_{\mathrm{BH}}}\right) P_{2}$.

We apply one final rescaling to account for the density of binaries that are of type $m_{2}$ and $m_{\alpha}$. We assume that the fraction of objects that are binaries $f_{\mathrm{b}}$ is constant throughout the cluster with the value specified by equation (16). The density of binaries is then $n_{\mathrm{b}}(r)=$ $\left(\frac{f_{\mathrm{b}}}{1+f_{\mathrm{b}}}\right) n(r)$, which is derived from equation (15). Additionally, we also assume that the fraction of binaries of a given type is constant at all cluster radii, $n_{i j}(r)=f_{i / j} n_{\mathrm{b}}(r)$. Here, $f_{i / j}$ represents the fraction of binaries that have a star of type $i$ and a star of type $j$, e.g. $f_{\mathrm{NS} / \mathrm{MS}}$ is the fraction of all binaries that are composed of an NS and an MS star. For binaries composed of only MS or WD, we use values of $f_{\mathrm{MS} / \mathrm{MS}}=0.23, f_{\mathrm{MS} / \mathrm{WD}}=0.44$, and $f_{\mathrm{WD} / \mathrm{WD}}=0.32$ (Fregeau et al. 2009). The remaining one per cent of binaries contain at least one $\mathrm{BH}$ or NS, for which we compute the binary fraction through $f_{i / j}=0.01\left(\frac{N_{i}}{N}\right)\left(\frac{N_{\mathrm{j}}}{N_{\mathrm{BH}+\mathrm{NS}}}\right)$, where $i$ can be any object type, $j$ is limited to $\mathrm{BH}$ or NS, $N$ is the total number of objects in the cluster, and $N_{\text {BH }+ \text { NS }}$ is the total number of BHs and NSs.

The final total probability for the $\mathrm{BH}$ to exchange into a binary, given the sampled mass $m_{2}$, is then

$P_{\text {exch }}(r)=\sum_{\alpha} n_{\alpha 2}(r)\left(\frac{P_{\alpha, 2}}{n_{\alpha}(r)}+\frac{P_{2, \alpha}}{n_{2}(r)}\right)$.

Here, we divide out the respective local density picked up in the integration of the distribution function in order to enforce our assumption of a uniform binary fraction. If an exchange is determined to occur based on this total probability, we select a specific binary for the encounter based on the relative probabilities of exchange for each mass group $m_{\alpha}$. With a binary in hand, we initiate our threebody system, which is run until we get the proper outcome dictated by the encounter cross-section - i.e. that $m_{\mathrm{BH}}$ exchanges with the appropriate mass in the binary.

\section{SIMULATIONS}

We present 698486 realizations from $15 \mathrm{GC}$ models with total masses in the range of $5.12 \times 10^{3}-2.64 \times 10^{6} \mathrm{M}_{\odot}$, velocity dispersions covering $9 \times 10^{4}-1.8 \times 10^{6} \mathrm{~cm} \mathrm{~s}^{-1}$, core densities of $1.45-1.23 \times 10^{5} \mathrm{pc}^{-3}$, and concentrations spanning $0.66-2.07$. The simulations are summarized in Table 2, which includes the catalogue name for the modelled cluster, total mass, velocity dispersion squared, central luminosity density, concentration, metallicity, the number of retained $\mathrm{BHs}$ in the model, and the total number of completed runs. The simulations are run for $t=10^{10} \mathrm{yr}$ or until the single/binary is ejected from the cluster, when $r>r_{\mathrm{t}}$.

\subsection{Structural parameters}

In our framework, a GC's structure is determined by four parameters: the total cluster mass $M_{\mathrm{c}}$, the core velocity dispersion $\sigma$, the core luminosity density $\rho_{\mathrm{L}}$, and the concentration $c$. McLaughlin (2000) finds that GCs described by single-mass isotropic King models are fully defined by four independent physical parameters: the

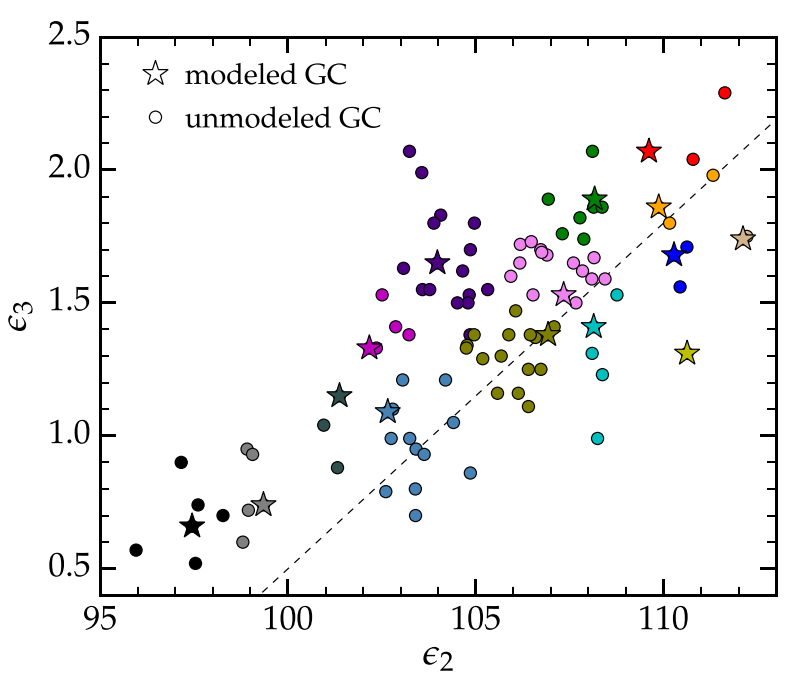

Figure 3. The distribution of non-core-collapsed Milky Way GCs in a face-on view of the Fundamental Plane. The colour of each unmodelled GC (marked by circles) indicates the corresponding modelled GC (marked by stars) that serves as its proxy for determining the properties of the ejected binaries. The plane is defined by $\epsilon_{2}=2.05 \log _{10} E_{\mathrm{b}}^{*}+\log _{10} L$ and $\epsilon_{3}=c$, with the dashed line corresponding to the fit $\epsilon_{3}=-12.5+0.13 \epsilon_{2}$. Here, $c$ is the concentration, $L$ is the total luminosity, and $E_{\mathrm{b}}^{*}$ is an additional parameter related to $L$ (see Section 3.2 for additional details).

mass-to-light ratio $\Upsilon_{\mathrm{v}, 0}$, total binding energy $E_{\mathrm{b}}$, central concentration $c$, and total luminosity L. Furthermore, McLaughlin (2000) shows that Milky Way GCs lie in a 'Fundamental Plane' and thus can be fully described by just two independent parameters, $c$ and $L$. A face-on view of the Fundamental Plane is defined by the axes $\epsilon_{2}=2.05 \log _{10} E_{\mathrm{b}}^{*}+\log _{10} L$ and $\epsilon_{3}=c$. The apparent dependence on the third quantity $\log _{10} E_{\mathrm{b}}^{*}$ is due to a rotation in the larger threedimensional space in order to remove projection effects. However, this is reconciled by showing that this third parameter, $E_{\mathrm{b}}^{*}$, is fully described by the luminosity, such that $E_{\mathrm{b}}^{*}(L)$ (McLaughlin 2000). With the space of physical clusters reduced to the Fundamental Plane, we determine a representative group of 15 Milky Way clusters by sampling from the two-dimensional distribution. A face-on view of the Fundamental Plane is given in Fig. 3, which includes all GCs from the Harris catalogue (Harris 1996, 2010 edition) for which observed concentrations are available. We omit clusters identified in the catalogue as core collapsed, since these are not generally well described by King models. This includes those with $c=2.5$, an arbitrary value assigned to clusters in the catalogue with central density cusps indicative of core collapse. There are 125 Milky Way GCs remaining after core-collapse pruning; of these, 15 GCs are chosen as representative models, in an attempt to properly cover the fundamental parameter space. The 15 Milky Way GC models representative of the 125 Milky Was GCs are described in Table 2 and represented by stars in Fig. 3 to visualize our coverage of the fundamental parameter space.

As stated in Section 2.1, our input parameters for specifying the structure of a cluster are the core velocity dispersion $\bar{\sigma}$, the central density $n_{0}$, and the King parameter $W_{0}$. The mean core velocity dispersion $\bar{\sigma}$ is chosen to be the observed value listed in the Harris catalogue. The core number density $n_{\mathrm{o}}$ is adjusted until the central luminosity density $\rho_{\mathrm{L}}$ is consistent with observation. Finally, the King parameter $W_{\mathrm{o}}$, which sets the depth of the potential, is varied until the cluster has the desired total mass $M_{\mathrm{c}}$ and concentration $c$. 
Once we have a model for a given GC, we add BHs by increasing the fraction of retained $\mathrm{BHs} f_{\mathrm{r}_{\mathrm{BH}}}$, where a value of unity corresponds to retention of all $\mathrm{BHs}$ produced according to the IMF. For a given number of $\mathrm{BHs}$ in the cluster, we use the parameter $f_{s}$ in equation (18) to adjust the $\mathrm{BH}$ velocity dispersion such that the overall structure of the cluster is unaffected by the presence of a significant number of BHs. However, we find that there is a limit to the number of BHs each cluster can harbour. For the lowest-mass clusters, such as Pal 13, setting the retention factor to unity, $f_{\mathrm{r}_{\mathrm{BH}}}=1$, in order to maximize the number of BHs retained by the cluster produces a peak number of $\sim 20 \mathrm{BHs}$. In this case, the number of BHs retained by the cluster is inherently limited by its structure. More generally, for lower-mass clusters that allow for more BHs, the large number of BHs can become problematic as they become a more significant part of the total mass of the cluster. As the fraction of the total mass in BHs increases, the BHs begin to affect the structural parameters such that no set of initial parameters exists that satisfy the observed structure of the GC. We find that for many of the lower-mass clusters we are only able to simulate populations of 20 or $200 \mathrm{BHs}$ (cf. Table 2).

\subsection{Galactic evolution}

The GC evolution models, described in detail in Section 2, compute the properties of the $\mathrm{BH}$ binaries at the moment they are ejected from a GC. Determining the present-day properties of potentially observable, ejected $\mathrm{BH}$ binaries requires further modelling that tracks both the evolution of ejected binaries in the Milky Way potential and the internal evolution of each binary. In this section, we describe Monte Carlo models for the subsequent evolution of the ejected binaries that are seeded with results from our GC models.

\subsubsection{Globular cluster orbits}

We first build a sample of GCs to include in our Galactic evolution simulations. The orbit of a cluster is specified by its location on the sky (right ascension and declination), distance from the Sun $\mathrm{D}_{\odot}$, radial velocity $v_{r}$, and proper motion $\mu_{\alpha}$ and $\mu_{\delta}$. Of the 125 non-core-collapsed GCs in the Harris catalogue (Harris 1996, 2010 edition), we are able to find literature values for the orbital parameters of 106 of these clusters in the catalogues of Moreno, Pichardo \& Velázquez (2014) and Kharchenko et al. (2013). For clusters appearing in both catalogues, we use the values given in Moreno et al. (2014).

To begin each realization in our Monte Carlo ensemble, we initialize the GC orbits by sampling the uncertainty in their current positions and velocities. We assume normally distributed errors and use the quoted uncertainties in $v_{r}, \mu_{\alpha}$, and $\mu_{\delta}$. Following Krauss $\&$ Chaboyer (2003), we assume a 6 per cent error in $\mathrm{D}_{\odot}$. After the orbit is specified, we integrate it $10 \mathrm{Gyr}$ backwards in time, corresponding to the duration of our GC dynamical simulations.

The orbits of the GCs, and the ejected binaries, are integrated using the PYTHON Galactic dynamics library GALPY (Bovy 2015). We model the Milky Way gravitational potential using the builtin mWPOTENTIAL2014. The potential includes contributions from the Galactic bulge, disc, and halo, which have been fit to observational data to provide a realistic model of the Milky Way potential. The physical scale of the potential is set using the distance from the centre of the Galaxy to the Sun and the circular velocity of the Sun, which we set to $8 \mathrm{kpc}$ and $220 \mathrm{~km} \mathrm{~s}^{-1}$, respectively. For all calculations, we use the DOPR54_c integrator, a fast implementation of a high-order Dormand-Prince method included with GALPY.
Now that we have calculated the positions and velocities of the Milky Way GCs during the past $10 \mathrm{Gyr}$, the next step is to determine the properties of any potential BH-LMXBs ejected by these clusters. Since our dynamical simulations only include a subset of the Galactic GCs, we use the results from the 15 GCs simulated in Table 2 as proxies for the ejected binary populations produced by the remaining 110 clusters in our Galactic evolution models. For each of the unmodelled clusters, a proxy cluster is selected by finding the nearest simulated cluster in the Fundamental Plane (see Section 3.1). Specifically, we find $\min \left[\left(\epsilon_{2, \mathrm{i}}^{\prime}-\epsilon_{2, \mathrm{j}}^{\prime}\right)^{2}+\left(\epsilon_{3, \mathrm{i}}^{\prime}-\epsilon_{3, \mathrm{j}}^{\prime}\right)^{2}\right]$, where the $i$ index runs over all 106 clusters in the Galactic evolution models, the $j$ index runs over the 15 clusters included in our GC dynamics models, and the primes denote the normalized versions of $\epsilon_{2}$ and $\epsilon_{3}$ restricted to the range [0,1]. Fig. 3 shows the proxy cluster chosen for each GC, by assigning the same colour marker to each GC as the colour of the proxy cluster used, which are marked by coloured stars. To ensure the robustness of this method for choosing a proxy cluster, we assign a proxy by two additional methods. One secondary method is to assign the proxy cluster based on the minimum distance in the Fundamental Plane using the unnormalized axes $\epsilon_{2}$ and $\epsilon_{3}$. The second alternative is by identifying the most similar cluster using the structural parameters $M_{\mathrm{c}}, \sigma$, and $\rho_{\mathrm{L}}$ weighted according to the strengths of the correlations between these parameters and the ejected binary populations, which are explored in 4.1. Selecting the proxy cluster by any of these three methods gives similar results in our Galactic evolution models. In fact, all three methods will select the same proxy cluster for all but $\sim 15$ of the 110 unmodelled GCs in our study. In what follows, we discuss models that use the scaled distance in the Fundamental Plane to assign the proxy cluster.

\subsubsection{The ejected binaries}

The output of our GC dynamical simulations describes the properties of the BH binaries ejected from GCs. To model the present-day population of BH-LMXBs that are ejected from GCs, we use as inputs for our Galactic evolution models: the ejection time $t_{\mathrm{ej}}$, ejection velocity $v_{\mathrm{ej}}$, and the properties of the binary, the semimajor axis $a$, eccentricity $e$, the mass of the BH primary $m_{1}$, and the mass of the companion $m_{2}$. This is accomplished by constructing empirical cumulative distribution functions (CDFs) of these quantities for each of the 37 sets of parameters listed in Table 2, and then sampling these distributions in our Monte Carlo models. We assume that the ejection time, ejection velocity, and binary properties are independent and sample the marginal distributions of each.

In the GC dynamical models, $a, e, t_{\mathrm{ej}}$, and $v_{\mathrm{ej}}$ are treated as continuous variables. As such, we are able to sample the CDFs for these quantities directly. We fit cubic splines to the empirical CDFs and invert the distributions by interpolation. The GC dynamical models treat $m_{1}$ and $m_{2}$ as discrete quantities, which fall into the mass bins shown in Table 1. In our Galactic evolution models, however, we want to consider continuous masses. To accomplish this, we first determine an object's mass bin by sampling the discrete CDF output by the dynamical simulations. Next, we sample the mass distribution within that bin using the EMF described in Section 2.2.1. Using these CDFs, we are able to generate sample populations of the $\mathrm{BH}$ binaries ejected by the $106 \mathrm{GCs}$ in our Galactic evolution simulations.

During each realization, for each cluster, we first determine the number of binaries that the cluster will eject during the $10 \mathrm{Gyr}$ simulation by sampling a Poisson distribution with rate parameter 
Table 3. Expected number of binary ejections. For each cluster and number of retained $\mathrm{BHs}$, we list the exact number of $\mathrm{BHs}$ in the cluster along with the expected number of ejections over the cluster lifetime for three binary types: BH-NC, BH-NS, and BH-BH. The clusters follow the same order as Table 2, sorted according to increasing total cluster mass. The values of $N_{\mathrm{BH}}$ are non-integer values as a consequence of modelling the population with a smooth distribution function.

\begin{tabular}{|c|c|c|c|c|}
\hline Name & $N_{\mathrm{BH}}$ & $\mathrm{BH}-\mathrm{NC}$ & BH-NS & $\mathrm{BH}-\mathrm{BH}$ \\
\hline Pal 13 & 19.64 & 3.14 & $7.74 \times 10^{-3}$ & $1.40 \times 10^{-1}$ \\
\hline \multirow[t]{2}{*}{ NGC 6838} & 20.61 & $6.33 \times 10^{-1}$ & $3.59 \times 10^{-2}$ & $5.08 \times 10^{-1}$ \\
\hline & 174.55 & $2.56 \times 10^{1}$ & $2.39 \times 10^{-1}$ & 2.67 \\
\hline \multirow[t]{2}{*}{ NGC 6535} & 19.89 & $2.35 \times 10^{-1}$ & $1.72 \times 10^{-2}$ & $3.64 \times 10^{-1}$ \\
\hline & 198.95 & 5.12 & $1.24 \times 10^{-1}$ & 2.08 \\
\hline \multirow[t]{2}{*}{ NGC 6362} & 20.22 & $1.61 \times 10^{-1}$ & $6.83 \times 10^{-3}$ & $2.31 \times 10^{-1}$ \\
\hline & 199.33 & 1.07 & $2.36 \times 10^{-2}$ & 1.55 \\
\hline \multirow[t]{2}{*}{ NGC 5053} & 21.71 & $2.04 \times 10^{-2}$ & $3.14 \times 10^{-4}$ & $7.31 \times 10^{-2}$ \\
\hline & 199.65 & $1.79 \times 10^{-1}$ & $2.67 \times 10^{-3}$ & $4.96 \times 10^{-1}$ \\
\hline \multirow[t]{3}{*}{ NGC 6121} & 20.70 & $3.11 \times 10^{-1}$ & $6.31 \times 10^{-2}$ & $4.96 \times 10^{-1}$ \\
\hline & 200.53 & 1.74 & $3.03 \times 10^{-1}$ & 2.66 \\
\hline & 1039.16 & $1.02 \times 10^{2}$ & 1.43 & 8.17 \\
\hline \multirow[t]{3}{*}{ NGC 5694} & 20.49 & $2.29 \times 10^{-1}$ & $1.18 \times 10^{-1}$ & $7.49 \times 10^{-1}$ \\
\hline & 200.39 & 1.54 & 1.02 & 4.19 \\
\hline & 1001.94 & $3.21 \times 10^{1}$ & 2.87 & $1.54 \times 10^{1}$ \\
\hline \multirow[t]{3}{*}{ NGC 6093} & 19.85 & $1.01 \times 10^{-1}$ & $4.81 \times 10^{-2}$ & $3.42 \times 10^{-1}$ \\
\hline & 198.31 & 1.13 & $3.67 \times 10^{-1}$ & 2.66 \\
\hline & 1004.51 & $1.23 \times 10^{1}$ & 2.38 & $1.31 \times 10^{1}$ \\
\hline \multirow[t]{3}{*}{ NGC 5286} & 12.29 & $6.00 \times 10^{-2}$ & $2.36 \times 10^{-2}$ & $1.91 \times 10^{-1}$ \\
\hline & 198.28 & $9.29 \times 10^{-1}$ & $5.93 \times 10^{-2}$ & 2.08 \\
\hline & 787.45 & 4.42 & $3.84 \times 10^{-1}$ & 5.48 \\
\hline \multirow[t]{3}{*}{ NGC 6656} & 19.80 & $6.79 \times 10^{-2}$ & $1.42 \times 10^{-2}$ & $2.57 \times 10^{-1}$ \\
\hline & 205.86 & $4.22 \times 10^{-1}$ & $8.83 \times 10^{-2}$ & 1.74 \\
\hline & 1000.35 & 3.10 & $2.02 \times 10^{-1}$ & 5.09 \\
\hline \multirow[t]{3}{*}{ NGC 1851} & 20.76 & $8.37 \times 10^{-2}$ & $4.91 \times 10^{-2}$ & $4.74 \times 10^{-1}$ \\
\hline & 203.71 & $8.79 \times 10^{-1}$ & $4.98 \times 10^{-1}$ & 3.09 \\
\hline & 1039.94 & $1.98 \times 10^{1}$ & 1.82 & $1.03 \times 10^{1}$ \\
\hline \multirow[t]{3}{*}{ NGC 6205} & 20.10 & $6.13 \times 10^{-2}$ & $1.79 \times 10^{-2}$ & $2.62 \times 10^{-1}$ \\
\hline & 199.58 & $4.25 \times 10^{-1}$ & $5.61 \times 10^{-2}$ & 1.70 \\
\hline & 998.62 & 1.61 & $1.27 \times 10^{-1}$ & 5.36 \\
\hline \multirow[t]{3}{*}{ NGC 6441} & 20.98 & $3.51 \times 10^{-2}$ & $1.76 \times 10^{-2}$ & $3.16 \times 10^{-1}$ \\
\hline & 212.57 & $9.59 \times 10^{-1}$ & $8.72 \times 10^{-2}$ & 1.57 \\
\hline & 1010.37 & 3.69 & $8.20 \times 10^{-1}$ & 4.72 \\
\hline \multirow[t]{3}{*}{ NGC 104} & 22.49 & $6.60 \times 10^{-2}$ & $3.06 \times 10^{-2}$ & $4.49 \times 10^{-1}$ \\
\hline & 222.95 & 1.09 & $4.47 \times 10^{-1}$ & 2.89 \\
\hline & 979.55 & 3.09 & 2.52 & 8.41 \\
\hline \multirow[t]{3}{*}{ NGC 5139} & 20.84 & 0.00 & 0.00 & $2.53 \times 10^{-2}$ \\
\hline & 207.50 & $1.19 \times 10^{-2}$ & 0.00 & $1.19 \times 10^{-1}$ \\
\hline & 1009.04 & 0.00 & 0.00 & $2.57 \times 10^{-1}$ \\
\hline
\end{tabular}

$\left\langle N_{\mathrm{ej}}\right\rangle$ (the third column of Table 3 ). Once we have determined the number $N_{\text {bin }}$ of ejected binaries, we draw $N_{\text {bin }}$ samples from the $a$, $e, m_{1}, m_{2}, t_{\mathrm{ej}}$, and $v_{\mathrm{ej}}$ distributions.

Since the internal evolution of a binary is independent of its orbit in the Galaxy, we separately compute the full internal evolution of the binary using the rapid binary population synthesis code BSE described in Hurley, Tout \& Pols (2002) with the updates described in Clausen et al. (2012) and Lamberts et al. (2016). BSE combines interpolated stellar evolution models with recipes for mass transfer and other binary evolution processes to enable rapid modelling of a binary system's lifetime. Binary population synthesis calculations employ parametrized models to describe poorly understood processes in binary evolution. In our BSE runs, we assume that stable mass transfer is conservative. Additionally, we use a commonenvelope efficiency parameter of 1.0 and include the effects of tidal circularization.
We use each set of $a, e, m_{1}, m_{2}$ as the initial conditions for a BSE run. When handling the binary stellar evolution to determine which ejected binaries become mass transferring, we discard a small number of binaries that would have begun mass transfer within the cluster. The internal evolution of these tight binaries are coupled to their dynamical evolution within the cluster in a complex manner. Since these effects are not accounted for in our code, we do not include them in our results. For the remaining binaries, we set the companion star's metallicity to that of its parent $\mathrm{GC}$ and its age to $t_{\mathrm{ej}}$. The latter has little effect because most of the ejected stars have lifetimes that exceed $10 \mathrm{Gyr}$. The binary is evolved for $t_{\mathrm{evol}}=10 \mathrm{Gyr}-t_{\mathrm{ej}}$, i.e. to the present day. Systems are discarded if the companion star is not overflowing its Roche lobe and transferring mass to the $\mathrm{BH}$ at the end of the simulation. For each mass transferring binary, we determine the position $\boldsymbol{r}_{\mathrm{GC}}$ and velocity $\boldsymbol{v}_{\mathrm{GC}}$ of its parent GC at $t_{\mathrm{ej}}$. We initialize an orbit for the ejected binary at $\boldsymbol{r}_{\mathrm{GC}}$ and $\boldsymbol{v}_{\mathrm{GC}}+\boldsymbol{v}_{\mathrm{ej}}$, assuming that the binaries are ejected isotropically. With the initial conditions determined, we then evolve these binaries using GALPY to determine their positions at the present day.

Our Galactic evolution models consider three BH-retention scenarios. In the first, we assume that most BHs are ejected and use the results from our GC dynamics models with $N_{\mathrm{BH}}=20$. We refer to this set of models as MIN. In the second case, referred to as 200, we assume moderate $\mathrm{BH}$ retention, using the results from our GC dynamics models with $N_{\mathrm{BH}}=200$. Finally, in a case denoted MAX, we consider significant $\mathrm{BH}$ retention by utilizing the $\mathrm{GC}$ dynamics models with $N_{\mathrm{BH}}=1000$. In cases where we are unable to generate a background cluster model with the appropriate $N_{\mathrm{BH}}$, we use the results from the model with nearest $N_{\mathrm{BH}}$ simulated for that same cluster. We compute $10^{4}$ realizations for the MIN and 200 cases and $5 \times 10^{3}$ realizations for the MAX case.

\section{RESULTS}

Our simulations of binary-single star interactions in GCs provide us with statistical properties of the ejected $\mathrm{BH}$ binaries they produce including ejection time $t_{\mathrm{ej}}$, ejection velocity $v_{\mathrm{ej}}$, the orbital properties $a$ and $e$, and the component masses $m_{1}$ and $m_{2}$. Combining these results with the methods described in Section 3.2, we obtain predictions for the distribution and properties of the Galactic population of BH-LMXBs produced by GCs. Additionally, the simulations allow us to explore merger events involving BHs such as gravitational radiation driven mergers, both in the cluster and post-ejection, as well as those mergers that occur during three-body encounters. We describe these results in detail below.

\subsection{Ejected BH binaries}

We find that the number of ejected binaries and the properties of these binaries are strongly affected by the GC structure and the number of retained BHs. In Table 3, we list the expected number of ejected $\mathrm{BH}$ binaries over the life of each cluster, listed in order of increasing mass, including the exact number of BHs in each cluster. The ejected BH-binary expectation value is well described by the number of retained $\mathrm{BHs} N_{\mathrm{BH}}$ and the two characteristic variables that define the Fundamental Plane of GCs (see Fig. 3), namely the total cluster mass $M_{\mathrm{c}}$ and the concentration $c$. In Fig. 4, we plot the expected number of ejected $\mathrm{BH}$ binaries as a function of the three characteristic variables: $N_{\mathrm{BH}}, M_{\mathrm{c}}$, and $c$.

The most important structural variable that impacts the ejected binary properties is the cluster mass. The total cluster mass enforces a minimum energy needed to escape, which the binary must gain 


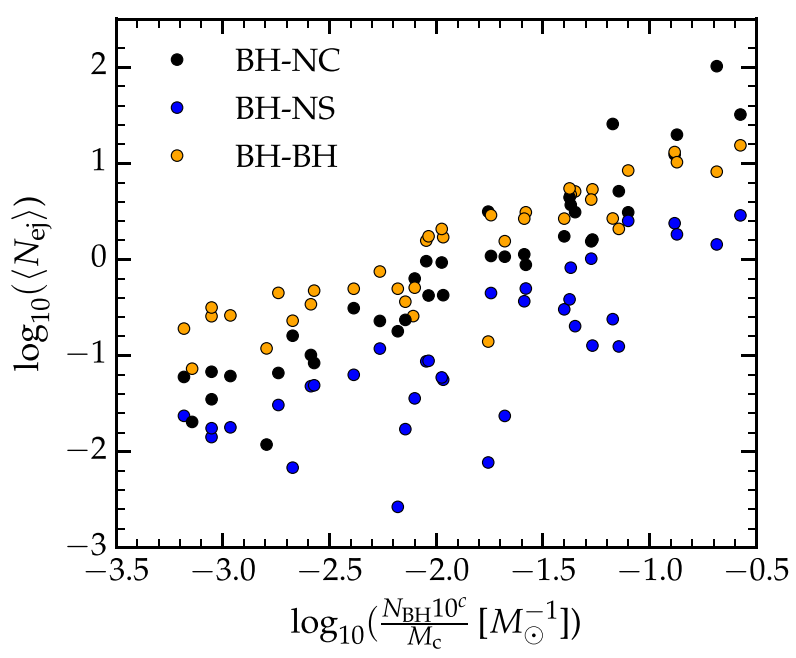

Figure 4. Expected number of binary ejections $\left\langle N_{\mathrm{ej}}\right\rangle$ as a function of the number of retained BHs $N_{\mathrm{BH}}$, concentration $c$, and total cluster mass $M_{\mathrm{c}}$. The number of binaries ejected over the life of the cluster is well described by the two characteristic variables of the Fundamental Plane, $c$ and $M_{\mathrm{c}}$, along with the number of $\mathrm{BHs}$ retained by the cluster.

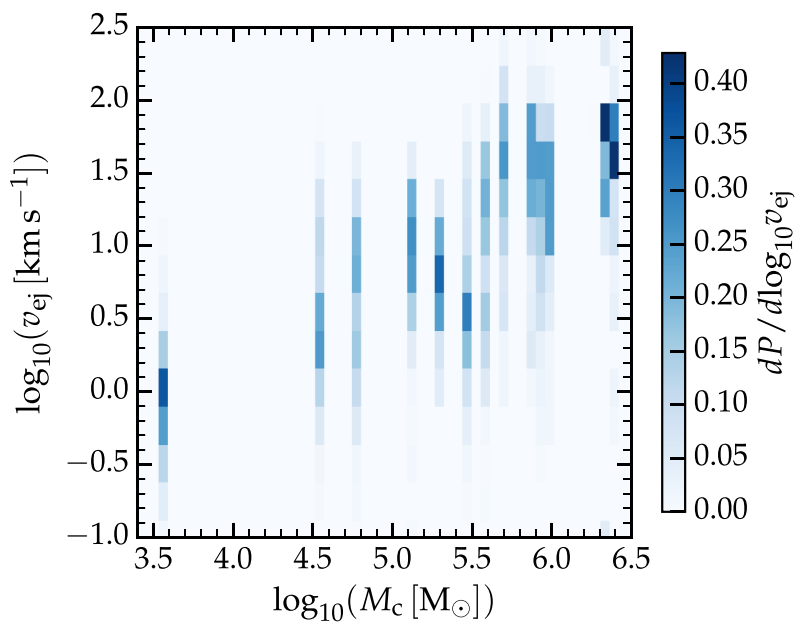

Figure 5. The distributions of ejection velocities $v_{\mathrm{ej}}$ as a function of the total cluster mass $M_{\mathrm{c}}$ for the ejected binaries. Each vertical bar represents the distribution of $v_{\mathrm{ej}}$ for the corresponding mass $M_{\mathrm{c}}$ and is normalized such that the integral over $\log _{10} v_{\text {ej }}$ in each mass bin yields unity. The binary velocity fluctuates due to random encounters with other stars in the cluster until the binary acquires a high enough recoil velocity to exceed the minimum ejection velocity, which is determined by the cluster mass. The increase in the necessary velocity for escape is apparent in the increasing mean value of each $v_{\mathrm{ej}}$ distribution.

through repeated encounters. In order for a binary to escape from the cluster, it must acquire a recoil velocity from a final three-body encounter high enough to climb out of the cluster gravitational potential. In Fig. 5, we show the distribution of the ejected binary velocities as a function of cluster mass, where the influence of the mass of the cluster on the ejection velocity is apparent.

The expected number of ejections is then higher for lower-mass clusters due to the lower escape velocities associated with these clusters, as is visible in Fig. 4. To decouple this statement from the additional variables in Fig. 4, it can also be observed in Table 3 (which is ordered by increasing mass) that for a fixed number of retained $\mathrm{BHs}$, the expected number of ejections scales with the cluster mass.

The mechanism through which the binary converts binding energy to kinetic energy is easiest to understand in the three-body centre of mass frame, where we perform our integration for encounters. After an encounter, the final relative velocity at infinity is given by

$\bar{v}_{\infty}^{2}=\frac{m_{3}\left(m_{1}+m_{2}\right)}{\bar{m}_{3}\left(\bar{m}_{1}+\bar{m}_{2}\right)} v_{\infty}^{2}+\frac{2 M_{123}}{\bar{m}_{3}\left(\bar{m}_{1}+\bar{m}_{2}\right)}\left(U_{\text {bin }}-\bar{U}_{\text {bin }}\right)$,

where $U_{\text {bin }}=-\frac{G m_{1} m_{2}}{a}$ is the binding energy of the binary and all unbarred quantities represent the initial binary before encountering $m_{3}$, while barred quantities represent the final binary and $\bar{m}_{3}$ is the ejected mass. In the case of no exchange, and utilizing $\Delta a \equiv \bar{a}-a$, equation (38) reduces to

$\bar{v}_{\infty}^{2}=v_{\infty}^{2}-\frac{2 M_{123}}{m_{3} m_{\mathrm{b}}}\left(\frac{G m_{1} m_{2} \Delta a}{a^{2}}\right)$.

In this frame, the binary velocity is related, through conservation of momentum, to the relative velocity simply by $v_{b}=\frac{m_{3}}{M_{123}} v_{\infty}$. The change in the kinetic energy, $\Delta T \equiv \bar{T}-T$, of the binary is then

$\Delta T=-\frac{G m_{1} m_{2} m_{3}}{M_{123}}\left(\frac{\Delta a}{a^{2}}\right)$.

The amount by which the semimajor axis changes in an average encounter, where the semimajor axis is reduced without exchange, is proportional to the semimajor axis, $\Delta a \approx-\epsilon a$, with $\epsilon$ in the range $\sim[0,0.6]$ (Sigurdsson \& Phinney 1993). Using this relation, and assuming a binary with constant $m_{1}$ and $m_{2}$, equation (40) reduces to

$\Delta T \propto \frac{m_{3}}{M_{123}} \frac{\epsilon}{a}$,

yielding a simple relation that describes the gain in kinetic energy in terms of the constant fractional change in the semimajor axis $\epsilon$ and the ratio of the third body to the total mass of the three-body system. Additionally, equation (41) shows that this change in kinetic energy becomes more efficient as the semimajor axis decreases, converting more energy from binding to kinetic after each encounter that shrinks the binary's orbit. After repeated interactions, the increase in velocity due to the decrease in $a$ becomes more substantial and the binary can eventually reach the necessary velocity to escape.

We can directly relate the necessary gain in kinetic energy to the change in binding energy $\Delta U=\bar{U}_{\text {bin }}-U_{\text {bin }}$, by simply rearranging equation (38) and assuming no exchange of masses, which yields

$\Delta T=-\frac{m_{3}}{M_{123}} \Delta U$.

In the process of the binary increasing its kinetic energy, the binding energy becomes more negative. Since the higher-mass clusters tend to hold on to the binaries longer, this strict minimum kinetic energy for ejection is manifest in the more negative-valued binding energy of the binaries it ejects. It follows from this, that on average, the semimajor axes of the binaries ejected from more massive clusters tend to be smaller. This is confirmed by Fig. 6, which depicts the distribution of orbital separations as a function of cluster mass.

In addition to the increase in the expected number of ejected binaries in lower-mass clusters, the total number of expected ejections also increases with an increase in the number of BHs. While the number of ejections is expected to increase with the number of $\mathrm{BHs}$, interestingly, the fraction of ejected binaries composed of a $\mathrm{BH}$ and non-compact (BH-NC) object also grows with the number of $\mathrm{BHs}$ 


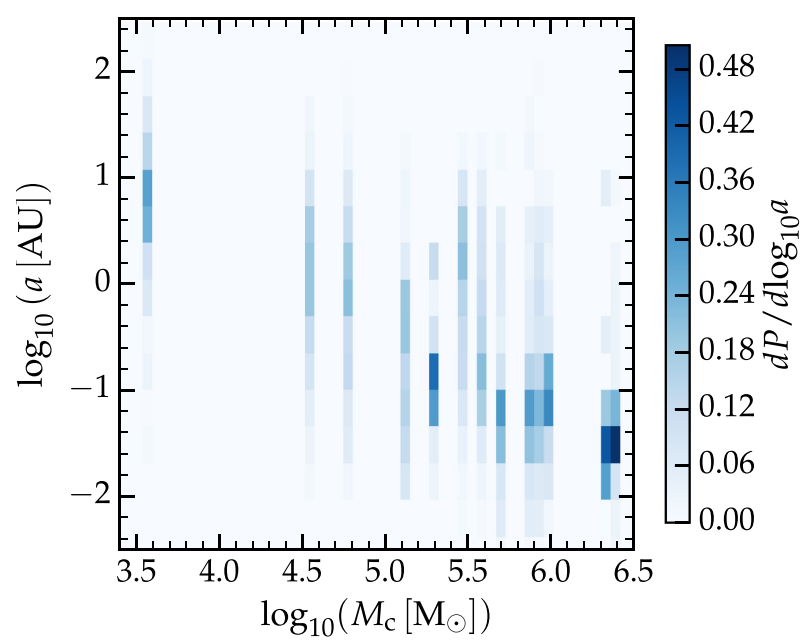

Figure 6. The distributions of the semimajor axes at ejection $a$ as a function of the total cluster mass $M_{\mathrm{c}}$ for the ejected binaries. Each vertical bar represents the distribution of $a$ for the corresponding mass $M_{\mathrm{c}}$ and is normalized such that the integral over $\log _{10} a$ in each mass bin yields unity. High-mass clusters require a high velocity for escape, which a binary must acquire through three-body interactions in order to be ejected. The energy needed to escape is more easily gained once the orbital separation has decreased sufficiently (see equation 41). As a consequence, the mean value of $a$ at ejection shifts to smaller separation with increasing cluster mass $M_{\mathrm{c}}$.

(see Fig. 4 and Table 3). This behaviour can be attributed to the fact that the BHs are not in energy equipartition with the rest of the cluster. Adding more BHs without affecting the distribution of the luminous cluster members requires that the BHs are spread out farther from the core, where they have traditionally been expected to reside. Accordingly, the mean density of BHs goes down, and they are less likely to interact with each other. However, because they are well mixed with the stars at larger radii, the number of BH-NC binaries that form in three-body exchanges grows. Additionally, since these binaries form farther from the core, they also have the benefit of a shallower potential to climb out of.

Besides influencing the number of ejected binaries, the number of retained $\mathrm{BHs}$ also affects the distribution of the semimajor axes of the ejected binaries. In Fig. 7, we show the distribution of semimajor axes for the ejected $\mathrm{BH}-\mathrm{NC}$ binaries in our cluster model for NGC 5694 for the three different choices of BHs retained. We choose this cluster since it is representative of the effect that the number of retained $\mathrm{BHs}$ has on the population of ejected $\mathrm{BH}-\mathrm{NC}$ binaries. Fig. 7 displays an increase in the width of the distribution of semimajor axes for larger populations of BHs. This is again related to the necessary spreading of the BHs, as we increase the number of BHs harboured by the cluster.

Therefore, the $\mathrm{BH}-\mathrm{NC}$ binaries that form outside of the core, where the escape velocity drops rapidly as a function of radius, can be ejected while their binding energies are of comparably lower magnitudes. Although the more widely separated binaries are less likely to become mass-transferring systems, the simulations with large $\mathrm{BH}$ numbers tend to have much higher ejection rates. The higher ejection rates still produce enough tight binaries in the tail of distribution to outnumber those produced with fewer BHs present.

The remaining structural property of GCs that has a clear effect on the population of ejected binaries is the cluster density. In Fig. 8, we plot the distribution of ejection times as a function of the luminous central density, which is related to the core density as discussed in Section 2.2.2. The distribution establishes that the cluster density

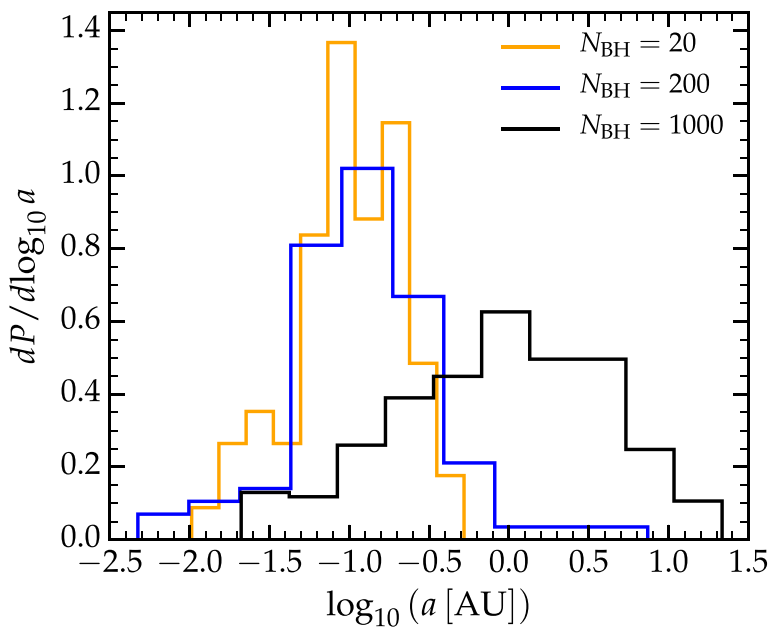

Figure 7. The probability distribution for the ejected BH-NC binary semimajor axes from NGC 5694, a representative case, with a population of 20 , 200, and $1000 \mathrm{BHs}$. An increase in the number of BHs requires spreading the BHs outside of the core, where they are more likely to form binaries with $\mathrm{NC}$ objects. In the outskirts, the energy necessary to escape is much smaller, allowing the binary to escape before it has had sufficient time to harden. These binaries escape with comparatively low-magnitude binding energy and wide orbital separations.

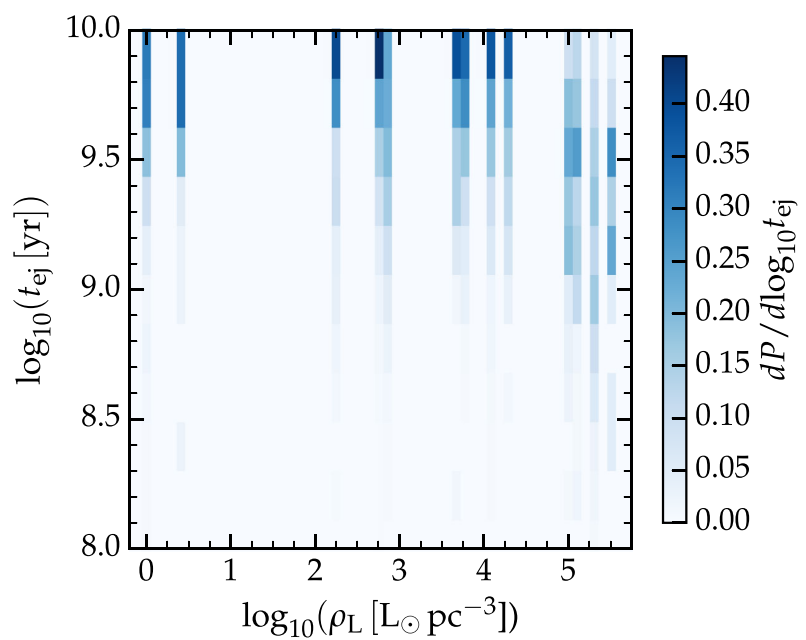

Figure 8. The distributions of time of ejection $t_{\mathrm{ej}}$ as a function of the luminous central density $\rho_{\mathrm{L}}$ for the ejected binaries. Each vertical bar represents the distribution of $t_{\mathrm{ej}}$ for the corresponding core luminosity density $\rho_{\mathrm{L}}$ and is normalized such that the integral over $\log _{10} t_{\mathrm{ej}}$ in each density bin yields unity. In higher-density clusters, where encounters occur more frequently, many binaries are ejected after only a few Gyr, while in the lower-density clusters most ejections occur near the end of the 10 Gyr evolution.

has some impact on the time at which binaries are ejected from their host GCs. The time between binary-single encounters can be approximated by

$t_{\mathrm{enc}}=\Gamma^{-1}=\frac{v_{\mathrm{m}}}{2 \pi G\left(m_{\mathrm{b}}+\bar{m}\right) n_{\mathrm{o}} a}$,

where $v_{\mathrm{m}}$ is the mean velocity of stars in the cluster, $n_{\mathrm{o}}$ is its core density, and $\bar{m}$ is the mean mass. Combining this result with equation (40), we can obtain an approximation for the rate at which a binary increases its kinetic energy $\Delta T / \Delta t$. As encounters approximately 
occur in increments of the encounter time-scale, letting $\Delta t=t_{\text {enc }}$, we find that the rate at which the binary increases its kinetic energy,

$\frac{\Delta T}{\Delta t}=\left(\frac{2 \pi G^{2} m_{1} m_{2} m_{3} \epsilon}{v_{\mathrm{m}}}\right) n_{\mathrm{o}}$,

scales with the cluster core density. Therefore, the time it takes for a binary to acquire a high enough velocity to escape is reduced for higher-density clusters. As can be seen in Fig. 8, in clusters of higher density, where encounters occur more frequently, most $\mathrm{BH}-\mathrm{NC}$ systems are ejected after only $3 \mathrm{Gyr}$ of evolution, whereas in lower-density clusters most ejections take place near the end of the $10 \mathrm{Gyr}$ simulation (i.e. the present day),

\subsection{Black-hole low-mass X-ray binaries}

Here, we focus strictly on the population of the present-day masstransferring systems that have successfully become BH-LMXBs. These results reflect the contribution to the BH-LMXB population from the entire population of non-core-collapsed Milky Way GCs. The production of BH-LMXBs is based on a subset of 15 simulated GCs and the methods detailed in Section 3.2. In the following section, we discuss the distribution and the properties of this population of BH-LMXBs from GCs.

As discussed at the end of Section 3.1, some clusters require choosing a $\mathrm{BH}$ retention fraction of unity, $f_{\mathrm{r}_{\mathrm{BH}}}=1$, in order to obtain the desired quantity of BHs. This occurs in the lowest-mass cluster for each set of $N_{\mathrm{BH}}$, i.e. Pal 13 for $N_{\mathrm{BH}}=20$, NGC 6838 for $N_{\mathrm{BH}}=200$, and NGC 6121 for $N_{\mathrm{BH}}=1000$. These specific parameter sets are not used in determining the population of $\mathrm{BH}-$ LMXBs. Although the results from these three sets are included in the previous discussions, they are excluded here due to the unphysical nature of complete $\mathrm{BH}$ retention. During $\mathrm{BH}$ formation, natal kicks ensure that at least some fraction of the BHs formed from the IMF are ejected from the cluster. This makes complete $\mathrm{BH}$ retention essentially unattainable. In consideration of this, we include only those models with $f_{\mathrm{rBH}}<1$.

\subsubsection{Population}

The number of mass-transferring systems that develops from the $\mathrm{BH}-\mathrm{NC}$ binaries that are ejected from our model clusters strongly depends on the assumed BH retention in GCs. We employ the same notation as in Section 3.2.2 for $\mathrm{BH}$ retention: MIN refers to $N_{\mathrm{BH}}=20,200$ refers to $N_{\mathrm{BH}}=200$, and MAX refers to $N_{\mathrm{BH}}=1000$. The populations are generated from $10^{4}$ realizations in the MIN and 200 case and from $5 \times 10^{3}$ realizations for the MAX case, as described in Section 3.2.2. The resulting BH-LMXB population distributions are presented in Fig. 9. The MIN case produces zero observable BH-LMXB systems. The 200 case produces $25_{-6}^{+10}$ masstransferring $\mathrm{BH}$ low-mass systems and the MAX case yields an expectation value of $156_{-24}^{+26}$ ejected $\mathrm{BH}-\mathrm{LMXBs}$, with the stated uncertainties bounding the 95 per cent confidence interval.

The clusters that contribute the largest number of BH-LMXBs are those with the highest $\mathrm{BH}-\mathrm{NC}$ ejection rates (see Table 3). As is visible in Fig. 4, the expected number of ejections can be approximated as a function of the number of retained $\mathrm{BHs} N_{\mathrm{BH}}$ and the two fundamental parameters describing the cluster: the concentration $c$ and the total cluster mass $M_{\mathrm{c}}$. While the initial semimajor axis at ejection $a$, which is sensitive to the cluster mass (Fig. 6), is an important factor in determining whether a $\mathrm{BH}-\mathrm{NC}$ will lead to mass transfer, surprisingly, the fraction of $\mathrm{BH}-\mathrm{NCs}$ that become

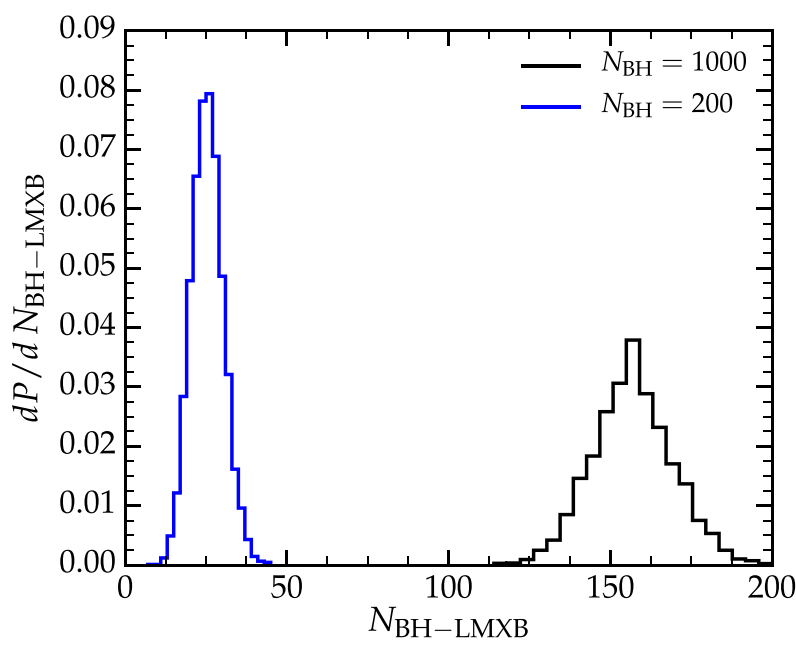

Figure 9. The distribution of the number of BH-LMXBs, $N_{\mathrm{BH}-\mathrm{LMXB}}$, produced from $10^{4}$ realizations for the $N_{\mathrm{BH}}=200$ case and $5 \times 10^{3}$ realizations for the $N_{\mathrm{BH}}=1000$ case. The $N_{\mathrm{BH}}=20$ case produces a population of zero $\mathrm{BH}-\mathrm{LMXBs}$ in $10^{4}$ realizations. The expected values for the two producing cases are $25_{-6}^{+10}$ for $N_{\mathrm{BH}}=200$ and $156_{-24}^{+26}$ for $N_{\mathrm{BH}}=1000$, with the stated uncertainties bounding the 95 per cent confidence interval.

BH-LMXBs appears nearly constant across clusters. Equivalently stated, $\left\langle N_{\mathrm{BH}-\mathrm{LMXB}}\right\rangle \sim f_{\mathrm{LMXB}}\left\langle N_{\mathrm{ej}}\right\rangle$ appears to hold true for the set of clusters modelled, where $f_{\mathrm{LMXB}} \sim 0.25$ represents the fraction of ejected $\mathrm{BH}-\mathrm{NC}$ binaries that evolve into BH-LMXBs. Although the distributions of most orbital parameters, which determine whether a system will evolve into a BH-LMXB, vary from cluster to cluster, the thermal eccentricity distribution shared by all clusters ensures that a roughly equal proportion of the ejected binaries will become $\mathrm{BH}-\mathrm{LMXBs}$. For clusters that tend to eject wider binaries, it is only the highly eccentric systems that become BH-LMXBs, and vice versa.

For a given $\mathrm{BH}$ retention, the number of successfully formed BH-LMXBs from GCs is potentially a function of the ejection time, initial separation, initial eccentricity, primary and companion masses, and the complex internal evolution of the binary. Yet, since we find that the ejection properties are largely determined by the cluster properties, namely the quantities defining the Fundamental Plane, the size of the BH-LMXB population from GCs is well approximated by the cluster properties alone.

\subsubsection{Distribution}

As GCs generally have low escape velocities, the ejected $\mathrm{BH}$ LMXBs typically escape with relatively low velocities. Due to this, the distribution of BH-LMXBs closely mimics the distribution of GCs in the Milky Way galaxy. In Fig. 10, we present the spatial probability distribution of BH-LMXBs from GCs, for the MAX case, on a Mollweide projection of the Galactic map in longitudinal and latitudinal Galactic coordinates $(l, b)$. Additionally, we include the distribution of Galactic GCs and known BHLMXBs from BlackCAT (Corral-Santana et al. 2016), a catalogue of candidate $\mathrm{BH}-\mathrm{LMXBs}$, which we use in all figures including an observed population, unless stated otherwise. Although the 200 case produces fewer BH-LMXBs, the distribution is qualitatively similar to the MAX case. The highest probability density region is near the Galactic centre, where the majority of GCs reside. However, as Fig. 5 illustrates, the distributions of the ejection velocities 


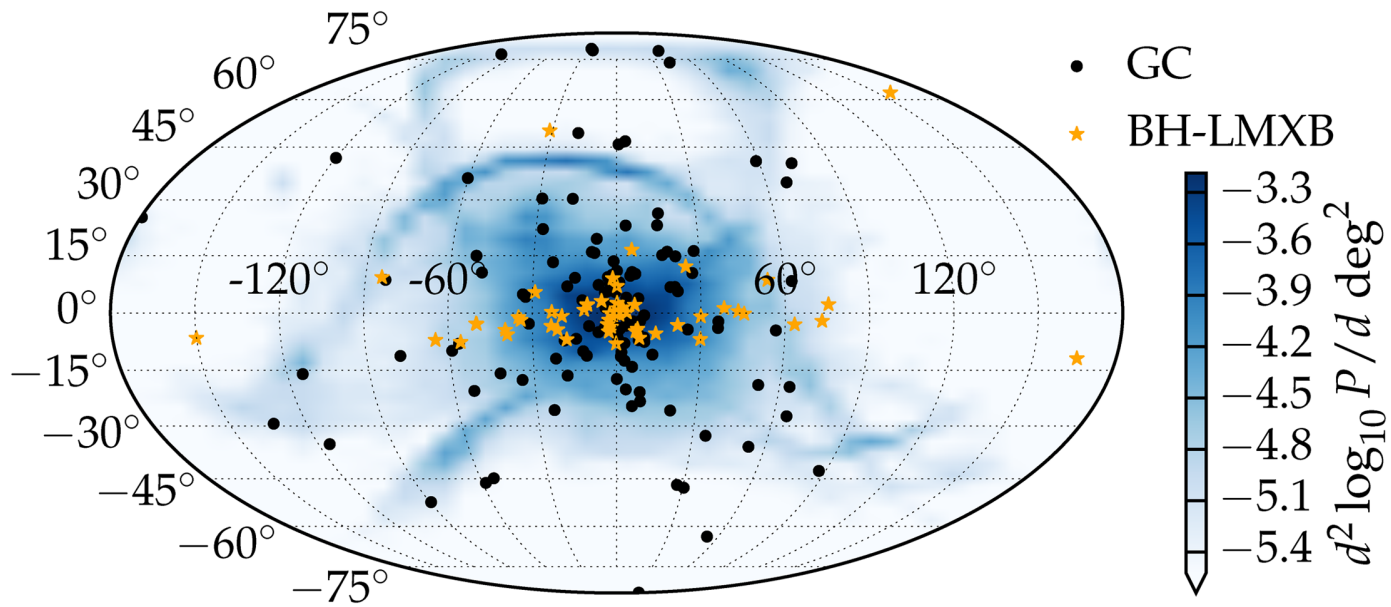

Figure 10. The spatial probability distribution of the simulated population of BH-LMXBs from GCs with $N_{\mathrm{BH}}=1000$. The populations of Milky Way GCs (marked by black circles) and known BH-LMXBs (marked by orange stars) are included for reference. The map is a Mollweide projection of the $(l, b)$ Galactic coordinate system. The Galactic centre is located near $0^{\circ}$ latitude and $0^{\circ}$ longitude, where the high density of objects explains the clustering of BH-LMXBs and GCs.

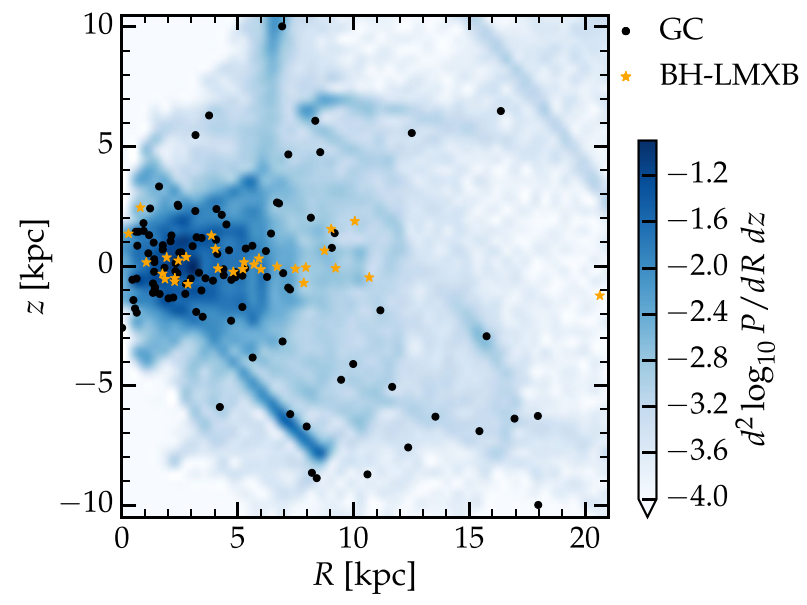

Figure 11. The spatial probability distribution of the simulated population of BH-LMXBs from GCs with $N_{\mathrm{BH}}=1000$ in the $R-z$ plane. The coordinate $z$ specifies the distance perpendicular to the Galactic plane and $R$ is the inplane distance from the Galactic centre at the origin. The populations of Milky Way GCs (marked by black circles) and known BH-LMXBs (marked by orange stars) are included for reference. While many of the BH-LMXBs from GCs populate the Galactic disc, the distribution extends well out of the Galactic plane into the high- $|z|$ region.

have widths that span an order of magnitude or more. As a consequence, some fraction of the binaries have ejection velocities that allow them to separate from their parent cluster. Additionally, the binaries that are ejected at an earlier time in the GC's orbit have sufficient time to diverge from the host GC orbit. The higher-density streaks in Fig. 10 can be attributed to these binaries that have drifted from the parent GC.

As GCs primarily follow halo orbits that extend well out of the Galactic plane, the GCs are easily able to populate this space with BH-LMXBs. In Fig. 11, we provide the spatial probability distribution for $\mathrm{BH}-\mathrm{LMXB}$ from the MAX case in the $R-z$ plane.

Again, we present only the MAX case, as the 200 case is similarly distributed but with a lower overall probability density. The median absolute distance from the Galactic plane is $|z|=1.63 \mathrm{kpc}$

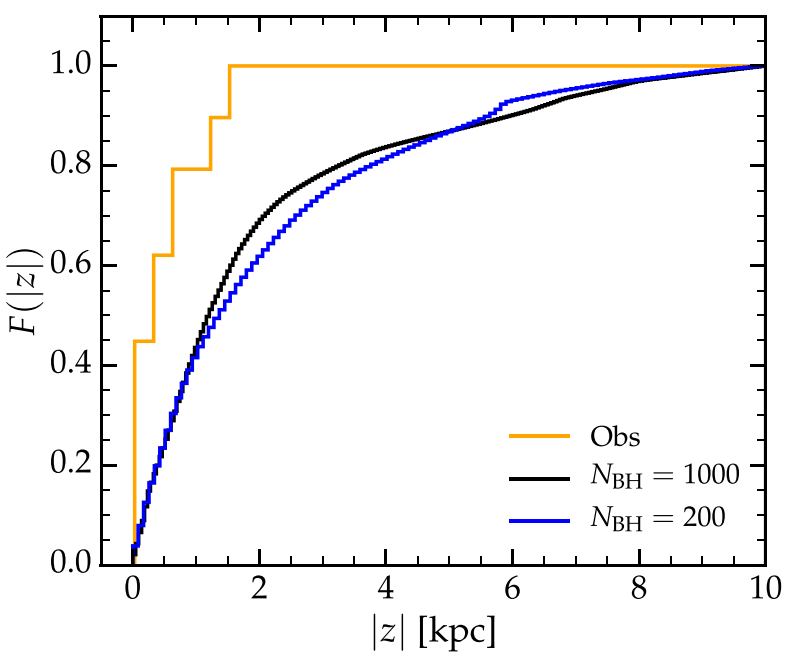

Figure 12. The normalized $\mathrm{CDF}$ of the absolute distance perpendicular to the Galactic plane $|z|$. The included distributions are the BH-LMXBs produced in our GC simulations for the cases of $N_{\mathrm{BH}}=200, N_{\mathrm{BH}}=1000$, and the observed population. Note that in the case that GCs have minimal $\mathrm{BH}$ retention $\left(N_{\mathrm{BH}}=20\right)$, no mass-transferring systems are produced.

and the median distance from the Galactic centre in the plane is $R=4.51 \mathrm{kpc}$. While it is clear from Fig. 11 that many of the $\mathrm{BH}-\mathrm{LMXBs}$ from GCs are located in the Galactic disc, the distribution extends well out of the Galactic plane into the lower-density regions above and below the disc. BH-LMXBs that form in the field will generally reside in the high-density Galactic plane, unless they receive substantial kicks at birth, which might eject them into the 'high-z' regions. However, the magnitude of BH-LMXB kicks is still uncertain and the magnitude necessary to reach the highest of BH-LMXBs from GCs is considered unlikely (see e.g. Repetto \& Nelemans 2015; Mandel 2016). In Fig. 12, we show the CDF of the absolute distance $|z|$ perpendicular to the Galactic plane for the MAX case, the 200 case, and the observed population of BH-LMXBs. The observed population terminates at a maximum $|z| \sim 2 \mathrm{kpc}$, while the BH-LMXB population from GCs extends well beyond this point. This produces a region of space that is unique to 


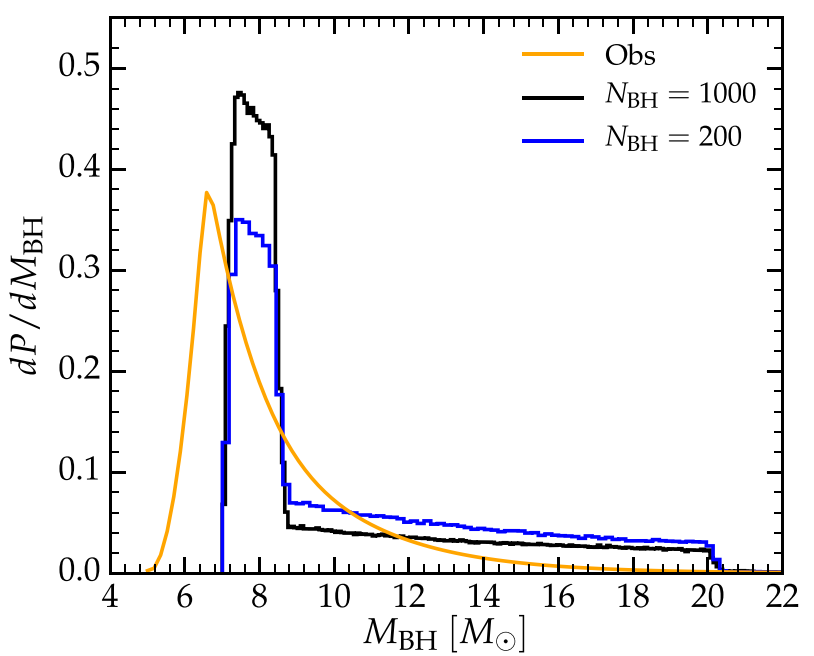

Figure 13. The probability distributions of $\mathrm{BH}$ masses in BH-LMXBs for the observed population (Özel et al. 2010) and for the BH-LMXBs produced in our GC simulations for the cases of $N_{\mathrm{BH}}=200,1000$. Note that in the case that GCs have minimal BH retention $\left(N_{\mathrm{BH}}=20\right)$, no mass-transferring systems are produced. The discontinuous jumps in the distribution correspond to the mass bin minimum and maximum, with a power-law distribution inbetween determined by the EMF. The lowest-BH-mass bin was truncated at $7 \mathrm{M}_{\odot}$.

a population of $\mathrm{BH}-\mathrm{LMXBs}$ from GCs, a population distinct from those forming in the field.

\subsubsection{Properties}

A typical BH-LMXB with a GC origin has an initial semimajor axis of $5.71 R_{\odot}$, initial $\mathrm{BH}$ mass of $8.09 \mathrm{M}_{\odot}$, and an initial companion mass of $0.4 \mathrm{M}_{\odot}$. The median present-day period is $4.48 \mathrm{~h}$ and the median present-day $\mathrm{BH}$ mass is $8.25 \mathrm{M}_{\odot}$, which has increased above the initial median $\mathrm{BH}$ mass due to accretion from the companion. As discussed in Section 3.2.2, the masses used in the Monte Carlo models for the ejected binaries are sampled according to the EMF from the mass bin corresponding to the mass in the ejected $\mathrm{BH}-\mathrm{NC}$. This is done for both the primary $\mathrm{BH}$ mass $M_{\mathrm{BH}}$ and the companion mass $m_{2}$ to obtain the mass distributions, which we discuss below.

In Fig. 13, we show the distribution of the BH mass in the population of $\mathrm{BH}-\mathrm{LMXBs}$ from $\mathrm{GCs}$ for both cases that produce masstransferring systems.

Along with the $\mathrm{BH}$ mass distributions for the 200 and MAX cases, we include the inferred $\mathrm{BH}$ mass distribution from observations (Özel et al. 2010). Although the observed mass distribution reaches down to $\sim 5 \mathrm{M}_{\odot}$, our EMF does not produce $\mathrm{BH}$ masses in the range $M_{\mathrm{BH}}<7 \mathrm{M}_{\odot}$. The $\mathrm{BH}$ primary mass is peaked at $7.4 \mathrm{M}_{\odot}$ and displays a preference for the lower-mass BHs. The lack of systems at high-mass $\mathrm{BH}$ can be attributed to two contributing factors. The leading contribution is the distribution of $\mathrm{BH}$ masses in the ejected $\mathrm{BH}-\mathrm{NCs}$, which is dominated by the two lowest-BH-mass bins (i.e. $8.87 \mathrm{M}_{\odot}$ and $\left.20.48 \mathrm{M}_{\odot}\right)$. Although these are produced in nearly equal numbers, the preference for the lowest-mass bin that arises in the BH-LMXBs is due to a secondary effect introduced during the binary stellar evolution. High-mass ratio systems are prone to disrupting the companion star, ending the possibility of evolving into a stable BH-LMXB. Despite these barriers to forming $\mathrm{BH}-$ LMXBs with high-mass BHs, there remains a small population of

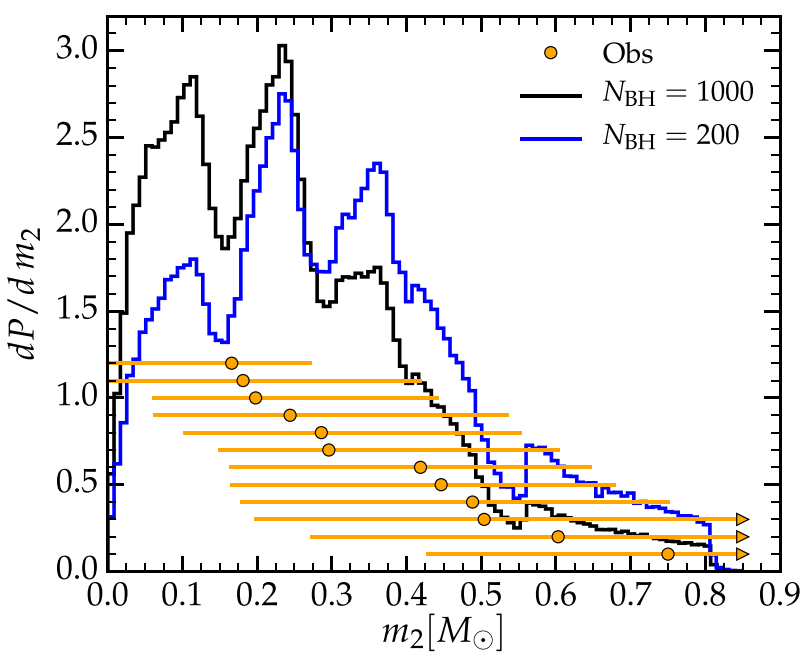

Figure 14. The probability distributions of the companion masses in $\mathrm{BH}$ LMXBs for the cases $N_{\mathrm{BH}}=1000$ and $N_{\mathrm{BH}}=200$. The observed population includes 12 of the 18 confirmed BH-LMXBs in BlackCAT (CorralSantana et al. 2016) that have the necessary observational quantities (see Section 4.2.3 for a description of the observed population) and are included for reference; the circles indicate the mean value, the line represents the uncertainty in the observations, and the inclusion of an arrow indicates that the uncertainty is only bounded on one side. The remaining 6 confirmed $\mathrm{BH}-$ LMXBs have companion masses above the range considered here, where the axis has been truncated to focus on the range of masses less than the MS turn-off mass $m_{\text {to }}=0.85 \mathrm{M}_{\odot}$. The peaks in the simulated distributions are due to the sampling of companion masses from the EMF within each mass bin.

high-mass present-day BH-LMXBs, with $M_{\mathrm{BH}}>40 \mathrm{M}_{\odot}$, which accounts for $\sim 1$ per cent of the population.

The low-mass companions are restricted to the range $m_{2}<0.85 \mathrm{M}_{\odot}$, where the maximum mass is constrained by the MS turn-off mass, $m_{\text {to }}=0.85 \mathrm{M}_{\odot}$. The present-day companion mass is a function of the mass-transfer rate and the time since the onset of mass transfer. The majority of the companion masses are MS stars; however, there exists a sub-population of WD companion masses that account for $\sim 10$ per cent of the companions in the MAX case and $\sim 20$ per cent in the 200 case. In Fig. 14, we display the companion mass distribution for the MAX case, 200 case, and the observed population of BH-LMXBs.

The lack of lower-mass companions in the 200 case relative to the MAX case is due to the higher fraction of WDs, which have masses $m_{\mathrm{WD}} \gtrsim 0.4 \mathrm{M}_{\odot}$. In the MAX case, there is a larger number of BHs in the outskirts where the lowest masses reside, whereas the 200 case is more centrally concentrated where there is an increase in the probability of picking up a higher-mass companion and which includes a larger population of WDs. The observed population in Fig. 14 is generated from the observational data in the candidate $\mathrm{BH}-$ LMXB catalogue BlackCAT. There are 18 confirmed BH-LMXBs in the catalogue that have a measurement of the $\mathrm{BH}$ mass $M_{\mathrm{BH}}$ and the mass ratio $q$, which we use to estimate the companion mass $m_{2}=q M_{\mathrm{BH}}$. The companion masses in the observed population have large error bars due to the uncertainty in the measurements of the $\mathrm{BH}$ mass and the mass ratio.

The initial eccentricity of the binaries follows a thermal distribution, while the initial semimajor axis, as discussed in 4.2 .1 , is typically $(a / \mathrm{AU}) \ll 1$, due to their GC origin. The small initial separation of the BH-NCs leads to a distribution of periods $p$ where $\sim 99$ per cent of the BH-LMXBs have $p \lesssim 6.2 \mathrm{~h}$ for the MAX case 


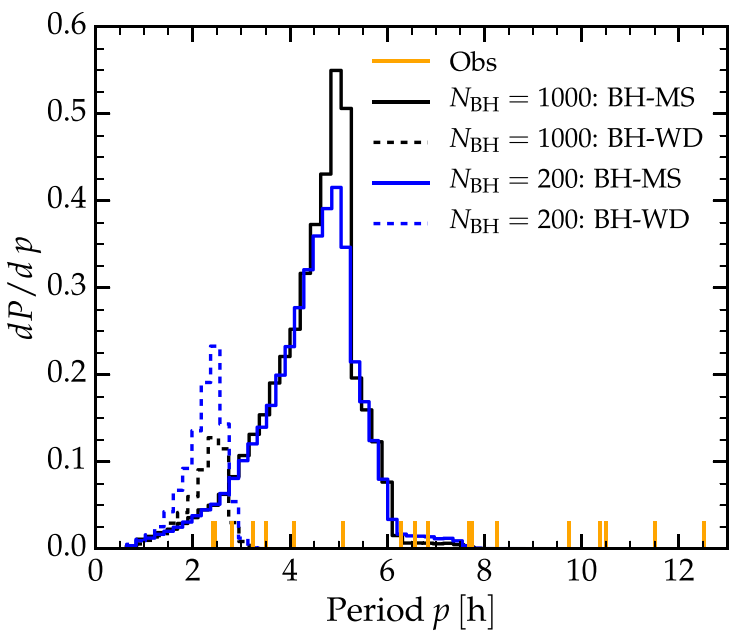

Figure 15. The probability distribution of orbital periods in the simulated BH-LMXBs from GCs for the two stellar companion sub-populations: WD and MS. The periods for the observed population of BH-LMXBs that are less than $13 \mathrm{~h}$ are included for reference and are identified by orange tick marks ( 18 of the 28 candidate BH-LMXBs from BlackCAT). To preserve the relative size of the MS and WD companion populations, each distribution is independently normalized and then multiplied by the factors $N_{\mathrm{BH}-\mathrm{MS}} / N$ and $N_{\mathrm{BH}-\mathrm{WD}} / N$, respectively, with $N=N_{\mathrm{BH}-\mathrm{MS}}+N_{\mathrm{BH}-W D}$. This normalization is applied to each $N_{\mathrm{BH}}$ case independently.

and $p \lesssim 6.8 \mathrm{~h}$ in the 200 case. The sub-population of BH-LMXBs with a WD companion have a qualitatively similar distribution but with a reduced period such that $\sim 99$ per cent of the population have $p \lesssim 3 \mathrm{~h}$ for both cases, MAX and 200. The reduced period for the WD companions is due to the smaller separations necessary to induce mass transfer for these compact objects. In Fig. 15, we display the bi-modal distribution of the orbital period for our population of BH-LMXBs along with a subset of the observed population with periods less than $\sim 1 / 2 \mathrm{~d}$.

The mass transfer in these systems is primarily driven by angular momentum loss due to tidal circularization. As the companion star passes the $\mathrm{BH}$ at periastron, the tidal forces from the $\mathrm{BH}$ deform the star and dissipate energy. This tidal torque efficiently removes eccentricity from the system and eventually leads to circularization of the orbit with a reduced period. Once the period reaches some critical separation, the companion star overfills its Roche lobe and transitions to a state of mass transfer. This is the same mechanism operating on the BH-LMXBs with a WD companion; however due to the compact nature of WDs, the critical separation that leads to Roche lobe overflow occurs at smaller separations, hence the shorter orbital periods. The binary evolution for the BH-LMXBs from GCs is significantly different from the evolution of field binaries. In the standard binary evolution picture, the companion evolves to overfill its Roche lobe, which can lead to mass transfer at relatively large separations. The MS stars in BH-LMXBs from GCs have not evolved significantly within the cluster, but evolve on much longer time-scales, preventing them from achieving mass transfer at wide separations.

In Fig. 16, we provide a temperature-luminosity diagram for the mass-transferring MS companions. We exclude the WD systems from the diagram, since they are likely too faint for observation. The MS companions have temperatures $\sim 1500-6300 \mathrm{~K}$ and luminosities $\sim 6 \times 10^{-4}-0.5 \mathrm{~L} \odot$, making these identifiable as $\mathrm{K} / \mathrm{M}$ late-type MS stars below the MS turn-off.

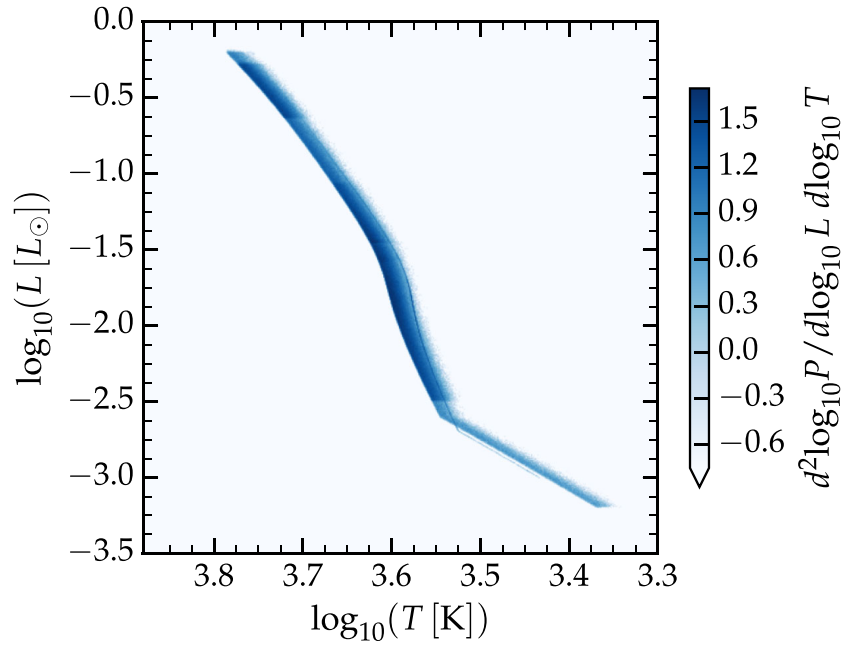

Figure 16. Temperature-luminosity diagram for the BH-LMXB companion mass in the simulated population of BH-LMXBs from GCs with $N_{\mathrm{BH}}=1000$. The low-luminosity WD companions are excluded from the figure, leaving only the mass-transferring MS companions. Since the MS companions from GCs are unevolved stars, the companion temperatureluminosity diagram is essentially the portion of the Hertzsprung-Russell MS branch with $m_{2}<m_{\text {to }}$.

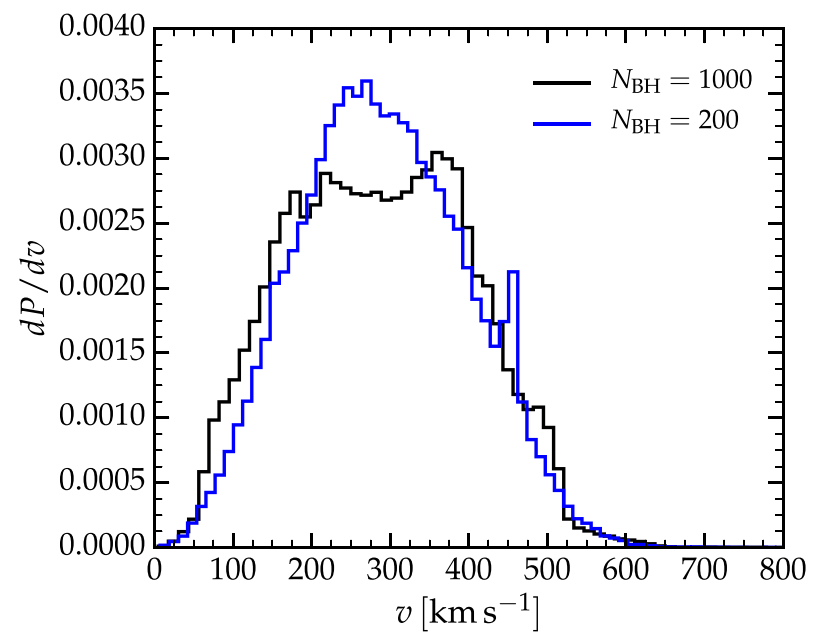

Figure 17. The probability distributions for the space velocity $v$ of the simulated BH-LMXB population for the two $\mathrm{BH}$ s retention values $N_{\mathrm{BH}}=1000$ and $N_{\mathrm{BH}}=200$. The BH-LMXB space velocity is $\boldsymbol{v}=\boldsymbol{v}_{\mathrm{ej}}+\boldsymbol{v}_{\mathrm{GC}}$, where $\boldsymbol{v}_{\mathrm{ej}}$ is the ejection velocity and $\boldsymbol{v}_{\mathrm{GC}}$ is the velocity of the host GC. Since $\boldsymbol{v}_{\mathrm{ej}}$ is approximately the GC escape velocity, the magnitude $v$ is dominated by the relatively large contribution from $\boldsymbol{v}_{\mathrm{GC}}$. As such, the velocity distribution of BH-LMXBs is consistent with the velocity distribution of GCs, which is reflected in the high mean velocities.

A distinct characteristic of these systems are their kinematic properties. In Fig. 17, we show the distribution of the magnitude of the velocity $v$ of the BH-LMXBs from GCs.

The velocity $v$ is computed from the components of the space velocity in the heliocentric Galactic coordinate system $(U, V, W)$, a right-handed coordinate system with $U$ in the direction of the Galactic centre, $V$ along the direction of rotation, and $W$ pointing towards the Galactic north pole. The median values of the velocity components for the MAX case are $(U, V, W)=(-24.47,-211.31$, $-22.23) \mathrm{km} \mathrm{s}^{-1}$. The large negative velocity in the $V$ component is indicative of this population not participating in Galactic rotation. 
The peculiar velocity - the velocity of a source relative to a local standard of rest, obtained by removing the contribution of Galactic rotation at the source distance in the Galactic plane $R$ - is sometimes used to infer a 'natal kick' for BH-LMXBs. Although it is possible to convert the Galactic space velocity to a peculiar velocity, this inferred 'kick velocity' is only justified in assuming the source was born in the Galactic disc, where it participates in Galactic rotation. For BH-LMXBs formed in the field, which is most likely to occur in the disc, this is a reasonable assumption. However, the $V$ component of the BH-LMXBs from GCs indicate low rotational velocities, which is consistent with the parent GC halo orbits, which are typically non-circular and extend well out of the Galactic plane. As the BH-LMXBs with GC origins are ejected at relatively low velocities along the GC's orbit in the Galaxy, this population of BHLMXBs has a velocity distribution consistent with the high-velocity halo orbits of GCs. As these systems have high apparent peculiar velocities, due to their halo orbits and the lack of participation in Galactic rotation, attempting to infer a 'natal kick' from the peculiar velocity in such a case is ill-posed and leads to the conclusion of a large required 'natal kick.'

\subsection{Merger events}

\subsubsection{GW-driven mergers}

As briefly discussed in Section 2.3, we allow for gravitationalradiation-driven mergers between compact objects. Since all of our 'test binaries' contain at least one $\mathrm{BH}$, the allowable set of $\mathrm{GW}$ merger pairs is limited to BH-NS, BH-WD, and BH-BH. In addition to those binaries that merge during their evolution within the cluster, binaries of these types can also be ejected from the cluster. In the case of the ejection of a compact pair, we calculate the expected merger time $t_{\mathrm{d}}$ using the ejected binary parameters and refer to these as post-ejection mergers if $t_{\mathrm{ej}}+t_{\mathrm{d}}<t_{\mathrm{H}}$, where $t_{\mathrm{H}}=10^{10} \mathrm{yr}$ is approximately the Hubble time. The total merger rate includes these post-ejection mergers in addition to the in-cluster mergers. Here, we present an estimate of the merger rates averaged over the $10^{10} \mathrm{yr}$ simulations for different $\mathrm{BH}$ retention values.

For notational convenience, we refer to a parameter set as $x_{i}$, where the index $i$ runs over the 39 parameter sets that make up each row of Table 2 and corresponds to a specific GC and value of $N_{\mathrm{BH}}$. We compute the expected number of mergers for each parameter set by considering the probability of a $\mathrm{BH}$ being involved in a merger, defined simply by $P_{\mathrm{m}}\left(x_{i}\right)=\frac{N_{\text {mergers }}\left(x_{i}\right)}{N_{\mathrm{runs}}\left(x_{i}\right)}$, multiplied by the BH population

$\left\langle N_{\mathrm{m}}\right\rangle_{i}=P_{\mathrm{m}}\left(x_{i}\right) N_{\mathrm{BH}}\left(x_{i}\right)$.

In the case of a merger involving two BHs, the expectation value is calculated using $N_{\mathrm{BH}}\left(x_{i}\right) / 2$ in order to avoid double counting. The rightmost three columns of Table 4 list the expected number of GWdriven compact object mergers over the lifetime of each cluster for a given $\mathrm{BH}$ population. The number of $\mathrm{BH}-\mathrm{BH}$ mergers is strongly correlated with the GC core density $n_{\mathrm{o}}$. Each population of $\mathrm{BHs}$ has a merger expectation value that follows a power law in the core density with exponent $\sim 0.58$. Since we do not include primordial binaries, exchange encounters are the only means to forming $\mathrm{BH}-$ $\mathrm{BH}$ binaries that can later merge. The average rate of encounters is directly proportional to the density, with the highest-density clusters providing the largest number of opportunities to successfully form $\mathrm{BH}-\mathrm{BH}$ binaries. There are additional correlated variables, such as the concentration $c$ and velocity dispersion $\sigma$; however, these are secondary to the density $n_{\mathrm{o}}$ and likely due to their own correlation with $n_{\mathrm{o}}$.

Given the expected number of mergers for each cluster, we determine a weighted average using the GC mass function, since the total cluster mass of GCs is not uniformly distributed (McLaughlin \& Pudritz 1996). We do this individually for each group of simulations belonging to the sets $N_{\mathrm{BH}}=\{20,200,1000\}$, utilizing the GC mass spectrum $\mathrm{d} N\left(M_{\mathrm{c}}\right) / \mathrm{d} M_{\mathrm{c}}$ of McLaughlin \& Pudritz (1996). For each simulated cluster, we assign a weight $w_{i}=N\left(M_{\mathrm{c}}\left(x_{i}\right)\right)$ and compute the expected number of mergers per cluster in the Milky Way from

$\left\langle N_{\mathrm{m}}\left(N_{\mathrm{BH}}\right)\right\rangle=\frac{\sum_{i} w_{i}\left\langle N_{\mathrm{m}}\right\rangle_{i}}{\sum_{i} w_{i}}$.

For clarity, to obtain the expected number of mergers for $N_{\mathrm{BH}}=20$, we sum over all parameter sets in Table 2 with $N_{\mathrm{BH}}=20$. The resulting expected number of $\mathrm{BH}-\mathrm{BH}$ mergers over the life of a cluster for each choice of $N_{\mathrm{BH}}$ are $\left\langle N_{\mathrm{m}}(20)\right\rangle=0.513,\left\langle N_{\mathrm{m}}(200)\right\rangle=5.08$, and $\left\langle N_{\mathrm{m}}(1000)\right\rangle=62.5$.

We convert the expected number of mergers to a merger rate density by assuming that our simulations of Milky Way GCs are a fair representation of GCs in other galaxies, that the GCs are all approximately $t_{\mathrm{GC}}=10^{10} \mathrm{yr}$ old, and that the spatial density of GCs in the Universe is $\rho_{\mathrm{GC}}=0.77 \mathrm{Mpc}^{-3}$ (see supplemental materials of Rodriguez et al. 2015). Using the weighted averages computed above as our 'typical' cluster merger values and assigning this value to each GC in the volume, we obtain the merger rate density due to all GCs in the Universe,

$\left\langle R\left(N_{\mathrm{BH}}\right)\right\rangle=\frac{\left\langle N_{\mathrm{m}}\left(N_{\mathrm{BH}}\right)\right\rangle}{t_{\mathrm{GC}}} \rho_{\mathrm{GC}}$.

In Table 5, we provide the computed estimated merger rate densities for compact object mergers due to GCs for the three populations of $N_{\mathrm{BH}}$ we consider. Although there is an increased interest in the $\mathrm{BH}$-mass spectrum for $\mathrm{BH}-\mathrm{BH}$ mergers in GCs, stimulated by the larger than expected $\mathrm{BH}$ masses recently detected by aLIGO (Abbott et al. 2016d), the use of just three discrete $\mathrm{BH}$ masses precludes the possibility of such an analysis.

Since BH-BH mergers from GCs only partially contribute to the total merger rate, with the remaining mergers coming from the field, the rates due to GCs should not exceed the upper bound of the total estimated merger rate. The most recent observational evidence constrains the $\mathrm{BH}-\mathrm{BH}$ merger rate density to lie in the range of 12-213 $\mathrm{Gpc}^{-3} \mathrm{yr}^{-1}$ (Abbott et al. 2017). The GC BH-BH merger rate densities given in Table 5 for the three different $\mathrm{BH}$ retention scenarios are well below the upper bound, presenting no conflict with the observed rate. It is tempting to rule out the lower $\mathrm{BH}$ retention cases based on their relatively low merger rate densities compared to the observed lower bound. However, we emphasize that the rates presented in Table 5 are the expected rates due to GCs alone, while the observed rate provides bounds on the total $\mathrm{BH}-\mathrm{BH}$ merger rate that includes the contribution from the field. We could attempt to convert the cluster merger rate to a total rate, but this relies on a well constrained value of the GC fractional contribution. Given the large uncertainty in this fractional contribution, any attempt to approximate the total rate will be dominated by the error in the fractional estimate. Therefore, we presently refrain from ruling out certain $\mathrm{BH}$ populations based on their $\mathrm{BH}-\mathrm{BH}$ merger rate densities alone.

The bounds of our merger rates, which span a wide range of uncertainty in $\mathrm{BH}$ retention, are consistent with previous studies that provide estimates of the $\mathrm{BH}-\mathrm{BH}$ merger rate from GCs (O'Leary 
Table 4. Expected number of mergers. For each cluster and number of retained BHs, we list the exact number of BHs in the cluster along with the expected number of mergers over the cluster lifetime. The number of expected mergers within a cluster are Poisson distributed. Denoting each expectation value $\lambda$, the standard deviation, $\sigma$, associated with each value in the table follows from Poisson statistics and is given by $\sigma=\sqrt{\lambda}$.

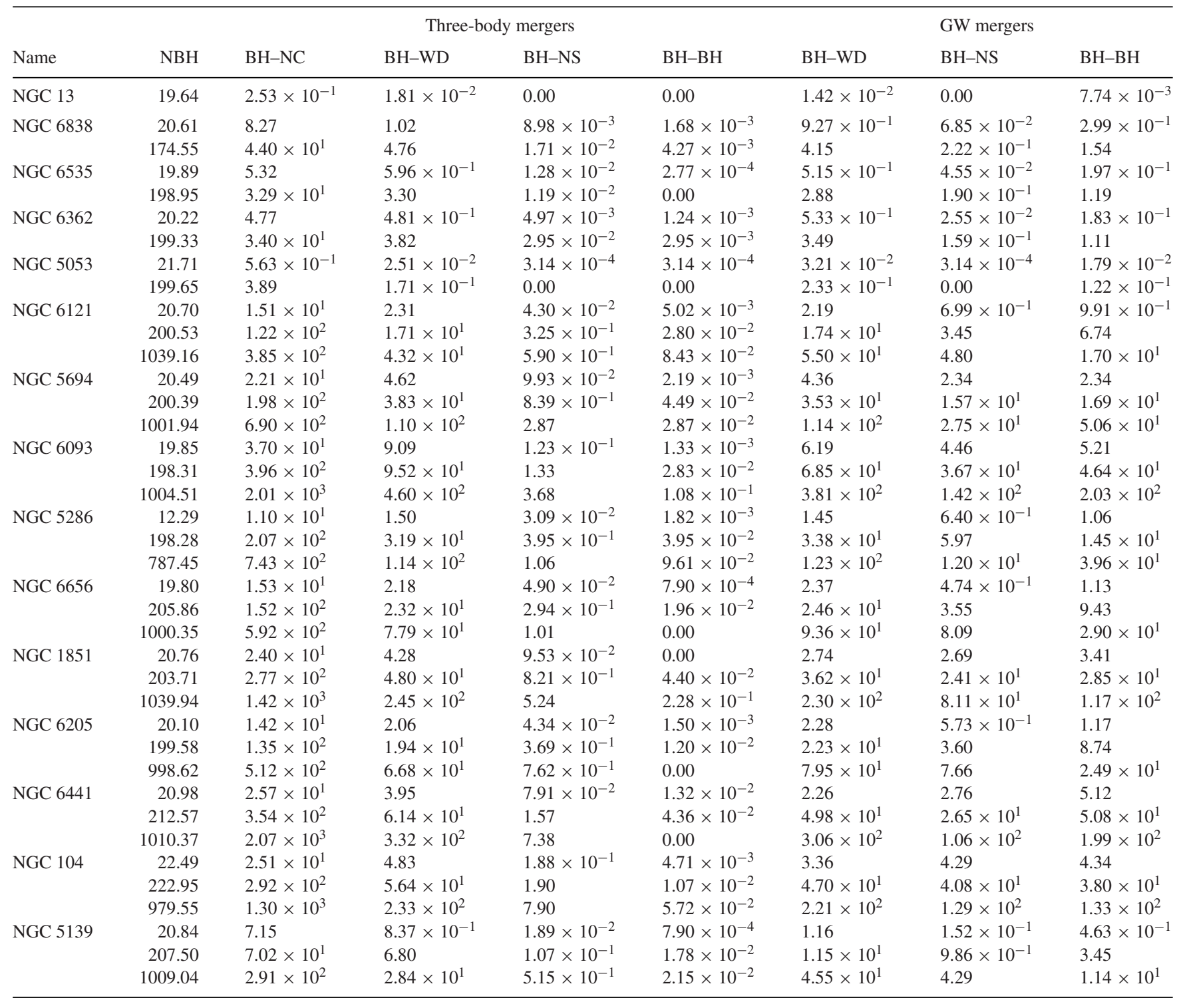

Table 5. The contribution to the compact merger rate density from all GCs in the Universe, stated in $\mathrm{Gpc}^{-3} \mathrm{yr}^{-1}$. Each row corresponds to the merger rate contribution from GCs with the simulated $\mathrm{BH}$ population specified by $N_{\mathrm{BH}}$ in $\left\langle R\left(N_{\mathrm{BH}}\right)\right\rangle$. The merger rate densities are averaged over the life of the cluster, weighted by the GC mass function to account for the nonuniform mass distribution of GCs, and assumes a GC spatial density of $\rho_{\mathrm{GC}}=0.77 \mathrm{Mpc}^{-3}$.

\begin{tabular}{llll}
\hline$\left\langle R\left(N_{\mathrm{BH}}\right)\right\rangle$ & $\mathrm{BH}-\mathrm{BH}$ & $\mathrm{BH}-\mathrm{NS}$ & $\mathrm{BH}-\mathrm{WD}$ \\
\hline$\langle R(20)\rangle$ & $3.95 \times 10^{-2}$ & $2.71 \times 10^{-2}$ & $7.15 \times 10^{-2}$ \\
$\langle R(200)\rangle$ & $3.91 \times 10^{-1}$ & $2.51 \times 10^{-1}$ & $7.73 \times 10^{-1}$ \\
$\langle R(1000)\rangle$ & 4.81 & 2.83 & 10.59 \\
\hline
\end{tabular}

et al. 2006; Sadowski et al. 2008; Downing et al. 2011; Morscher et al. 2015; Rodriguez, Chatterjee \& Rasio 2016a). However, we find that only $\sim 10$ per cent of the BH-BH mergers occur outside of the cluster boundaries, which differs from a subset of these previous studies. In Downing et al. (2011), no mergers occur in cluster, while in Morscher et al. (2015), 85 per cent of BH-BH mergers occur post-ejection, and Rodriguez et al. (2016a) find that $\sim 90$ per cent merge outside the cluster. In contrast to the small number of $\mathrm{BH}-$ BH binaries, these studies find merging in cluster, O'Leary et al. (2006) finds that only 24-72 per cent of the BH-BH mergers are post-ejection. Finally, Sadowski et al. (2008) is most closely aligned with our results, with $\sim 10$ per cent of mergers occurring out of the cluster.

This discrepancy in merger location can be attributed to the distribution of the BHs in the cluster and their interactions with the lower-mass components. In models with centrally clustered BHs, the BHs are segregated from the remainder of the cluster, forming an isolated and decoupled system. These self-interacting BHs efficiently form BH binaries. Strong binary-binary interactions can eject these binary BHs from the cluster, where they might later merge in isolation. In addition to the efficient removal of $\mathrm{BH}$ binaries from the core, binary-single interactions are equally efficient at ejecting single BHs from the cluster. Furthermore, these strong 
encounters are likely to interrupt potential mergers of eccentric $\mathrm{BH}$ binaries that would merge in-cluster if uninterrupted. This channel leads to a majority of $\mathrm{BH}-\mathrm{BH}$ mergers outside of the cluster and eventually depletes the GC of BHs (e.g. O'Leary et al. 2006; Banerjee, Baumgardt \& Kroupa 2010; Downing et al. 2011). We assume that in order for GCs to retain significant BH populations, the BHs must avoid segregating in the core, which we accomplish through a modified velocity dispersion for the BHs, as discussed in Section 2.2.4. This modified velocity dispersion spreads the BHs throughout the cluster, where they can interact with the lower-mass stars. This supposition is similar to the assumptions made in Sadowski et al. (2008) and produces qualitatively similar results.

In our simulations, a key channel for producing $\mathrm{BH}-\mathrm{BH}$ binaries is through the formation of a binary composed of a $\mathrm{BH}$ and a non$\mathrm{BH}$ outside of the core, which eventually drift to the centre where there is a high density of BHs. The non-BH will be preferentially exchanged with one of the more massive $\mathrm{BH}$ in the core, producing a BH-BH binary that will realize one of three outcomes: (1) the BH$\mathrm{BH}$ binary will be dismantled in the high-density region, (2) given a sufficiently large eccentricity (hence a shorter orbital decay time), will eventually merge in the core, or (3) will harden and be ejected from the cluster. This formation channel is similar to that described in Sadowski et al. (2008). As discussed in Section 2.3.5, we allow for single BHs to exchange into existing binaries. The majority of binaries that a single $\mathrm{BH}$ encounters are binaries composed of two low-mass stars. Successful exchanges of a more massive $\mathrm{BH}$ for one of the lower-mass stars tend to produce high-eccentricity $\mathrm{BH}-$ non-BH binaries following the relation

$\langle e\rangle \approx 1-1.3\left(\frac{m_{\text {non-BH }}}{m_{\mathrm{BH}}}\right)$,

which is independent of the initial eccentricity and applicable when $m_{\text {non-BH }} \ll m_{\mathrm{BH}}$ (Sigurdsson \& Phinney 1993). For the three BH masses considered, $M_{\mathrm{BH}}=\{8.87,20.48,57.18\} \mathrm{M}_{\odot}$, and a cluster non-BH star with an average mass of $\left\langle m_{\text {non-BH }}\right\rangle \approx 0.3 \mathrm{M}_{\odot}$, this leads to mean initial eccentricities of $\langle e\rangle \approx\{0.956,0.981,0.993\}$. Once the binary makes it to the core, the non- $\mathrm{BH}$ is easily exchanged for one of the many massive $\mathrm{BHs}$, yielding a highly eccentric $\mathrm{BH}-$ $\mathrm{BH}$ binary according to equation (48). In Fig. 18, we display the eccentricity distributions for the $\mathrm{BH}-\mathrm{BH}$ binaries at formation and at merger or ejection for those binaries that have end states (2) and (3), as described above, respectively. Some fraction of the eccentric binaries that form through this channel are driven to high enough eccentricities that they can merge in-cluster in-between encounters. The remainder are subject to further encounters that drive their eccentricities towards a thermalized distribution, are hardened in the process, and are eventually ejected.

The eccentricity distribution of merging BH-BH binaries is important for the detection of the resulting gravitational waves. The eccentricity tends to zero as the orbit shrinks; however modern detectors are sensitive to the GW signal at frequencies when the binary is still in the inspiral phase and the eccentricity is finite. The aLIGO (LIGO Scientific Collaboration et al. 2015) detectors are sensitive to $\sim 10 \mathrm{~Hz}$, at design sensitivity, while the future space-based detector LISA (Amaro-Seoane et al. 2012) will be sensitive to much lower frequencies $\sim 1 \mathrm{mHz}$. We determine the eccentricity at a specific frequency by evolving $a_{\mathrm{o}}$ and $e_{\mathrm{o}}$, according to $\langle\mathrm{d} e / \mathrm{d} a\rangle$ (Peters 1964), up until some target value $a$ associated with the frequency in consideration.

In Fig. 19, we display the residual eccentricity of the inspiralling $\mathrm{BH}-\mathrm{BH}$ binaries, as they first enter the design-sensitivity frequency bands for aLIGO and LISA. It is apparent that for aLIGO, both

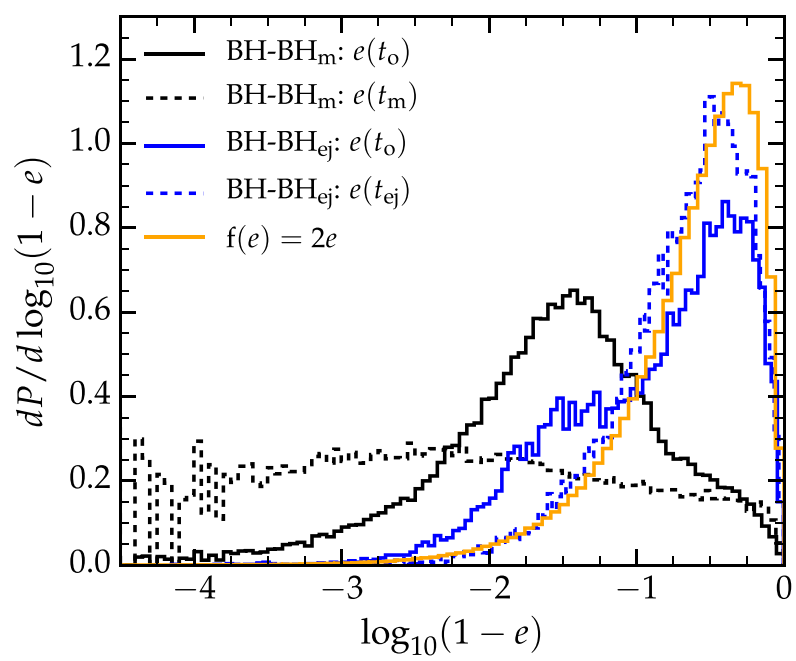

Figure 18. The probability distributions of eccentricity for two populations of $\mathrm{BH}-\mathrm{BH}$ binaries in GCs: $\mathrm{BH}-\mathrm{BH}$ binaries that form and merge in cluster ( $\mathrm{BH}-\mathrm{BH}_{\mathrm{m}}$, black lines) and the $\mathrm{BH}-\mathrm{BH}$ binaries that form and are ejected from the cluster $\left(\mathrm{BH}-\mathrm{BH}_{\mathrm{ej}}\right.$, blue lines). For each population, we show the eccentricity distribution at the time the binary forms, $e\left(t_{\mathrm{o}}\right)$ (solid lines), and the distribution of eccentricities at the binary's final state (dashed lines). The final state of the in-cluster mergers is at a time $t_{\mathrm{m}}$, the time at which the computed merger time is less than the cluster time-step. The final state for the ejected binaries is the time of ejection $t_{\mathrm{ej}}$. A thermal eccentricity distribution, with probability density $f(e)=2 e$, is included for reference.

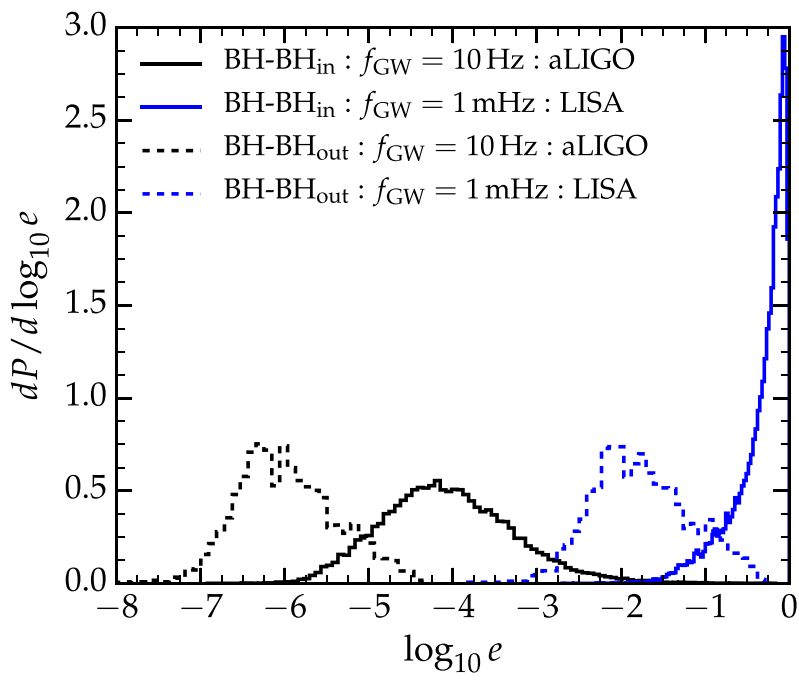

Figure 19. The eccentricity probability distributions for two populations of $\mathrm{BH}-\mathrm{BH}$ mergers from GCs for the two detectors aLIGO and LISA. The two populations correspond to the $\mathrm{BH}-\mathrm{BH}$ mergers occurring in-cluster (solid lines) and those that merge outside of the cluster, post-ejection (dashed lines). The black lines correspond to the eccentricity of each population when it reaches a corresponding gravitational wave frequency of $f_{\mathrm{GW}}=10 \mathrm{~Hz}$, the lower-bound frequency of the aLIGO band at design sensitivity. The blue lines represent the eccentricity distribution at $f_{\mathrm{GW}}=1 \mathrm{mHz}$, the proposed lower-bound frequency for LISA.

the ejected mergers and the initially high-eccentricity in-cluster mergers have residual eccentricity distributions below $10^{-1}$, which has a negligible effect on detections using circularized templates. However, in the case of LISA, while the ejected mergers result in a small eccentricity at $1 \mathrm{mHz}$, the initially highly eccentric in-cluster merger population remains significantly eccentric at this frequency. 
Table 6. The rate of three-body mergers in GCs computed for the Milky Way galaxy and stated in $\mathrm{MWEG}^{-1} \mathrm{Myr}^{-1}$. Each row corresponds to the three-body merger rate in Milky Way GCs with the simulated BH population specified by $N_{\mathrm{BH}}$ in $\left\langle R\left(N_{\mathrm{BH}}\right)\right\rangle$. The merger rates are averaged over the life of the cluster, weighted by the GC mass function to account for the nonuniform mass distribution of GCs, and assumes $N_{\mathrm{GC}} \simeq 150$ for the number of GCs in the galaxy.

\begin{tabular}{rllll}
\hline$\left\langle R\left(N_{\mathrm{BH}}\right)\right\rangle$ & $\mathrm{BH}-\mathrm{NC}$ & $\mathrm{BH}-\mathrm{WD}$ & $\mathrm{BH}-\mathrm{NS}$ & $\mathrm{BH}-\mathrm{BH}$ \\
\hline$\langle R(20)\rangle$ & $1.02 \times 10^{-1}$ & $1.60 \times 10^{-2}$ & $2.65 \times 10^{-4}$ & $1.64 \times 10^{-5}$ \\
$\langle R(200)\rangle$ & 1.08 & $1.72 \times 10^{-1}$ & $2.53 \times 10^{-3}$ & $1.40 \times 10^{-4}$ \\
$\langle R(1000)\rangle$ & 12.27 & 2.14 & $3.03 \times 10^{-2}$ & $1.11 \times 10^{-3}$ \\
\hline
\end{tabular}

Utilizing $\langle\mathrm{d} e / \mathrm{d} a\rangle$ to determine the evolved eccentricity assumes that the binary evolves in isolation. For the in-cluster mergers, we classify a BH-BH binary as merged once the orbital decay time has fallen below the cluster time-step. However, this could leave significant time for further dynamics to modify the eccentricity such that the binary will not in fact merge in cluster (Banerjee et al. 2010). To account for this possibility, the in-cluster mergers in Fig. 19 only include those mergers which satisfy the additional constraint $t_{\mathrm{dec}}<\left\langle t_{\mathrm{enc}}\right\rangle$, which is satisfied for $\sim 70$ per cent of incluster mergers. Here, the average encounter time is approximated by $\left\langle t_{\text {enc }}\right\rangle=t_{\text {bin }} / N_{\text {enc }}$ with $t_{\text {bin }}$ corresponding to the time since the binary's formation and $N_{\text {enc }}$ is the number of three-body encounters the binary has been subject to during the time $t_{\text {bin }}$. The remaining $\sim 30$ per cent of mergers are uncertain and are not further evolved; they may be broken up, ejected, or merge after subsequent interactions.

\subsubsection{Three-body mergers}

In addition to the GW-driven mergers, we also calculate the rate of tidally driven mergers or 'collisions' that occur during three-body encounters. The merger criteria are based on a minimum separation between bodies, as discussed in Section 2.3.4. We compute the expected number of three-body merger events only for those that involve a BH. Although we track the number of three-body mergers for all object types, including NS-NS, MS-WD, etc., we are missing a significant fraction of these mergers by only tracking single $\mathrm{BHs}$ or binaries with at least one $\mathrm{BH}$. We compute the expected number of mergers in a manner similar to the computation of GW mergers above.

The left columns of Table 4 list the expected number of mergers involving a $\mathrm{BH}$ that occur during three-body encounters over the lifetime of each cluster for a given $\mathrm{BH}$ population. These three-body mergers are computed using equation (45) to obtain an expected value for each cluster in the set. As the majority of these events will only be observationally relevant locally, we provide these rates solely for the Milky Way galaxy. Using the computed values from Table 4 we construct a cluster weighted average with equation (46). From this we use a modified version of equation (47), with $N_{\mathrm{GC}} \simeq$ 150 , for the approximate number of GCs in our Galaxy, in place of $\rho_{\mathrm{GC}}$ to obtain the final approximate rate for each event: $\left\langle R\left(N_{\mathrm{BH}}\right)\right\rangle=$ $\frac{\left\langle N_{\mathrm{m}}\left(N_{\mathrm{BH}}\right)\right\rangle}{t_{\mathrm{GC}}} N_{\mathrm{GC}}$. These computed rates for BH-BH, BH-NS, BH$\mathrm{WD}$, and $\mathrm{BH}-\mathrm{NC}$ are shown in Table 6 , stated in terms of the number of expected events per Milky Way equivalent galaxy (MWEG) per Myr. The BH-NC merger rate includes the three-body mergers of both BH-RG and BH-MS.

These rates are included to ensure that a large population of retained BHs in GCs does not lead to a conflict with observations. Even in the case of maximal $\mathrm{BH}$ retention, the occurrence of these events is relatively infrequent. The most commonly occurring threebody collision is that between a $\mathrm{BH}$ and a $\mathrm{NC}$ star. The interaction of a NC object with a $\mathrm{BH}$, commonly referred to as a tidal disruption event (TDE), is often studied in the context of supermassive BHs rather than stellar-mass BHs. However, there is some interest in GCrelevant $\mathrm{NC}$ collisions with stellar-mass $\mathrm{BHs}$, which are referred to as micro-TDEs (Perets et al. 2016). These events lead to full or partial tidal disruption of the NC star and are accompanied by longduration energetic flares. There is large uncertainty in the signals associated with these events as the strength and duration of the signal depends heavily on the details of the encounter (see e.g. Perets et al. 2016).

The signals associated with the compact mergers are likely to appear as head-on mergers due to the criteria associated with categorizing mergers during three-body encounters; the exclusion of higher-order corrections to Newtonian gravity in our three-body calculations requires extremely close-encounters due to the relatively small size of the compact objects involved. Despite the uncertainty in the observables produced in three-body collisions, the rate of occurrence is low enough that our model does not generate a conflict with present observations.

\subsection{Comparison with observations and previous results}

In our simulations, GCs produce a population of BH-LMXBs with a unique set of characteristic properties. These properties provide some constraints on the likelihood of a BH-LMXB having a GC origin. In this section, we identify the key characteristics of $\mathrm{BH}-$ LMXBs from GCs and determine which of the currently known $\mathrm{BH}-\mathrm{LMXBs}$ are consistent with this population.

As discussed in Section 4.2.3 and visible in Fig. 13, the spectrum of BH masses in BH-LMXBs from GCs in our simulations is roughly consistent with the observed population of $\mathrm{BH}$ masses. This makes the $\mathrm{BH}$ mass a poor candidate for differentiating between field-formed $\mathrm{BH}-\mathrm{LMXBs}$ and those with a GC origin. As a consequence of the age of GCs, the companions are typically unevolved MS stars, with masses necessarily below the turn-off mass $m_{\text {to }}=0.85 \mathrm{M}_{\odot}$. Additionally, they reside on a tightly confined branch of a temperature-luminosity diagram (see Fig. 16). This provides the first distinctive characteristic of $\mathrm{BH}-\mathrm{LMXBs}$ formed in GCs: a companion mass of $m_{2} \lesssim 0.85 \mathrm{M}_{\odot}$ and a spectral class consistent with late-type K/M stars. BlackCAT (Corral-Santana et al. 2016) currently contains 18 observed BH-LMXB systems with the proper information to compute an estimate of the companion mass. Of the $18 \mathrm{BH}-\mathrm{LMXB}$ systems, $6 \mathrm{BH}-\mathrm{LMXBs}$ have companion masses exceeding the maximum companion mass in our population of BH-LMXBs from GCs. Two of these six are near the edge of the distribution with $m_{2} \gtrsim 0.9 \mathrm{M}_{\odot}$, while the other four have $m_{2} \geq 2.52 \mathrm{M}_{\odot}$, suggesting these are more consistent with a fieldformation scenario.

A second property of a BH-LMXB with a GC origin is a characteristically short period. As shown in Fig. 15, there is a sharp limit in the distribution confining GC-origin BH-LMXBs to periods shorter than $p \sim 6.5 \mathrm{~h}$. Of the 27 confirmed BH-LMXBs with measured periods in BlackCAT, 18 have periods with $p>7 \mathrm{~h}$, indicating an unlikely GC origin for an additional set of systems. Note, however, that these systems are not necessarily distinct from those ruled unlikely on the basis of companion mass.

Although the GC-origin BH-LMXBs are more likely to reside at larger values of $|z|$ perpendicular to the Galactic plane (see Fig. 12), the overall distribution of the BH-LMXBs from GCs does not provide a strict criterion for discerning between GC origin and field 
Table 7. Properties of the five observed systems that are consistent with the properties of our simulated population of BH-LMXBs with $\mathrm{GC}$ origins. The columns refer to the primary $\mathrm{BH}$ mass $M_{\mathrm{BH}}$, the companion mass $m_{2}$, the orbital period $p$, and the absolute distance perpendicular to the Galactic plane $|z|$.

\begin{tabular}{llllll}
\hline Name & $M_{\mathrm{BH}}\left[\mathrm{M}_{\odot}\right]$ & $m_{2}\left[\mathrm{M}_{\odot}\right]$ & $p[\mathrm{~h}]$ & $|z|[\mathrm{kpc}]$ & References \\
\hline MAXI J1659-152 & $5.8 \pm 2.2$ & $0.19 \pm 0.05$ & $2.414 \pm 5 \times 10^{-3}$ & $2.45 \pm 1.05$ & {$[0,1]$} \\
SWIFT J1357.2-0933 & $>8.3$ & $>0.33$ & $2.8 \pm 3 \times 10^{-1}$ & $>1.75$ & {$[2,3]$} \\
SWIFT J1753.5-0127 & $>7.4 \pm 1.2$ & $\geq 0.30 \pm 0.03$ & $3.244 \pm 1 \times 10^{-3}$ & $1.3 \pm 0.4$ & {$[4-7]$} \\
XTE J1118+480 & $7.55 \pm 0.65$ & $0.187 \pm 0.083$ & $4.07841 \pm 1 \times 10^{-5}$ & $1.52 \pm 0.09$ & {$[8-11]$} \\
GRO J0422+32 & $8.5 \pm 6.5$ & $0.46 \pm 0.31$ & $5.09185 \pm 5 \times 10^{-6}$ & $0.51 \pm 0.06$ & {$[12-15]$} \\
\hline
\end{tabular}

References: [0] Yamaoka et al. (2012), [1] Kuulkers et al. (2013), [2] Mata Sánchez et al. (2015), [3] Corral-Santana et al. (2013), [4] Shaw et al. (2016), [5] Neustroev et al. (2014), [6] Zurita et al. (2008), [7] Cadolle Bel et al. (2007), [8] Khargharia et al. (2013), [9] Calvelo et al. (2009), [10] Torres et al. (2004), [11] Gelino et al. (2006), [12] Casares et al. (1995), [13] Beekman et al. (1997), [14] Webb et al. (2000), [15] Gelino \& Harrison (2003).

origin. Fig. 11 illustrates that while the simulated population extends much farther out of the Galactic plane than the observed distribution, there is still a significant population of GC-origin BH-LMXBs that reside in the plane, overlapping the region where field-formed binaries are expected to have the highest density. This makes discerning a potential origin for $\mathrm{BH}-\mathrm{LMXBs}$ in this region difficult. Additionally, for the many systems clustered near the Galactic centre or those that reside in the plane, the high density of objects and dust make these systems equally difficult to observe optically. Although a number of BH-LMXB candidates are detectable in these regions through X-ray, the detailed properties of these systems remain unknown due to current optical limitations. The spatial distribution of BH-LMXBs from GCs, in general, makes observations of the population difficult, even for those out of the plane. Observation and confirmation of $\mathrm{BH}-\mathrm{LMXBs}$ rely on a dynamical measurement of the BH mass through optical spectroscopy, introducing a bias towards sources at distances $D<10 \mathrm{kpc}$ from the Sun (Repetto $\&$ Nelemans 2015). For the population of BH-LMXBs from our model GCs, the MAX and 200 cases both produce a median distance of $D=9.7 \mathrm{kpc}$, placing roughly half of the systems beyond the observable range.

Although this model population has characteristics that make observations of the binary properties difficult, there are some observed systems that provide a resemblance to those with GC origins. There are 18 observed and confirmed BH-LMXBs in BlackCAT with measured quantities that allow for comparison with our simulated population. Five of the 18 systems have a BH mass, companion mass, and period consistent with the characteristics of our population of $\mathrm{BH}-$ LMXBs from GCs. These systems are MAXI J1659-152, SWIFT J1357.2-0933, SWIFT J1753.5-0127, XTE J1118+480, and GRO J0422+32. In Table 7, we list the five consistent systems and the known properties that are compatible with the range of values belonging to our population of BH-LMXBs from GCs. While we cannot make any strong claims in regards to the specific origin of these systems, it is worthwhile to note the similarities of these systems with respect to the population produced in this study.

The BH-LMXB system XTE J1118+480 is well studied, which provides some additional parameters worth comparing with our modelled population of BH-LMXBs from GCs. In addition to the consistent mass of the companion star in XTE J1118+480, the spectral type is also aligned with the band of GC-origin companions in Fig. 16. Although space-velocity measurements of BH-LMXBs are rare, fortunately there exists a velocity measurement of XTE J1118+480. In the same heliocentric Galactic coordinate system $(U, V, W)$ introduced in Section 4.2.3, Mirabel et al. (2001) found a space-velocity for this system of $(U=-105 \pm 16, V=-98 \pm 16$, $W=-21 \pm 10) \mathrm{km} \mathrm{s}^{-1}$. The large magnitude $v \sim 145 \mathrm{~km} \mathrm{~s}^{-1}$ and the large negative $V$-component are consistent with a high-velocity halo orbit and a lower than average rotational velocity about the Galactic centre. This description is consistent with the velocity distribution of our population of BH-LMXBs from GCs, which inherit the high-velocity halo-orbits when they are ejected from the GC. As a consequence of the high-velocity halo orbit, which manifests itself as a high computed peculiar velocity, this system is commonly invoked to support large natal kicks (Gualandris et al. 2005; Fragos et al. 2009; Repetto et al. 2012; Repetto \& Nelemans 2015). Confidently identifying an origin for this system could help to shed some light on the issue. The relatively low-metallicity environments of GCs provides an additional constraint on properly categorizing $\mathrm{BH}-\mathrm{LMXBs}$ as originating in GCs versus in the field. Although all of the previous characteristics point to a GC origin, perhaps one of the strongest arguments against a GC origin for this system is the supersolar abundance of elements in the secondary star found by González Hernández et al. (2006), which is consistent with a metalrich progenitor and makes a GC origin highly unlikely. However, there exist a conflicting claim presented by Frontera et al. (2001), where through broad-band $\mathrm{X}$-ray spectroscopy, it was concluded that the companion has a metallicity of $Z \sim 10^{-3}$, consistent with the low metallicities expected of systems at large $|z|$ or those with a $\mathrm{GC}$ origin. Given that metallicity provides a strong constraint on the origin of a BH-LMXB, additional observations appear necessary to reduce the uncertainty of this case.

To our knowledge, there are no known velocity measurements or metallicity measurements for the four other BH-LMXBs with possible GC origins. Although an increasing number of BH-LMXB candidates are being discovered in X-rays, only a few have been confirmed and characterized with detailed optical follow-up observations. Over time, more data will become available, better constraining the properties of the Galactic BH-LMXB population. If even a single BH-LMXB could be confidently attributed to a GC origin, this would provide a strong argument in favour of $\mathrm{BH}$ retention in GCs.

\section{DISCUSSION AND CONCLUSIONS}

There is growing observational evidence and theoretical support for a sizable BH population in present-day Galactic GCs. These BHs can acquire low-mass companions through dynamical interactions within the GC. Those binaries that are ejected from the GC can evolve into BH-LMXBs and can populate a large region of space above and below the Galactic plane. These binaries could potentially explain observed BH-LMXBs at large distances from the plane without a need for large BH birth kicks. 
In this study, we have presented a population of Milky Way BH-LMXBs formed through dynamical interactions in GCs. To explore the BH-LMXB population dependence on $\mathrm{BH}$ retention in $\mathrm{GCs}$, we performed simulations for retained $\mathrm{BH}$ populations of 20, 200, and 1000 BHs. The simulated GCs broadly cover the parameter space and represent a realistic subset of Milky Way GCs. We generated a large number of binary evolution realizations for each set of initial GC parameters and number of retained BHs. This allowed us to derive statistical distributions for the number of ejected binaries and their relevant properties. Using the statistics from the GC simulations, we performed Monte Carlo simulations to obtain a present-day population of BH-LMXBs ejected from GCs.

We find that in the case of minimal $\mathrm{BH}$ retention $\left(N_{\mathrm{BH}}=20\right)$ no observable BH-LMXBs are produced, while the $N_{\mathrm{BH}}=200$ and $N_{\mathrm{BH}}=1000$ cases yield $25_{-6}^{+10}$ and $156_{-24}^{+26} \mathrm{BH}-\mathrm{LMXBs}$, respectively. Here, the uncertainties represent the bounds of the 95 per cent confidence interval. As there is no observable population for minimal BH retention, this suggests that finding any BH-LMXB of GC origin would imply that $\mathrm{GCs}$ retain sizable $\mathrm{BH}$ populations of more than a few tens of BHs.

Aside from the difference in the size of the population, the properties and distributions of BH-LMXBs are qualitatively similar for the two cases that produce BH-LMXBs, 200 and MAX. We find that BH-LMXBs from GCs have velocity distributions inherited from their host clusters that are consistent with stars on high-velocity halo orbits. Additionally, the ejected BH-LMXBs have a spatial distribution that is also similarly aligned with the GC Galactic distribution. This shared distribution is described by a high density in the Galactic plane and near the Galactic centre, with a significant fraction distributed well above and below the Galactic plane. The typical binary is located at an absolute distance of $R=4.5 \mathrm{kpc}$ from the Galactic core when projected on to the Galactic plane, an absolute distance of $|z|=1.6 \mathrm{kpc}$ perpendicular to the Galactic plane, and at a distance of $D=9.74 \mathrm{kpc}$ from the Sun. The presence of a large population of BH-LMXBs at large distances from the plane is characteristic of BH-LMXBs from GCs, as field formed BH-LMXBs must be subject to large kicks in order to access this region. The average present-day BH-LMXB ejected from a GC is composed of a $8.25 \mathrm{M}_{\odot} \mathrm{BH}$ and a $0.22 \mathrm{M}_{\odot} \mathrm{K} / \mathrm{M}$ late-type MS star below the turn-off mass, with a characteristically short orbital period of $p=0.186 \mathrm{~h}$. These properties and their associated distributions are key observable characteristics of this predicted population of BH-LMXBs formed in GCs.

Comparing our BH-LMXB systems with the ensemble of observed $\mathrm{BH}-\mathrm{LMXBs}$, we find that five of these are candidates for having a GC origin. There are a total of 27 confirmed BH-LMXBs, but just 18 of these have sufficient observations for comparing measured properties against our results. The five systems that are compatible with our simulated population of BH-LMXBs from GCs are MAXI J1659-152, SWIFT J1357.2-0933, SWIFT J1753.5-0127, XTE J1118+480, and GRO J0422+32. XTE J1118+480 is one of the rare systems with a measured space velocity and it is atypically large for a system formed in the Galactic disc, with $v \sim 145 \mathrm{~km} \mathrm{~s}^{-1}$. This system is commonly discussed in the context of formation kicks, since a high-velocity kick is required to explain the large distance from the Galactic plane, $|z| \sim 1.52 \mathrm{kpc}$, under the assumption that it originated in the plane. However, if XTE J1118+480 comes from a GC, which produces BH-LMXBs at a median distance of $|z| \sim 1.6 \mathrm{kpc}$ from the plane, then its position and velocity are a natural consequence of the $\mathrm{GC}$ origin and do not require a large $\mathrm{BH}$ birth kick.
Future observations of the remaining four system velocities would provide an important additional piece of evidence in each of these cases. Additionally, the companion stars in BH-LMXBs from GCs should have the same low metallicity as is typical for GCs. This emphasizes the need for reliable metallicity measurements of the companion metallicity, which could help to support or reject a GC origin scenario. The strength in this measurement relies on the distinctly low-metallicity environments of GCs compared to the disc environment. The metallicity of the companion in XTE J1118+480 has been measured by Frontera et al. (2001) and González Hernández et al. (2006). However, the two measurements disagree, with the former finding sub and the latter finding super solar metallicity. Additional observations may be necessary to settle the discussion for XTE J1118+480. Future observations will be needed to more reliably determine or rule out the potential $\mathrm{GC}$ origin of the candidate BH-LMXBs. On the basis of our GC simulations, we reaffirm that if one or multiple can be shown to come from a GC, then GCs retain sizable BH populations.

An additional result from our simulations is a prediction for the $\mathrm{BH}-\mathrm{BH}$ merger rate as function of the $\mathrm{GC}-\mathrm{BH}$ population. The expected rate of mergers due to all GCs for our maximum retention case, $N_{\mathrm{BH}}=1000$, is $4.81 \mathrm{Gpc}^{-3} \mathrm{yr}^{-1}$, while in the case of minimal retention, $N_{\mathrm{BH}}=20$, the rate is as low as $3.95 \times 10^{-2} \mathrm{Gpc}^{-3} \mathrm{yr}^{-1}$. This rate represents an average over the cluster lifetimes and assumes a spatial density of GCs throughout the Universe of $\rho_{\mathrm{GC}}=0.77 \mathrm{Mpc}^{-3}$. Our maximum retention rate is consistent with previous estimates of the GC merger rate contribution and is compatible with the recent observations by aLIGO. Although our model produces rates in good agreement with previous studies, our simulations result in a larger than average fraction of mergers occurring in-cluster, as opposed to post-ejection. We attribute the discrepancy to the increased interaction between the $\mathrm{BHs}$ and the lower-mass stars as a consequence of our cluster $\mathrm{BH}$ distribution. The $\mathrm{BH}-\mathrm{BH}$ binaries that merge in-cluster are a consequence of the large eccentricities, acquired through dynamical formation, leading to significantly shortened orbital decay times. The dynamically formed $\mathrm{BH}-\mathrm{BH}$ binaries that merge in-cluster are formed with an average eccentricity of $e \sim 0.96$. At the time of merger in the aLIGO band, the residual eccentricities are small and in the range $10^{-6} \lesssim e \lesssim$ $10^{-2}$. However, we find that when passing through the LISA band years before merger, they still have eccentricities in the range $10^{-2} \lesssim$ $e \lesssim 1$. Models in which the BHs are confined to a sub-cluster at the core of GCs produce mergers with substantially smaller eccentricities. As the merger formation channels are sufficiently different for a BH sub-cluster model, LISA might be able to help distinguish how a population of retained BHs is distributed in GCs by observing the distribution of eccentricities.

The present study provides new insights into the population and properties of BH-LMXBs of GC origin. However, there are a number of important limitations that should be kept in mind when interpreting our results. While there is mounting evidence to support that present-day GCs are BH retaining, how GCs are able to retain a significant population of $\mathrm{BHs}$ and how those $\mathrm{BHs}$ are distributed is still uncertain. Our choice of distributing the BHs throughout the cluster is motivated by preserving the observed structural properties of each modelled GC in the presence of a large $\mathrm{BH}$ population. However, this spreading leads to an increase in interaction between the BHs and the lower-mass stars, which is typically a rare occurrence if the BHs remain clustered in the core. If GCs are able to retain a significant population of $\mathrm{BHs}$ that remain centrally clustered, formation of $\mathrm{BH}-\mathrm{NC}$ binaries will likely be suppressed. The reduced formation of $\mathrm{BH}-\mathrm{NC}$ binaries would significantly reduce 
the number of ejected $\mathrm{BH}-\mathrm{NCs}$, directly diminishing the number of BH-LMXBs from GCs. Future studies regarding the impact of the $\mathrm{BH}$ distribution within $\mathrm{BH}$-retaining GCs are necessary to fully understand the consequences of this limitation. Furthermore, the results presented here rely on the outcomes of many independent realizations. Since we perform each simulation independently in a static cluster background, we are neglecting the change in the $\mathrm{BH}$ population and its impact on the cluster as single $\mathrm{BHs}$ and $\mathrm{BH}$ binaries are ejected over the cluster lifetime. Additionally, we do not account for binary-binary interactions, which have the potential to disrupt existing binaries or possibly aid in ejecting them. Models that account for these limitations are necessary to better understand the impact of ignoring these processes. While $N$-body simulations and Monte-Carlo-based models can resolve some of these issues, the computational expense remains a limiting factor in performing many realizations. However, as the computational techniques and resources continue to improve, it will soon be possible to produce many high-accuracy GC simulations that address these limitations.

\section{ACKNOWLEDGEMENTS}

The authors thank Sterl Phinney, Steinn Sigurdsson, and Saul Teukolsky for valuable discussions. Additionally, we thank the anonymous referee for helpful comments on this manuscript. This work is partially supported by the Sherman Fairchild Foundation and by NSF under award no. CAREER PHY-1151197. The simulations were carried out on NSF/NCSA Blue Waters under PRAC award no. ACI-1440083 and on the Caltech cluster Zwicky, supported by the Sherman Fairchild Foundation and NSF award no. PHY-0960291.

\section{REFERENCES}

Aarseth S. J., 2012, MNRAS, 422, 841

Abbott B. P. et al., 2016a, Phys. Rev. Lett., 116, 061102

Abbott B. P. et al., 2016b, Phys. Rev. Lett., 116, 241103

Abbott B. P. et al., 2016c, ApJ, 818, L22

Abbott B. P. et al., 2016d, ApJ, 818, L22

Abbott B. P. et al., 2017, Phys. Rev. Lett., 118, 221101

Amaro-Seoane P. et al., 2012, Class. Quantum Gravity, 29, 124016

Bahcall N. A., Hausman M. A., 1977, ApJ, 213, 93

Banerjee S., Baumgardt H., Kroupa P., 2010, MNRAS, 402, 371

Barnard R., Garcia M., Li Z., Primini F., Murray S. S., 2011, ApJ, 734, 79

Beekman G., Shahbaz T., Naylor T., Charles P. A., Wagner R. M., Martini P., 1997, MNRAS, 290, 303

Belczynski K., Sadowski A., Rasio F. A., Bulik T., 2006, ApJ, 650, 303 Benacquista M. J., Downing J. M. B., 2013, Living Rev. Relativ., 16, 4 Benz W., Hills J. G., 1992, ApJ, 389, 546

Binney J., Tremaine S., 2008, Galactic Dynamics, 2nd edn. Princeton Univ. Press, Princeton, NJ

Blaauw A., 1961, Bull. Astron. Inst. Netherlands, 15, 265

Bovy J., 2015, ApJS, 216, 29

Breen P. G., Heggie D. C., 2013, MNRAS, 432, 2779

Cadolle Bel M. et al., 2007, ApJ, 659, 549

Calvelo D. E., Vrtilek S. D., Steeghs D., Torres M. A. P., Neilsen J., Filippenko A. V., González Hernández J. I., 2009, MNRAS, 399, 539

Campanelli M., Lousto C., Zlochower Y., Merritt D., 2007, ApJ, 659, L5

Casares J., Jonker P. G., 2014, Space Sci. Rev., 183, 223

Casares J., Martin A. C., Charles P. A., Martin E. L., Rebolo R., Harlaftis E. T., Castro-Tirado A. J., 1995, MNRAS, 276, L35

Catalán S., Isern J., García-Berro E., Ribas I., 2008, MNRAS, 387, 1693

Chernoff D. F., Weinberg M. D., 1990, ApJ, 351, 121

Clausen D., Wade R. A., Kopparapu R. K., O’Shaughnessy R., 2012, ApJ, 746,186
Clausen D., Sigurdsson S., Chernoff D. F., 2013, MNRAS, 428, 3618

Cohn H., 1979, ApJ, 234, 1036

Corral-Santana J. M., Casares J., Muñoz-Darias T., Rodríguez-Gil P., Shahbaz T., Torres M. A. P., Zurita C., Tyndall A. A., 2013, Science, 339, 1048

Corral-Santana J. M., Casares J., Muñoz-Darias T., Bauer F. E., MartínezPais I. G., Russell D. M., 2016, A\&A, 587, A61

Davies M. B., 1995, MNRAS, 276, 887

Davies M. B., Benz W., 1995, MNRAS, 276, 876

Davies M. B., Benz W., Hills J. G., 1994, ApJ, 424, 870

de Mink S. E., Sana H., Langer N., Izzard R. G., Schneider F. R. N., 2014 ApJ, 782, 7

Di Stefano R., Kong A. K. H., Garcia M. R., Barmby P., Greiner J., Murray S. S., Primini F. A., 2002, ApJ, 570, 618

Downing J. M. B., Benacquista M. J., Giersz M., Spurzem R., 2011, MNRAS, 416,133

Duric N., 2004, Advanced Astrophysics. Cambridge Univ. Press, New York Fragos T., Willems B., Kalogera V., Ivanova N., Rockefeller G., Fryer C. L., Young P. A., 2009, ApJ, 697, 1057

Fregeau J. M., Ivanova N., Rasio F. A., 2009, ApJ, 707, 1533

Frontera F. et al., 2001, ApJ, 561, 1006

Gelino D. M., Harrison T. E., 2003, ApJ, 599, 1254

Gelino D. M., Balman Ş., Kızıloğlu Ü., Yılmaz A., Kalemci E., Tomsick J. A., 2006, ApJ, 642, 438

González Hernández J. I., Rebolo R., Israelian G., Harlaftis E. T., Filippenko A. V., Chornock R., 2006, ApJ, 644, L49

Gualandris A., Colpi M., Portegies Zwart S., Possenti A., 2005, ApJ, 618, 845

Harris W. E., 1996, AJ, 112, 1487

Heggie D. C., Hut P., McMillan S. L. W., 1996, ApJ, 467, 359

Hénon M. H., 1971, Ap\&SS, 14, 151

Hurley J. R., Tout C. A., Pols O. R., 2002, MNRAS, 329, 897

Hut P., Bahcall J. N., 1983, ApJ, 268, 319

Hut P., McMillan S., Romani R. W., 1992, ApJ, 389, 527

Ivanova N., Belczynski K., Fregeau J. M., Rasio F. A., 2005, MNRAS, 358 , 572

Ivanova N., Heinke C. O., Rasio F. A., Belczynski K., Fregeau J. M., 2008, MNRAS, 386, 553

Ivanova N., Chaichenets S., Fregeau J., Heinke C. O., Lombardi J. C., Jr, Woods T. E., 2010, ApJ, 717, 948

Ivanova N., da Rocha C. A., Van K. X., Nandez J. L. A., 2017, ApJ, 843 , L30

Janka H.-T., 2013, MNRAS, 434, 1355

Janka H.-T., 2017, ApJ, 837, 84

Jeans J. H., 1919, MNRAS, 79, 408

Jonker P. G., Nelemans G., 2004, MNRAS, 354, 355

Kharchenko N. V., Piskunov A. E., Schilbach E., Röser S., Scholz R.-D., 2013, A\&A, 558, A53

Khargharia J., Froning C. S., Robinson E. L., Gelino D. M., 2013, AJ, 145, 21

King I. R., 1966, AJ, 71, 64

Krauss L. M., Chaboyer B., 2003, Science, 299, 65

Kroupa P., 2001, MNRAS, 322, 231

Kulkarni S. R., Hut P., McMillan S., 1993, Nature, 364, 421

Kuulkers E. et al., 2013, A\&A, 552, A32

Lamberts A., Garrison-Kimmel S., Clausen D. R., Hopkins P. F., 2016, MNRAS, 463, L31

Lee H. M., Ostriker J. P., 1986, ApJ, 310, 176

LIGO Scientific Collaboration et al., 2015, Class. Quantum Grav., 32, 074001

Lyne A. G., Lorimer D. R., 1994, Nature, 369, 127

Mandel I., 2016, MNRAS, 456, 578

Mata Sánchez D., Muñoz-Darias T., Casares J., Corral-Santana J. M., Shahbaz T., 2015, MNRAS, 454, 2199

McClintock J. E., Remillard R. A., 2006, in Lewin W., van der Klis M., eds, Black Hole Binaries In: Compact stellar X-ray sources, Cambridge Astrophysics Series 39. Cambridge Univ. Press, Cambridge, p. 157

McLaughlin D. E., 2000, ApJ, 539, 618 
McLaughlin D. E., Pudritz R. E., 1996, ApJ, 457, 578

Meylan G., Heggie D. C., 1997, A\&AR, 8, 1

Milone A. P. et al., 2012, A\&A, 540, A16

Mirabel I. F., Dhawan V., Mignani R. P., Rodrigues I., Guglielmetti F., 2001, Nature, 413, 139

Moreno E., Pichardo B., Velázquez H., 2014, ApJ, 793, 110

Morozova V., Piro A. L., Renzo M., Ott C. D., Clausen D., Couch S. M., Ellis J., Roberts L. F., 2015, ApJ, 814, 63

Morscher M., Umbreit S., Farr W. M., Rasio F. A., 2013, ApJ, 763, L15

Morscher M., Pattabiraman B., Rodriguez C., Rasio F. A., Umbreit S., 2015, ApJ, 800, 9

Neustroev V. V., Veledina A., Poutanen J., Zharikov S. V., Tsygankov S. S., Sjoberg G., Kajava J. J. E., 2014, MNRAS, 445, 2424

O’Leary R. M., Rasio F. A., Fregeau J. M., Ivanova N., O'Shaughnessy R., 2006, ApJ, 637, 937

Özel F., Psaltis D., Narayan R., McClintock J. E., 2010, ApJ, 725, 1918

Paxton B., Bildsten L., Dotter A., Herwig F., Lesaffre P., Timmes F., 2011, ApJS, 192, 3

Perets H. B., Li Z., Lombardi J. C., Jr, Milcarek S. R., Jr, 2016, ApJ, 823, 113

Peters P. C., 1964, Phys. Rev., 136, 1224

Peterson C. J., King I. R., 1975, AJ, 80, 427

Pfahl E., Rappaport S., Podsiadlowski P., 2002, ApJ, 573, 283

Repetto S., Nelemans G., 2015, MNRAS, 453, 3341

Repetto S., Davies M. B., Sigurdsson S., 2012, MNRAS, 425, 2799

Rodriguez C. L., Morscher M., Pattabiraman B., Chatterjee S., Haster C.-J., Rasio F. A., 2015, Phys. Rev. Lett., 115, 051101

Rodriguez C. L., Chatterjee S., Rasio F. A., 2016a, Phys. Rev. D, 93, 084029

Rodriguez C. L., Morscher M., Wang L., Chatterjee S., Rasio F. A., Spurzem R., 2016b, MNRAS, 463, 2109
Sadowski A., Belczynski K., Bulik T., Ivanova N., Rasio F. A., O’Shaughnessy R., 2008, ApJ, 676, 1162

Salpeter E. E., 1955, ApJ, 121, 161

Sana H. et al., 2012, Science, 337, 444

Shaw A. W., Charles P. A., Casares J., Hernández Santisteban J. V., 2016, MNRAS, 463, 1314

Sigurdsson S., Hernquist L., 1993, Nature, 364, 423

Sigurdsson S., Phinney E. S., 1993, ApJ, 415, 631

Sigurdsson S., Phinney E. S., 1995, ApJS, 99, 609

Sippel A. C., Hurley J. R., 2013, MNRAS, 430, L30

Spitzer L., 1987, Dynamical Evolution of Globular Clusters. Princeton Univ. Press, Princeton, NJ

Spitzer L., Hart M. H., Jr, 1971, ApJ, 164, 399

Strader J., Chomiuk L., Maccarone T. J., Miller-Jones J. C. A., Seth A. C., 2012, Nature, 490, 71

Torres M. A. P., Callanan P. J., Garcia M. R., Zhao P., Laycock S., Kong A. K. H., 2004, ApJ, 612, 1026

Wang C., Jia K., Li X.-D., 2016, MNRAS, 457, 1015

Webb N. A., Naylor T., Ioannou Z., Charles P. A., Shahbaz T., 2000, MNRAS, 317, 528

Yamaoka K. et al., 2012, PASJ, 64, 32

Zonoozi A. H., Küpper A. H. W., Baumgardt H., Haghi H., Kroupa P., Hilker M., 2011, MNRAS, 411, 1989

Zurita C., Durant M., Torres M. A. P., Shahbaz T., Casares J., Steeghs D., 2008, ApJ, 681, 1458

This paper has been typeset from a $\mathrm{T}_{\mathrm{E} X / \mathrm{LT} \mathrm{T}} \mathrm{X}$ file prepared by the author. 